NBER WORKING PAPER SERIES

DEALING WITH THE TRILEMMA:

OPTIMAL CAPITAL CONTROLS WITH FIXED EXCHANGE RATES

\author{
Emmanuel Farhi \\ Ivan Werning \\ Working Paper 18199 \\ http://www.nber.org/papers/w18199 \\ NATIONAL BUREAU OF ECONOMIC RESEARCH \\ 1050 Massachusetts Avenue \\ Cambridge, MA 02138 \\ June 2012
}

For useful comments and suggestions we thank Olivier Blanchard and Ricardo Reis. Adrien Auclert and Nicolas Caramp provided excellent research assistance. The views expressed herein are those of the authors and do not necessarily reflect the views of the National Bureau of Economic Research.

NBER working papers are circulated for discussion and comment purposes. They have not been peerreviewed or been subject to the review by the NBER Board of Directors that accompanies official NBER publications.

(C) 2012 by Emmanuel Farhi and Ivan Werning. All rights reserved. Short sections of text, not to exceed two paragraphs, may be quoted without explicit permission provided that full credit, including $\odot$ notice, is given to the source. 
Dealing with the Trilemma: Optimal Capital Controls with Fixed Exchange Rates

Emmanuel Farhi and Ivan Werning

NBER Working Paper No. 18199

June 2012

JEL No. E5,F3,F32,F33,F41,F42

\begin{abstract}
We lay down a standard macroeconomic model of a small open economy with a fixed exchange rate and study optimal capital controls (defined as maximizing the utility of a representative household). We provide sharp analytical and numerical characterizations for a variety of shocks. We find that capital controls are employed to respond to some shocks but not others. They are particularly effective to address risk-premium shocks that affect the interest rate differential foreign investors require in a particular country. We also discuss how the solution depends on the degree of nominal rigidity and the openness of the economy. We show that capital controls may be optimal even if the exchange rate is not fixed in response to risk premium shocks or if wages, in addition to prices, are sticky. Finally, we compare the single country's optimum to a coordinated world solution. Our results show a limited need for coordination. However, the uncoordinated solution features the same capital controls as the coordinated solution.
\end{abstract}

Emmanuel Farhi

Harvard University

Department of Economics

Littauer Center

Cambridge, MA 02138

and NBER

efarhi@harvard.edu

Ivan Werning

Department of Economics

MIT

50 Memorial Drive, E51-251a

Cambridge, MA 02142-1347

and NBER

iwerning@mit.edu 


\title{
Dealing with the Trilemma: Optimal Capital Controls with Fixed Exchange Rates*
}

\author{
Emmanuel Farhi \\ Harvard University
}

\author{
Iván Werning \\ MIT
}

June 2012

\begin{abstract}
We lay down a standard macroeconomic model of a small open economy with a fixed exchange rate and study optimal capital controls (defined as maximizing the utility of a representative household). We provide sharp analytical and numerical characterizations for a variety of shocks. We find that capital controls are employed to respond to some shocks but not others. They are particularly effective to address risk-premium shocks that affect the interest rate differential foreign investors require in a particular country. We also discuss how the solution depends on the degree of nominal rigidity and the openness of the economy. We show that capital controls may be optimal even if the exchange rate is not fixed in response to risk premium shocks or if wages, in addition to prices, are sticky. Finally, we compare the single country's optimum to a coordinated world solution. Our results show a limited need for coordination. However, the uncoordinated solution features the same capital controls as the coordinated solution.
\end{abstract}

\section{Introduction}

Capital flows have been extensively blamed for episodes of booms and busts in emerging markets (Calvo, 1998). They may also have contributed to the ongoing crisis in the eurozone-capital inflows and real appreciations in the periphery during the boom, as well as capital flight during the downturn. These concerns are especially relevant when the exchange rate is fixed, as it was for a number of emerging markets seeking to stabilize inflation and more recently in the EMU. Indeed, Mundell's trilemma states that a country cannot simultaneously have free capital flows, independent monetary policy, and a fixed exchange rate. How then should countries that have fixed their exchange rate cope

${ }^{*}$ For useful comments and suggestions we thank Olivier Blanchard and Ricardo Reis. Adrien Auclert and Nicolas Caramp provided excellent research assistance. 
with macroeconomic shocks? To what degree should they give up on free capital mobility to regain monetary policy? Although the International Monetary Fund has recently sided more sympathetically with the use of capital controls (Ostry et al., 2010), we still lack a benchmark model to answer these macro-stabilization questions. Our goal is to fill this gap by studying optimal (defined as maximizing the utility of the representative household) capital control policy in a standard open economy model with fixed exchange rates.

Our model, which builds on Clarida, Gali and Gertler (2002) and Galí and Monacelli $(2005,2008)$, introduces capital controls in an open economy model with nominal rigidities. We study the optimal use of capital controls in response to a great variety of shocks, including the usual productivity shocks, as well as fluctuations in export demand, terms of trade, foreign interest rates and risk premia. The latter shocks are especially relevant for us because they are a proximate cause of capital movements. Risk premium shocks have been suggested as important drivers of the business cycle in emerging open economies (see Neumeyer and Perri, 2005). We analyze different price setting assumptions: flexible prices, rigid prices, one-period in advance sticky prices, and Calvo price setting. As we show, the optimal use of capital controls depends crucially on the nature of the shock, on the stickiness of prices, and on the openness of the economy. Capital controls are more effective the more closed the economy and they are particularly powerful to respond to fluctuations in the risk premium demanded by foreign investors. Finally, we contrast the single country optimum, taking as given the rest of the world, with a cooperative world optimum. Although there are gains from coordination, we show that they take an unexpected and somewhat limited form.

We start with the case of flexible prices. Even with flexible prices, optimal capital controls are generally nonzero, a point explored in detail in Costinot et al. (2011). ${ }^{1}$ In our context, with a small open economy there is no ability to affect the world interest rate. However, each country still has some monopoly power over their terms of trade. Without trade barriers capital controls emerge as an imperfect tool to manipulate terms of trade. By reallocating spending intertemporally a country can raise their export prices in some periods and lower them in others. This effect is not the focus of this paper. Indeed, we isolate a few cases where this effect is not at play: when shocks are permanent, or in the Cole-Obstfeld parametrization (unitary inter- and intra-temporal elasticities). Nevertheless, flexible prices act as a benchmark to compare our results with nominal rigidities.

\footnotetext{
${ }^{1}$ Jeanne (2011) shows how capital controls can be used to affect the real exchange rate in a model with non-tradable goods, where terms of trade are fixed internationally.
} 
We first contrast the case of flexible prices with its polar opposite: perfectly rigid prices. Thus, we assume that the exchange rate and prices are fixed forever. As with flexible prices, capital controls are not employed in response to permanent shocks. In response to transitory shocks, however, capital controls now play a more important role. They are used to react to all the shocks we consider. A useful example is the Cole-Obstfeld case with a trend in productivity. In this case, optimal capital controls are zero when prices are flexible, but they take the form of a tax on inflows (or a subsidy on outflows) when prices are completely rigid. With flexible prices, the country's price index decreases over time. This expected deflation raises the real interest rate and increases the growth rate of consumption. With rigid prices, the real interest rate is fixed and hence the growth rate of consumption is too low, inducing a boom relative to the flexible price allocation. By taxing inflows (or subsidizing outflows) the country can increase its nominal interest rate, cooling off the economy. The growth rate of output is also increased, but by less, moving the trade balance into surplus. In contrast, with flexible prices trade is always balanced in the Cole-Obstfeld case. This underscores that capital controls are a second best tool, allowing the country to regain some monetary autonomy and therefore some control over the intertemporal allocation of spending.

We then study the same model under the intermediate assumption that prices are set one period in advance, just as in Obstfeld and Rogoff (1995). In this case capital controls have some subtle effects. As explained above, capital controls lead to intertemporal wealth transfers. Wealth transfers, in turn, lead to terms of trade and real exchange rate changes-familiar at least Keynes (1929) and Ohlin (1929). Taking these effects into account complicates the analysis, yet we are able to provide tight characterizations for the use of capital controls in this context, both for transitory and for permanent shocks of different kinds. In contrast to the case with rigid prices, capital controls are now also employed in response to permanent shocks. Indeed, the responses are qualitatively similar to those for transitory shocks. Intuitively, when prices eventually adjust, even permanent shocks induce transitory movements in economic variables, which capital controls can help stabilize to improve welfare.

We then turn to the case with staggered Calvo pricing, the workhorse assumption in New Keynesian models. In addition to allowing for more realistic dynamics, this has two important implications for our analysis. First, it introduces a welfare cost from inflationabsent in all previous cases. Second, because capital controls affect the dynamics of inflation, it creates a prudential motive for policy. Forward looking policy will consider the effects that current and future temporary shocks have on absolute and relative prices. With sticky prices these changes may have undesirable consequences in other periods. 
For example, a temporary capital inflow may heat up the economy and appreciate the real exchange rate in a way that is harmful once these flows are reverted.

We provide closed-form solutions for some revealing cases and also explore the model numerically. As was the case with prices set one period in advance, capital controls are employed in response to permanent shock, not just temporary shocks. However, now that prices adjust continuously, the response of capital controls is also more drawn out. In response to variations in risk premia, capital controls mitigate variations in the domestic nominal interest rate, but do not perfectly stabilize it. In the closed economy limit it does; capital controls vary one for one with the risk premium. Our numerical simulations show that capital controls are employed counter-cyclically for all shocks and are quite effective, especially in response to risk premium shocks. We also find that capital controls slow down inflationary dynamics and the adjustment of relative prices. In this way, they help stabilize the real exchange rate.

We also provide some new results regarding the role of openness, captured in our model by a home-bias preference parameter. One may imagine that the constraints imposed by a fixed exchange diminish if the economy is less open and perhaps vanish in the closed economy limit. Without capital controls we show that this limit result is not true. This lack of continuity with the closed economy model does not rely on the fact that a fixed exchange rate constrains the interest rate response. It holds even for shocks that do not affect the natural rate of interest, e.g. a perfectly permanent shock to productivity. However, with capital controls perfect stabilization does obtain in some, but not all, cases. In particular, it holds when prices are set one period in advance or, in the Calvo case, in response to risk premium shocks. But note that, even with optimal capital controls, the economy cannot be perfectly stabilized in response to other shocks, e.g. shocks to productivity.

Flexible exchange rates are not always perfect. We highlight two situations where capital controls are used even with a flexible exchange rate. The first case is that of a risk premium shock. The second case is the case where wages, in addition to prices, are sticky. In first case, variations in risk premia create capital flows and optimal policy leans against these to stabilize the current account and stabilize the nominal exchange rate. Thus, a lower premium is met with taxes on capital inflows to moderate both capital inflows and the nominal exchange rate appreciation.

Returning to the case with fixed exchange rates, we also study government spending as an alternative stabilization tool. Interestingly, although countercyclical spending is generally optimal, in contrast with capital controls, their use is independent of the degree of openness. Moreover, following Werning (2012) we show that in some cases op- 
timal government spending can be chosen by considering the opportunistic motive for spending alone, in other words, considering only the fact that public goods are cheaper to produce in a downturn, without paying attention to its stabilization or stimulus effects.

Up to this point we have discussed the problem of a small open economy with a fixed exchange rate, taking as given conditions in the rest of the world, including policy choices by other countries, equilibrium interest rates and prices. We also consider whether capital controls have beggar-thy-neighbor effects and whether there are gains from coordinating their use. We contrast the coordinated solution within a monetary union where countries cooperate on capital controls to maximize the sum of utilities, to the uncoordinated, noncooperative equilibrium, where each country acts in isolation. Perhaps surprisingly, we find a rather limited role for coordination. In particular, although the aggregates may or may not differ, capital controls imposed by each country are identical at the uncoordinated equilibrium and coordinated optimum. Interestingly, the gains from coordination are independent of the degree of heterogeneity across countries.

The international monetary literature is vast and has led to a detailed understanding of how monetary policy should be used in open economies (for a recent review see Corsetti et al. 2010). In comparison, stabilization policy when monetary policy is constrained by fixed exchange rates, as in a currency union, remains largely unexplored. In their classical treatments, Mundell (1963) and Fleming (1962) suggested that with fixed exchange rates and perfect capital mobility one must give up monetary policy, which may be detrimental to macroeconomic stabilization. Can capital controls then offer a partial substitute to monetary policy in such a situation? If so how should they be used and how effective are they? The main contribution of our paper is to provide an extensive analysis of these questions in a standard open economy model. Our goal is to offer a useful counterpart to existing analyses of monetary policy. In this vein, we provide sharp results characterizing the optimal use and effectiveness of capital controls in reaction to a large variety shocks. We emphasize the roles played by the persistence of disturbances, the degree of nominal rigidity and the openness of the economy. Taken together, our results help extend our understanding of stabilization policy past situations where the exchange rate is flexible.

In recent work, Schmitt-Grohe and Uribe (2012) study a disequilibrium model featuring downward rigid wage and a fixed exchange rate. Although our models are quite different, their reported simulations show that capital controls are used counter cyclically, consistent with our results on the optimal use of capital controls. A large literature in international macroeconomics is motivated by the volatility of capital flows, especially "sudden stops", see Mendoza (2010) and the references therein. Models with financial frictions such as Caballero and Krishnamurthy $(2001,2004)$ emphasize domestic and in- 
ternational collateral constraints that create inefficiencies and a potential role for intervention in international borrowing, even without nominal rigidities. A related strand of work emphasizes pecuniary externalities that work through prices in borrowing constraints, for example Bianchi and Mendoza (2010), Bianchi (2011), Jeanne and Korinek (2010), Korinek (2011). All these papers provide a rationale for "prudential" policies that attempt to prevent excessive borrowing. Martin and Taddei (2010) provide a different mechanism, with adverse selection, by which international lending is inefficient.

\section{A Small Open Economy}

We build on the framework by Galí and Monacelli $(2005,2008)$, who develop a model composed of a continuum of open economies. Our main focus is on policy in a single country, which we call Home, taking as given the rest of the world, which we call Foreign. However, we also explore the joint policy problem for the entire world when coordination is possible. We make a few departures from Gali and Monacelli's model to adapt it to the questions we address. First and foremost, they studied monetary and fiscal policy, so we must extend the model to include capital controls. Second, in contrast to their simplifying assumption of complete markets, we prefer to assume international financial markets are incomplete. No risk sharing between countries is allowed, only risk free borrowing and lending. Given this assumption, to keep the analysis tractable, we limit our attention to one-time unanticipated shocks to the economy. ${ }^{2}$ Third, Gali and Monacelli confine their normative analysis to the Cole-Obstfeld parameter specification, where various elasticities of substitution are unity. This case is more tractable and we will make extensive use of this fact here too. However, we also include some results outside of this parameter configuration. Finally, we also consider a larger variety of shocks to the economy and consider extensions where we include wage rigidity alongside price stickiness.

\subsection{Households}

There is a continuum measure one of countries $i \in[0,1]$. We focus attention on a single country, which we call Home, and can be thought of as a particular value $H \in[0,1]$. In every country, there is a representative household with preferences represented by the

\footnotetext{
${ }^{2}$ Relative to the literature, this is not a limitation since most studies, including Gali-Monacelli, work with linearized equilibrium conditions, so that the response to shocks is unaffected by the presence of future shocks.
} 
utility function

$$
\sum_{t=0}^{\infty} \beta^{t}\left[\frac{C_{t}^{1-\sigma}}{1-\sigma}-\frac{N_{t}^{1+\phi}}{1+\phi}\right],
$$

where $N_{t}$ is labor, and $C_{t}$ is a consumption index defined by

$$
C_{t}=\left[(1-\alpha)^{\frac{1}{\eta}} C_{H, t}^{\frac{\eta-1}{\eta}}+\alpha^{\frac{1}{\eta}} C_{F, t}^{\frac{\eta-1}{\eta}}\right]^{\frac{\eta}{\eta-1}},
$$

where $C_{H, t}$ is an index of consumption of domestic goods given by

$$
C_{H, t}=\left(\int_{0}^{1} C_{H, t}(j)^{\frac{\epsilon-1}{\epsilon}} d j\right)^{\frac{\epsilon}{\epsilon-1}},
$$

where $j \in[0,1]$ denotes an individual good variety. Similarly, $C_{F, t}$ is a consumption index of imported goods given by

$$
C_{F, t}=\left(\int_{0}^{1} \Lambda_{i, t}^{\frac{1}{\gamma}} C_{i, t}^{\frac{\gamma-1}{\gamma}} d i\right)^{\frac{\gamma}{\gamma-1}},
$$

where $C_{i, t}$ is, in turn, an index of the consumption of varieties of goods imported from country $i$, given by

$$
C_{i, t}=\left(\int_{0}^{1} C_{i, t}(j)^{\frac{\epsilon-1}{\epsilon}} d j\right)^{\frac{\epsilon}{\epsilon-1}} .
$$

Thus, $\epsilon$ is the elasticity between varieties produced within a given country, $\eta$ the elasticity between domestic and foreign goods, and $\gamma$ the elasticity between goods produced in different foreign countries. An important special case obtains when $\sigma=\eta=\gamma=1$. We call this the Cole-Obstfeld case, in reference to Cole and Obstfeld (1991). This case is more tractable and has some special implications that are worth highlighting. Thus, we devote special attention to it, although we will also derive results away from it.

The parameter $\alpha$ indexes the degree of home bias, and can be interpreted as a measure of openness. Consider both extremes: as $\alpha \rightarrow 0$ the share of foreign goods vanishes; as $\alpha \rightarrow 1$ the share of home goods vanishes. Since the country is infinitesimal, the latter captures a very open economy without home bias; the former a closed economy barely trading with the outside world.

We have included a taste shifter $\Lambda_{i, t}$, which is always normalized so that $\int \Lambda_{i, t} d i=$ 1 , that affects the utility of imports from country $i$. All countries experience the same taste configuration $\left\{\Lambda_{i, t}\right\}$. Thus, variations in $\Lambda_{H, t}$ allow us to consider variations in the 
demand for Home's exports.

Households seek to maximize their utility subject to the sequence of budget constraints

$$
\begin{aligned}
\int_{0}^{1} P_{H, t}(j) C_{H, t}(j) d j & +\int_{0}^{1} \int_{0}^{1} P_{i, t}(j) C_{i, t}(j) d j d i+D_{t+1}+\int_{0}^{1} E_{i, t} D_{t+1}^{i} d i \\
& \leq W_{t} N_{t}+\Pi_{t}+T_{t}+\left(1+i_{t-1}\right) D_{t}+\int_{0}^{1} \frac{1+\tau_{t-1}}{1+\tau_{t-1}^{i}} E_{i, t}\left(1+i_{t-1}^{i}\right) D_{t}^{i} d i
\end{aligned}
$$

for $t=0,1,2, \ldots$ In this inequality, $P_{H, t}(j)$ is the price of domestic variety $j, P_{i, t}$ is the price of variety $j$ imported from country $i, W_{t}$ is the nominal wage, $\Pi_{t}$ represents nominal profits and $T_{t}$ is a nominal lump sum transfer. All these variables are expressed in domestic currency. The portfolio of home agents is composed of home and foreign bond holding: $D_{t}$ is home bond holdings of home agents, $D_{t}^{i}$ is bond holdings of country $i$ of home agents. The returns on these bonds are determined by the nominal interest rate in the home country $i_{t}$, the nominal interest rate $i_{t}^{i}$ in country $i$, and the evolution of the nominal exchange rate $E_{i, t}$ between home and country $i$. Capital controls are modeled as follows: $\tau_{t}$ is a tax on capital inflows and subsidy on capital outflows in the home country, and similarly $\tau_{t}^{i}$ is a tax on capital inflows and subsidy on capital outflows in country $i$. The proceeds of these taxes are rebated lump sum to the households at Home and country $i$, respectively. The Home country taxes inflows to make the after tax (net of any subsidy paid by their own country of origin) return to foreign investors $\left(1+i_{t-1}\right) /\left(1+\tau_{t-1}\right)$ in domestic currency.

We can re-express the household budget constraint as

$$
\begin{aligned}
P_{t} C_{t}+D_{t+1}+\int_{0}^{1} E_{i, t} D_{t+1}^{i} d i & \\
& \leq W_{t} N_{t}+\Pi_{t}+T_{t}+\left(1+i_{t-1}\right) D_{t}+\int_{0}^{1} \frac{1+\tau_{t-1}}{1+\tau_{t-1}^{i}} E_{i, t}\left(1+i_{t-1}^{i}\right) D_{t}^{i} d i,
\end{aligned}
$$


using price indices

$$
\begin{aligned}
P_{t} & =\left[(1-\alpha) P_{H, t}^{1-\eta}+\alpha P_{F, t}^{1-\eta}\right]^{\frac{1}{1-\eta}}, \\
P_{H, t} & =\left(\int_{0}^{1} P_{H, t}(j)^{1-\epsilon} d j\right)^{\frac{1}{1-\epsilon}}, \\
P_{F, t} & =\left(\int_{0}^{1} \Lambda_{i, t} P_{i, t}^{1-\gamma} d i\right)^{\frac{1}{1-\gamma}}, \\
P_{i, t} & =\left(\int_{0}^{1} P_{i, t}(j)^{1-\epsilon} d j\right)^{\frac{1}{1-\epsilon}} .
\end{aligned}
$$

Here $P_{t}$ is the Home consumer price index (CPI), $P_{H, t}$ is the Home producer price index (PPI), $P_{F, t}$ is a price index of imported goods at Home, while $P_{i, t}$ is country $i$ 's PPI.

\subsection{Firms}

Technology. A typical firm in the home economy produces a differentiated good with a linear technology given by

$$
Y_{t}(j)=A_{H, t} N_{t}(j)
$$

where $A_{H, t}$ is productivity in the home country. We denote productivity in country $i$ by $A_{i, t}$.

We allow for a constant employment tax $1+\tau^{L}$, so that real marginal cost deflated by Home PPI is given by

$$
M C_{t}=\frac{1+\tau^{L}}{A_{H, t}} \frac{W_{t}}{P_{H, t}} .
$$

We take this employment tax to be constant in our model. We explain below how it is determined.

Price-setting assumptions. We will consider a variety of price setting assumptions: flexible prices, one-period in advance sticky prices, and sticky prices a la Calvo.

As in Galí and Monacelli (2005), we maintain the assumption that the Law of One Price (LOP) holds so that at all times, the price of a given variety in different countries is identical once expressed in the same currency. This assumption is sometimes known as Producer Currency Pricing (PCP). This is sometimes contrasted with the assumption of Local Currency Pricing (LCP), where each variety's price is set separately for each country and quoted (and potentially sticky) in that country's local currency. Thus, LOP does not 
necessarily hold. It has been shown by Devereux and Engel (2003) that LCP and PCP may have different implications for monetary policy. However, for our purposes, these two polar cases are equivalent since, for the most part, we will study the model assuming fixed exchange rates.

First, consider the case of flexible prices. Firm $j$ optimally sets its price $P_{H, t}(j)$ to maximize

$$
\max _{P_{H, t}(j)}\left(P_{H, t}(j) Y_{t \mid t}-P_{H, t} M C_{t} Y_{t \mid t}\right)
$$

where $Y_{t \mid t}=\left(\frac{P_{H, t}(j)}{P_{H, t}}\right)^{-\epsilon} Y_{t}$, taking the sequences for $M C_{t}, Y_{t}$ and $p_{H, t}$ as given.

Second, consider the case where prices are set one period in advance as in Obstfeld and Rogoff (1995). Since we consider only one time-unanticipated shocks around the symmetric deterministic steady state, this simply means that prices are fixed at $t=0$ and flexible for $t \geq 1$.

Third, consider Calvo price setting, where in every period, a randomly selected fraction $1-\delta$ of firms can reset their prices. Those firms that get to reset their price choose a reset price $P_{t}^{r}$ to solve

$$
\max _{P_{t}^{r}} \sum_{k=0}^{\infty} \delta^{k}\left(\prod_{h=1}^{k} \frac{1}{1+i_{t+h}}\right)\left(P_{t}^{r} Y_{t+k \mid t}-P_{H, t} M C_{t} Y_{t+k \mid t}\right)
$$

where $Y_{t+k \mid t}=\left(\frac{P_{t}^{r}}{P_{H, t+k}}\right)^{-\epsilon} C_{t+k}$.

\subsection{Market Clearing}

Defining aggregate output to be $Y_{t}=\left(\int_{0}^{1} Y_{t}(j)^{\frac{\epsilon-1}{\epsilon}} d j\right)^{\frac{\epsilon}{\epsilon-1}}$ we obtain the goods market clearing condition

$$
Y_{t}=(1-\alpha)\left(\frac{P_{H, t}}{P_{t}}\right)^{-\eta} C_{t}+\alpha \Lambda_{H, t} \int_{0}^{1}\left(\frac{P_{H, t}}{E_{i, t} P_{F, t}^{i}}\right)^{-\gamma}\left(\frac{P_{F, t}^{i}}{P_{t}^{i}}\right)^{-\eta} C_{t}^{i} d i .
$$

Labor market clearing in the home economy is then

$$
N_{t}=\frac{Y_{t}}{A_{H, t}} \int_{0}^{1}\left(\frac{P_{t}(j)}{P_{t}}\right)^{-\epsilon} d j
$$

where $N_{t}=\int_{0}^{1} N_{t}(j) d j$. 


\subsection{Terms of Trade, Exchange Rates and UIP}

Let $E_{i, t}$ be nominal exchange rate between home and $i$ (an increase in $E_{i, t}$ is a depreciation of the home currency). Because the Law of One Price holds, we can write $P_{i, t}(j)=$ $E_{i, t} P_{i, t}^{i}(j)$ and $P_{i, t}=E_{i, t} P_{i, t}^{i}$ where $P_{i, t}^{i}(j)$ is country $i^{\prime}$ 's price of variety $j$ expressed in its own currency, and $P_{i, t}^{i}=\left(\int_{0}^{1} P_{i, t}^{i}(j)^{1-\epsilon}\right)^{\frac{1}{1-\epsilon}}$ is country $i^{\prime}$ 's domestic PPI (in terms of country $i^{\prime}$ s currency, as opposed to $P_{i, t}$ which is expressed in Home currency). We therefore have

$$
P_{F, t}=E_{t} P_{t}^{*}
$$

where

$$
P_{t}^{*}=\left(\int_{0}^{1} \Lambda_{i, t} P_{i, t}^{i 1-\gamma} d i\right)^{\frac{1}{1-\gamma}}
$$

is the world price index and

$$
E_{t}=\frac{\left(\int_{0}^{1} \Lambda_{i, t} E_{i, t}^{1-\gamma} P_{i, t}^{i 1-\gamma} d i\right)^{\frac{1}{1-\gamma}}}{\left(\int_{0}^{1} \Lambda_{i, t} P_{i, t}^{i 1-\gamma} d i\right)^{\frac{1}{1-\gamma}}}
$$

is the effective nominal exchange rate.

The effective terms of trade are defined by

$$
S_{t}=\frac{P_{F, t}}{P_{H, t}}=\left(\int_{0}^{1} \Lambda_{i, t} S_{i, t}^{1-\gamma} d i\right)^{\frac{1}{1-\gamma}}
$$

where $S_{i, t}=P_{i, t} / P_{H, t}$ is the terms of trade of home versus $i$. The terms of trade can then be expressed as

$$
S_{t}=\frac{E_{t} P_{t}^{*}}{P_{H, t}}
$$

The terms of trade can be used to rewrite the home CPI as

$$
P_{t}=P_{H, t}\left[1-\alpha+\alpha S_{t}^{1-\eta}\right]^{\frac{1}{1-\eta}}
$$

Finally we can define the real exchange rate between home and $i$ as $\mathcal{Q}_{i, t}=E_{i, t} P_{t}^{i} / P_{t}$. Similarly let the effective real exchange rate be

$$
\mathcal{Q}_{t}=\frac{E_{t} P_{t}^{*}}{P_{t}} .
$$


Absence of arbitrage requires that

$$
1+i_{t}=\frac{1+\tau_{t}}{1+\tau_{t}^{i}}\left(1+i_{t}^{i}\right) \frac{E_{i, t+1}}{E_{i, t}} .
$$

for all $i \in[0,1]$. This equation indicates that capital controls introduce a wedge in the Uncovered Interest Parity (UIP) equation-an observation that will play an important role in our analysis.

\subsection{Equilibrium Conditions with Symmetric Rest of the World}

We now summarize the equilibrium conditions. For simplicity of exposition, we focus on the case where all foreign countries are identical. Moreover, we assume that foreign countries do not impose capital controls. We denote foreign variables with a star. Taking foreign variables as given, equilibrium in the home country can be described by the following equations. We find it convenient to group these equations into two blocks, which we refer to as the demand block and the supply block.

The demand block is independent of the nature of price setting. It is composed of the Backus-Smith condition

$$
C_{t}=\Theta_{t} C_{t}^{*} \mathcal{Q}_{t}^{\frac{1}{\sigma}},
$$

where $\Theta_{t}$ is a relative Pareto weight whose evolution is given by equation (13) below, by the equation relating the real exchange rate to the terms of trade

$$
\mathcal{Q}_{t}=\left[(1-\alpha)\left(S_{t}\right)^{\eta-1}+\alpha\right]^{\frac{1}{\eta-1}}
$$

the goods market clearing condition

$$
Y_{t}=(1-\alpha)\left(\frac{\mathcal{Q}_{t}}{S_{t}}\right)^{-\eta} C_{t}+\alpha \Lambda_{H, t} S_{t}^{\gamma} C_{t}^{*}
$$

the labor market clearing condition

$$
N_{t}=\frac{Y_{t}}{A_{H, t}} \Delta_{t}
$$

where $\Delta_{t}$ is an index of price dispersion $\Delta_{t}=\int_{0}^{1}\left(\frac{P_{H, t}(j)}{P_{H, t}}\right)^{-\epsilon}$, the Euler equation

$$
1+i_{t}=\beta^{-1} \frac{C_{t+1}^{\sigma}}{C_{t}^{\sigma}} \Pi_{t+1}
$$


where $\Pi_{t}=\frac{P_{t+1}}{P_{t}}=\Pi_{H, t} \frac{S_{t}}{\mathcal{Q}_{t}} \frac{\mathcal{Q}_{t-1}}{S_{t-1}}$ is CPI inflation, the arbitrage condition between home and foreign bonds

$$
\frac{\Theta_{t+1}^{\sigma}}{\Theta_{t}^{\sigma}}=\frac{1+i_{t}}{1+i_{t}^{*}} \frac{E_{t}}{E_{t+1}}
$$

and the country budget constraint

$$
N F A_{t}=-C_{t}^{*-\sigma}\left(S_{t}^{-1} Y_{t}-\mathcal{Q}_{t}^{-1} C_{t}\right)+\beta\left(1+\tau_{t}^{*}\right) N F A_{t+1}
$$

where $N F A_{t}$ is the country's net foreign assets at $t$, which for convenience, we measure in the foreign price at home $P_{F, t}$ as the numeraire, and which we adjust by the foreign marginal utility of consumption $C_{t}^{*-\sigma}$. The country budget constraint is derived from the consumer's budget constraint after substituting out the lump-sum transfer. Under government budget balance the transfer equals the sum of the revenue from the labor tax and the tax on foreign investors, net of the revenue lost to subsidize domestic residents' investments abroad. ${ }^{3}$ We also impose a No-Ponzi condition so that we can write the budget constraint in present-value form

$$
N F A_{0}=-\sum_{t=0}^{\infty} \beta^{t}\left(\prod_{s=0}^{t-1}\left(1+\tau_{s}^{*}\right)\right) C_{t}^{*-\sigma}\left(S_{t}^{-1} Y_{t}-\mathcal{Q}_{t}^{-1} C_{t}\right) .
$$

The supply block varies with the nature of price setting. With flexible prices, it boils down to the following condition, which combines the household and firm's first-order conditions,

$$
C_{t}^{-\sigma} S_{t}^{-1} \mathcal{Q}_{t}=M \frac{1+\tau^{L}}{A_{H, t}} N_{t}^{\phi}
$$

where $M=\frac{\epsilon}{\epsilon-1}$ is the desired markup of price over marginal cost, together with the no price dispersion assumption $\Delta_{t}=1$. With one period in advance price stickiness, the only difference is that at $t=0$, all prices are fixed. This means that $S_{0}=E_{0} \frac{P_{0}^{*}}{P_{H, 0}}$ where $P_{0}^{*}$ and $P_{H, 0}$ are fixed. Finally with Calvo price setting, the supply block is more complex. It is composed of the equations summarizing the first-order condition for optimal price setting

$$
\frac{1-\delta \Pi_{H, t}^{\epsilon-1}}{1-\delta}=\left(\frac{F_{t}}{K_{t}}\right)^{\epsilon-1}
$$

${ }^{3}$ Of course, we do not require budget balance but since Ricardian equivalence holds here, all other government financing schemes have the same implications. 


$$
\begin{aligned}
K_{t} & =M \frac{1+\tau^{L}}{A_{H, t}} Y_{t} N_{t}^{\phi} \Pi_{H, t}^{\epsilon}+\delta \beta K_{t+1}, \\
F_{t} & =Y_{t} C_{t}^{-\sigma} S_{t}^{-1} \mathcal{Q}_{t} \Pi_{H, t}^{\epsilon-1}+\delta \beta F_{t+1},
\end{aligned}
$$

together with an equation determining the evolution of price dispersion

$$
\Delta_{t}=h\left(\Delta_{t-1}, \Pi_{H, t}\right),
$$

where $h(\Delta, \Pi)=\delta \Delta \Pi^{\epsilon}+(1-\delta)\left(\frac{1-\delta \Pi^{\epsilon-1}}{1-\delta}\right)^{\frac{\epsilon}{\epsilon-1}}$.

For most of the paper, we will be concerned with fixed exchange rate regimes (either pegs or currency unions) in which case we have the additional restriction that $E_{t}=E_{0}$ for all $t \geq 0$ where $E_{0}$ is predetermined.

\subsection{Steady State Labor Tax}

We allow for a constant tax on labor in each country. We pin this tax rate down by assuming that it is optimally set by each country and considering a symmetric steady state with flexible prices. ${ }^{4}$

Proposition 1 (Steady State Tax). Suppose prices are flexible, that productivity is constant across time and countries and there are no export demand shocks. Then the unique symmetric deterministic equilibrium has a constant labor tax given by $\tau^{L}=\frac{1}{M} \frac{\eta+\gamma-1}{(1-\alpha) \eta+\gamma-1}-1$ and optimal capital controls are equal to zero.

From each country's perspective, the labor tax is the result of a balancing act between offsetting the monopoly distortion of individual producers and exerting some monopoly power as a country. The two terms in the optimal tax formula reflect the two legs of this tradeoff. Reflecting the former leg of the tradeoff, the optimal labor tax is decreasing in the degree of monopoly power of individual firms $(M)$. Reflecting the second leg of the tradeoff, the optimal labor tax is increasing in the degree of openness $(\alpha)$, and decreasing in the elasticity of substitutions between home and foreign goods $(\eta)$ as well as in the elasticity of substitution between goods of different countries $(\gamma)$.

\subsection{Shocks}

In the remainder of the paper we will characterize the response to various shocks. We assume that the economy is initially at the deterministic symmetric steady state and char-

\footnotetext{
${ }^{4}$ The level of the tax is actually only relevant when we study the model under the Calvo pricing assumption. Our other results apply for any level of the tax rate.
} 
acterize the optimal use of capital controls for a country in response to various shocks. For the most part, until Section 5, we assume that other countries do not use capital controls, so that $\tau_{t}^{*}=0$ for $t \geq 0$.

We will consider the following shocks:

i. productivity shocks $\left\{A_{H, t}\right\}_{t \geq 0}$,

ii. export demand shocks $\left\{\Lambda_{H, t}\right\}_{t \geq 0}$,

iii. foreign consumption shocks $\left\{C_{t}^{*}\right\}_{t \geq 0}$,

iv. wealth shocks to $N F A_{0}$,

v. risk-premium shocks $\left\{\Psi_{t}\right\}_{t \geq 0}$.

The risk premium shock can be introduced as a separate shock or computed as a combination of the export demand shocks and consumption shocks, as we explain next.

Shocks to foreign consumption represent changes in both world interest rates and export demand for the home country. For example, a change that increases the growth rate of $\left\{C_{t}^{*}\right\}_{t \geq 0}$ implies an increase in world real interest rates. In any period $t$, the level of $C_{t}^{*}$ also affects export demand $\alpha \Lambda_{H, t} S_{t}^{\gamma} C_{t}^{*}$. Thus, a shock to interest rates that keeps export demand unchanged requires a combination of shocks to both $\left\{C_{t}^{*}\right\}_{t \geq 0}$ and $\left\{\Lambda_{H, t}\right\}_{t \geq 0}$ so that $C_{t}^{*} \Lambda_{H, t}$ is unchanged. As far as the home country is concerned, changes in interest rates that leave export demand unchanged are equivalent to shocks to the rate of return in the home country demanded by investors, due to a wedge between foreign investors and the home country. Although our model lacks uncertainty, such a wedge could stand in for the risks of investing in the home country, if these risks are not equally valued between borrowers and lenders. It may also represent investor's preferences for a particular country's bonds along the lines of portfolio-balance models a la Black (1973) and Kouri (1976). Thus, we will call this particular combination of shocks, to both $\left\{C_{t}^{*}\right\}_{t \geq 0}$ and $\left\{\Lambda_{H, t}\right\}_{t \geq 0}$, a risk premium shock $\Psi_{t}=\left(C_{t+1}^{*} / C_{t}^{*}\right)^{\sigma}$.

In the small open economy context, there are many interpretations for the wealth shock. It may capture the return on investments in risky assets, the default on debt held abroad, etc, which are not explicitly modeled. Another important interpretation for wealth shocks is fluctuations in commodity prices. Suppose in addition to the goods described previously, the Home country owns an endowment of a commodity good $X_{t}$ (e.g. oil, copper, soybeans, etc.) that it does not consume and exports to international markets taking the price $P_{X, t}$ as given in foreign currency. Thus, the budget constraint 
becomes

$$
\begin{aligned}
& P_{t} C_{t}+D_{t+1}+\int_{0}^{1} E_{i, t} D_{t+1}^{i} d i \\
& \quad \leq W_{t} N_{t}+\Pi_{t}+E_{t}^{*} P_{X, t}^{*} X_{t}+T_{t}+\left(1+i_{t-1}\right) D_{t}+\int_{0}^{1} \frac{1+\tau_{t-1}}{1+\tau_{t-1}^{i}} E_{t}^{i}\left(1+i_{t-1}^{i}\right) D_{t-1}^{i} .
\end{aligned}
$$

where $E_{t}^{*}$ is the exchange rate against the reference country for which the price $P_{X, t}^{*}$ is quoted. The only new element is the presence of $E_{t}^{*} P_{X, t}^{*} X_{t}$ on the right hand side of this constraint. The model is then summarized by the same equilibrium conditions as before if we reinterpret $N F A_{t}$ as also capturing the present value (discounting using the foreign interest rate) of the revenue from exports of this commodity. Under this interpretation, a shock to the price path $\left\{P_{X, t}^{*}\right\}_{t \geq 0}$ or the endowment path $\left\{X_{t}\right\}_{t \geq 0}$ can be captured by its impact on the present value $\sum_{t=0}^{\infty} \beta^{t} C_{t}^{*-\sigma} P_{X, t}^{*} X_{t}$ as a shock to $N F A_{0}$.

\section{Flexible, Rigid and One-Period Sticky Prices}

In this section, we study three different price setting assumptions. We first focus on two extreme cases, perfectly flexible or perfectly rigid prices. We then consider an intermediate case where prices are set one period in advance. The next section considers the canonical New Keynesian pricing assumption with staggered price setting a la Calvo.

\subsection{Flexible Prices}

We start with the case of flexible prices. The planning problem maximizes utility (1) subject to the equilibrium conditions (9), (10), (11), (12) with $\Delta_{t}=1,(15)$, and (16). The maximization takes place over $\left\{C_{t}, Y_{t}, N_{t}, S_{t}, \mathcal{Q}_{t}\right\}$ taking both domestic productivity $\left\{A_{H, t}\right\}$ and foreign variables $\left\{C_{t}^{*}, \Lambda_{H, t}\right\}$ as given.

If the sequence $\left\{A_{H, t}, C_{t}^{*}, \Lambda_{H, t}\right\}$ is constant, then the solution for $\left\{C_{t}, Y_{t}, N_{t}, S_{t}, \mathcal{Q}_{t}\right\}$ is also constant as long as the program is sufficiently convex. We have verified convexity in the Cole-Obstfeld case. We assume it also holds away from this case so that we can characterize the optimum using first order conditions, as is typically done for other Ramsey problems.

Interestingly, even with flexible prices, when the paths for $A_{H, t}$ and $\Lambda_{H, t}$ are not constant, it is generally optimal to use capital controls. Optimal capital controls can be inferred by taking the first-order conditions of the planning problem above and using the fact that $\frac{\Theta_{t+1}}{\Theta_{t}}=\left(1+\tau_{t}\right)^{\frac{1}{\sigma}}$. 
Proposition 2 (Flexible Prices). Assume prices are perfectly flexible. In general, optimal capital controls are non zero. They equal zero in the following cases:

i. for permanent shocks to productivity $A_{H, t}=A_{H}^{\prime}$ for all $t \geq 0$;

ii. for permanent export demand shocks $\Lambda_{H, t}=\Lambda_{H}^{\prime}$ for all $t \geq 0$;

iii. for permanent foreign consumption shocks $C_{t}^{*}=C^{* \prime}$ for all $t \geq 0$;

iv. for wealth shocks $N F A_{0} \neq 0$.

When $\sigma=\eta=\gamma=1$, optimal capital controls are equal to zero for any $\left\{A_{H, t}\right\}_{t \geq 0}$ and $\left\{C_{t}^{*}\right\}_{t \geq 0}$, and $\tau_{t}$ has the same sign as $\Lambda_{H, t+1}-\Lambda_{H, t}$ in response to export demand shocks, and the opposite sign as $\Psi_{t}-1$ in response to risk premium shocks.

The fact that capital controls are in general useful even though prices are flexible might seem surprising given the fact that we are considering a small open economy, with no ability to affect the world interest rate. This issue is discussed in detail in Costinot et al. (2011). The result can be understood by noting that capital controls, by allowing a country to reallocate demand intertemporally, allow this country to manipulate its terms of trade, raising them in some periods and lowering them in others. This margin is in general useful, unless of course the shocks are permanent, in which case no gain can be reached by engaging in this kind of intertemporal terms of trade manipulation. Moreover, in the Cole-Obstfeld case, it is not optimal to use capital controls in response to any path for productivity, foreign consumption shocks or wealth shocks. Capital controls are optimal in this case, however, in response to export demand shocks.

\subsection{Rigid Prices}

We now turn to the extreme opposite case, where prices are entirely rigid and fixed at their steady state values $S_{t}=\mathcal{Q}_{t}=1$ for all $t \geq 0$. The planning problem now drops as constraints equations (10) and (16); the former is dropped since it is automatically satisfied, the latter should not be imposed because firms must supply whatever is demanded at the given price. The planning problem imposes $S_{t}=\mathcal{Q}_{t}=1$ for all $t \geq 0$ in all the remaining constraints. After substituting out the constraints for consumption and labor it can be written as

$$
\max _{\left\{\Theta_{t}\right\}} \sum_{t=0}^{\infty} \beta^{t}\left[\frac{1}{1-\sigma} \Theta_{t}^{1-\sigma} C_{t}^{* 1-\sigma}-\frac{1}{1+\phi}\left(\alpha \Lambda_{H, t}+\Theta_{t}(1-\alpha)\right)^{1+\phi}\left(\frac{C_{t}^{*}}{A_{H, t}}\right)^{1+\phi}\right]
$$


subject to,

$$
\alpha \sum_{t=0}^{\infty} \beta^{t} C_{t}^{* 1-\sigma}\left[\Theta_{t}-\Lambda_{H, t}\right]=N F A_{0} .
$$

The problem is convex: it features a concave objective and linear constraint in $\Theta_{t}$. Putting a multiplier $\Gamma>0$ on the left-hand side of the budget constraint, the necessary and sufficient first-order condition is

$$
\Theta_{t}^{-\sigma}-(1-\alpha)\left(\alpha \Lambda_{H, t}+\Theta_{t}(1-\alpha)\right)^{\phi} A_{H, t}^{-(1+\phi)} C_{t}^{* \sigma+\phi}+\Gamma \alpha=0
$$

The next proposition follow immediately using this equation.

Proposition 3 (Rigid Prices). If prices are completely rigid the optimal capital controls $\tau_{t}$ are:

i. the same sign as $A_{H, t+1}-A_{H, t}$, in response to productivity shocks;

ii. the opposite sign as $\Lambda_{H, t+1}-\Lambda_{H, t}$, in response to export demand shocks;

iii. the opposite sign as $C_{t+1}^{*}-C_{t}^{*}$, in response to foreign consumption shocks;

$i v$. the opposite sign as $\Psi_{t}-1$, in response to risk premium shocks;

v. equal to zero in response to initial wealth shocks.

It is interesting to contrast the use of capital controls when prices are flexible with their use when prices are entirely rigid. There are both similarities and differences. In both cases, capital controls are not used in response to permanent productivity, export demand, or initial wealth shocks. But the use of capital controls in response to transitory shocks is quite different. For example, at least in the Cole-Obstfeld case, in response to productivity shocks or foreign consumption shocks, capital controls are zero when prices are flexible, but have the opposite sign as $C_{t+1}^{*}-C_{t}^{*}$ or the same sign as $A_{H, t+1}-A_{H, t}$ when prices are entirely rigid. In response to export demand shocks, optimal capital controls $\tau_{t}$ have the same sign as $\Lambda_{H, t+1}-\Lambda_{H, t}$ when prices are flexible, but the opposite sign when prices are entirely rigid.

A useful example is when productivity is increasing over time. In this case, optimal capital controls are zero when prices are flexible, but are positive when prices are rigid. With flexible prices, the country's price index decreases over time. This expected deflation raises the real interest rate and increases the growth rate of consumption. In the ColeObstfeld case trade is always balanced, so output increases at the same rate. With rigid prices, the real interest rate is fixed, so the growth rate of consumption is too low. Positive capital controls help by increasing the nominal interest rate. This increases the growth 
rate of consumption. In the process, the growth rate of output is also increased, but less so, implying that the trade balance moves towards surplus. Since this would not happen at the flexible price allocation without capital controls, this underscores that capital controls are a second best tool. They allow the country to regain some monetary autonomy, which provides control over the intertemporal allocation of spending. However, capital controls cannot affect the division of spending between home and foreign goods when prices are completely rigid. In other words, capital controls have no expenditure switching effects.

\subsection{One Period in Advance Price Setting}

We now assume that prices are set one period in advance, as in Obstfeld and Rogoff (1995). We continue to examine a shock that hits the economy at $t=0$. This implies that prices are effectively fixed in short run, at $t=0$ when the shock hits, but flexible thereafter $t=1,2, \ldots$

We use dynamic programming to split the planning problem into two. The problem for $t \geq 1$ given $N F A_{1}$ is identical to the case with flexible prices. Define the value function

$$
V\left(N F A_{1}\right)=\max _{\left\{C_{t}, \Theta_{t}, N_{t}, Y_{t}, S_{t}, \mathcal{Q}_{t}\right\}_{t=1}^{\infty}} \sum_{t=1}^{\infty} \beta^{t-1}\left[\frac{C_{t}^{1-\sigma}}{1-\sigma}-\frac{N_{t}^{1+\phi}}{1+\phi}\right]
$$

subject to the equilibrium conditions (9), (10), (11), (12) with $\Delta_{t}=1$, (16), for all $t \geq 1$, and the budget constraint

$$
N F A_{1}=-\sum_{s=0}^{\infty} \beta^{s} C_{s+1}^{*-\sigma}\left(S_{s+1}^{-1} Y_{s+1}-\mathcal{Q}_{s+1}^{-1} C_{s+1}\right)
$$

The $t=0$ planning problem is

$$
\max _{C_{0}, \Theta_{0}, N_{0}, Y_{0}, N F A_{1}}\left(\frac{C_{0}^{1-\sigma}}{1-\sigma}-\frac{N_{0}^{1+\phi}}{1+\phi}+\beta V\left(N F A_{1}\right)\right)
$$

subject to (9), (11), (12) with $\Delta_{0}=1$, for $t=0$, imposing $S_{0}=\mathcal{Q}_{0}=1$ in all these constraints, and the budget constraint

$$
N F A_{0}=-C_{0}^{*-\sigma}\left(Y_{0}-C_{0}\right)+\beta N F A_{1} .
$$

With one-period in ahead sticky prices, we are able to provide tight results for temporary productivity and export demand shocks, as well as for permanent productivity, 
export demand, and wealth shocks in the Cole-Obstfeld case.

Proposition 4 (Transitory Shocks with Sticky Prices). Suppose that prices are sticky one period in advance. Then optimal capital controls $\tau_{0}$ in period $t=0$

i. is a decreasing function of $A_{H, 0}$ and equals zero when $A_{H, 0}=A_{H}$ in response to a transitory productivity shock (with $A_{H, t}=A_{H}$ for all $t \geq 1$ );

ii. is an increasing function $\Lambda_{H, 0}$ and equals zero when $\Lambda_{H, 0}=1$ in response to a transitory export demand shock (with $\Lambda_{H, t}=1$ for all $t \geq 1$ ), in the limit of small time intervals $(\beta \rightarrow 1)$

iii. has the same sign as $C_{0}^{*}-C^{*}$ in response to a transitory foreign consumption shock (with $C_{t}^{*}=C^{*}$ for all $t \geq 1$ ) when $\sigma=1$;

$i v$. has the same sign as $\Psi_{t}-1$ in response to a transitory risk premium shock (with $\Psi_{t}=1$ for all $t \geq 1)$ when $\sigma=1$ in the limit of small time intervals $(\beta \rightarrow 1)$.

In all these cases, optimal capital controls $\tau_{t}$ are zero for $t \geq 1$.

Overall, this proposition echoes the findings in Proposition 3, which assumed perfectly rigid prices.

To gain some intuition for these results, consider the case of a temporary negative productivity shock in the Cole-Obstfeld case. With flexible prices, the allocation would feature constant labor, a temporary decrease in output, consumption, and exports (but with constant export revenues). At $t=0$, there would be a temporary improvement in the terms of trade brought about by an increase in the prices of home goods, and a constant wage. The nominal interest rate would be unchanged, but there would be a temporary high real interest rate brought about by expected deflation as the prices of home goods revert to their original values.

Now consider what happens with one period in advance sticky prices. If the exchange rate were flexible, we would achieve the same real allocation by temporarily raising the nominal interest rate, letting the exchange rate appreciate at $t=0$ and depreciate back at $t=1$. This can be seen as a version of the classical argument in favor of flexible exchange rate famously put forth by Friedman (1953). This adjustment recreates the temporary improvement in the terms of trade and the increase in the real interest rate. ${ }^{5}$

\footnotetext{
${ }^{5}$ The argument that one can achieve the flexible price allocation relies on the Producer Currency Pricing (PCP) assumption. With Local Currency Pricing (LCP) exchange rate movements cannot affect relative prices within a country, so they have no expenditure switching effects. As a result, the first best allocation is not available even with flexible exchange rates (Devereux and Engel, 2003). Even with LCP, however, a temporary appreciation increases the nominal, and hence real, interest rate.
} 
If the exchange rate is not flexible, then this solution cannot be attained because the terms of trade are fixed at $t=0$. Without capital controls, at $t=0$, labor temporarily increases, consumption and output are constant. Compared with the flexible price allocation, output, labor and consumption are higher. Similarly, at $t=0$, inflation and the real interest rate are unchanged and are respectively higher and lower than at the flexible price allocation. It is therefore intuitively desirable to impose positive capital controls and increase the nominal interest rate to decrease consumption at $t=0$. At $t=0$, output then also decreases, but by less than consumption. Therefore the country runs positive net exports at $t=0$, which implies higher consumption and lower output for $t \geq 1$.

This analysis underscores that capital controls are a second best instrument. They allow the country to regain some monetary autonomy and therefore some control over the intertemporal allocation of spending. However, this reallocation is costly since it introduces a wedge between the intertemporal prices for home and foreign households. Moreover, capital controls cannot affect the division of spending between home and foreign goods in the short run when prices are fixed.

Our next result deals with non-transitory shocks focusing on the Cole-Obstfeld case. The proof is contained in the appendix.

Proposition 5 (Persistent Shocks with Sticky Prices). Suppose that prices are sticky one period in advance. Consider the Cole-Obstfeld case $\sigma=\eta=\gamma=1$. Then

i. in response to any shock to the path $\left\{A_{H, t}\right\}_{t \geq 0}$ of productivity, optimal capital controls $\tau_{0}$ are an decreasing function of $A_{H, 0}$ and zero when $A_{H, 0}=A_{H}$.

ii. in response to a small enough permanent export demand shock $\Lambda_{H, t}=\Lambda_{H}$ for all $t \geq 0$ where $\Lambda_{H} \neq 1$, optimal capital controls $\tau_{0}$ are an increasing function of $\Lambda_{H}$ with $\tau_{0}=0$ when $\Lambda_{H}=1$;

iii. in response to any shock to the path $\left\{C_{t}^{*}\right\}_{t \geq 0}$ of foreign consumption, optimal capital controls $\tau_{0}$ are an increasing function of $C_{0}^{*}$ and zero when $C_{0}^{*}=C^{*}$.

iv. in response to a small enough wealth shock $N F A_{0} \neq 0$, optimal capital controls $\tau_{0}$ are an increasing function of $N F A_{0}$ and zero when $N F A_{0}=0 ;{ }^{6}$

In all these cases, optimal capital controls $\tau_{t}$ are zero for $t \geq 1$.

This proposition highlights an important insight regarding the effect of intermediate price rigidity. When prices are perfectly flexible or perfectly rigid, capital controls are not

\footnotetext{
${ }^{6}$ This last result is true more generally for any positive shock NFA $A_{0}>0$.
} 
used to respond to permanent shocks. In contrast, they are used in these circumstances when prices are sticky for just one period. Intuitively, when prices can adjust more in the long run than in the short run, permanent shocks have a different effect in the short and long run. In this sense, the result is similar to temporary shocks in the case of perfectly flexible or rigid prices, where capital controls are used.

A final remark is in order. When exchange rates are fixed our analysis and results hold under either PCP or LCP assumptions. In the PCP case flexible exchange rates would obtain the first best allocation. If unavailable, then capital controls are a useful secondbest instrument. Interestingly, in the LCP case, flexible exchange rates are not enough to obtain the first-best allocation. Indeed, when prices are completely rigid, as in the previous section, or when prices are set one period in advance, as in this section, capital controls are a perfect substitute for the exchange rate. Both operate on the UIP margin, liberating monetary policy, and both lack any expenditure switching effects. This exact equivalence will no longer hold when staggered price setting, as in the Calvo price setting case which we turn to next.

\subsection{The Role of Openness}

The degree to which a fixed exchange rate, coupled with nominal rigidities, lowers welfare depends crucially on the openness of the economy. In particular, in the closed economy limit with $\alpha \rightarrow 0$ the inefficiency stemming from the distorted prices of home versus foreign goods disappears, because the share of home goods becomes negligible. As we now show, without capital controls, this does not necessarily imply that welfare losses vanish. With the nominal interest rate pinned down by the UIP condition, monetary policy is severely constrained. As a result, intertemporal distortions remain, affecting overall consumption decisions, even in the limit. Capital controls, however, can resolve these intertemporal matters and restore welfare to the first best level in some, but not all, cases. ${ }^{7}$

Proposition 6. In the closed economy limit as $\alpha \rightarrow 0$

i. without capital controls: when prices are perfectly rigid or set one period in advance, for generic shocks to $\left\{A_{H, t}, \Lambda_{H, t}, C_{t}^{*}\right\}_{t \geq 0}$ and $\left\{\Psi_{t}\right\}_{t \geq 0}$ welfare is strictly below the level obtained with flexible prices;

ii. with capital controls:

(a) when prices are perfectly rigid:

\footnotetext{
${ }^{7}$ We omit wealth shocks because in the closed economy limit they require some rescaling. For example, one could study the limit where the initial wealth shocks are scaled so that $\frac{N F A_{0}}{\alpha}$ remains constant.
} 
i. for generic shocks to $\left\{A_{H, t}, \Lambda_{H, t}, C_{t}^{*}\right\}_{t \geq 0}$ welfare remains strictly below the level obtained with flexible prices;

ii. for risk premium shocks $\left\{\Psi_{t}\right\}_{t \geq 0}$, welfare converges to the the level obtained with flexible prices;

(b) when prices are set one period in advance: welfare converges to the level obtained with flexible prices.

The first part of the proposition, highlights an interesting property of open-economy models: as the economy becomes closed, by taking the import share to zero, we do not necessarily converge to the closed economy equilibrium. For $\alpha>0$, a fixed exchange rate ensures a unique equilibrium. In contrast, in a closed economy a fixed exchange rate amounts, through the UIP condition, to setting the interest rate at the foreign interest rate. As is well known, this closed economy model with a fixed interest rate features a continuum of equilibria. Technically, the equilibrium correspondence with a fixed exchange rate is not (lower hemi) continuous in the parameter $\alpha$ at $\alpha=0$ (it is upper hemi continuous). Less technically, it suggests caution in using a closed economy model as an approximation to an open economy. The result is of independent interest, since it is unrelated to capital controls and we are unaware of any similar result in the literature.

Why are capital controls always successful at eliminating welfare losses in the closed economy limit when prices are set for just one period but not when they are permanently fixed? By affecting the interest rate, capital controls can target any level of total consumption. Although the composition of consumption, between home and foreign goods is distorted, the associated welfare losses vanish in the limit. On first thought, this would seem to imply that optimal capital controls help approach the first best welfare as $\alpha \rightarrow 0$ quite generally. However, manipulating total consumption via capital controls requires (small) departures from trade balance (for low $\alpha$ ). This creates (small) positive or negative $N F A_{1}$. Now, when prices can adjust in the following period these (small) $N F A_{1}$ balances have a negligible effects on continuation utility. This result extends when prices are fixed for any number $N>1$ of periods. However, when prices are permanently rigid, small changes in $N F A_{1}$ amount to wealth shocks that have non-negligible effects on welfare. For low $\alpha$ and perfectly rigid prices, these small wealth shock may have large effects on the economy because inducing the necessary trade balance adjustment requires large changes in overall consumption.

This raises an interesting question: Does Calvo pricing look closer to rigid price case or the one-period-in-advance case? In the next section we show that, just as in the case of fully rigid prices, the first best is not generally attained as $\alpha \rightarrow 0$. 
An important exception is the case of risk premium shocks. From the home country's perspective, this shock amounts to a world interest rate shock. What is special is that in the closed economy limit the flexible price allocation, as well as its relative prices, are unchanged by risk premium shocks. Thus, the economy does not need to adjust to these shocks, just insulate itself from them and capital controls are able to do precisely this. Perhaps the surprising result is that this logic does not apply more generally to other shocks, such as productivity. Even in the closed economy limit, capital controls are not necessarily sufficient to obtain the flexible price allocation.

\section{Staggered Price Setting}

In this section, we study the standard New Keynesian version of the model, with staggered price setting a la Calvo. In addition to allowing for more realistic dynamics, this has two important implications for our analysis. First, it introduces a welfare cost from inflation, which is not present in the case of prices set one period in advance. Second, capital controls affect the evolution of absolute and relative prices, including the real exchange rate. Forward looking optimal policy must take this into account because prices only adjust gradually. In this sense, it may introduce a prudential concern for the temporary movement of relative prices. By this we mean that it might be optimal to use capital controls now if forcing variables (productivity, export demand, foreign consumption) are either expected to return to their steady state or remain at their steady state values (e.g. "hot money") for a while and deviate only in the future (e.g. "news shocks").

As is standard in the literature, we work with a log-linearized approximation of the model. As before, at $t=0$, the economy is hit with an unanticipated shock. It is convenient to work with a continuous time version of the model. This does not affect our results, but it is useful because it implies that no price index can jump at $t=0$ and this simplifies the derivation of initial conditions characterizing the equilibrium. We denote the instantaneous discount rate by $\rho$, and the instantaneous arrival rate for price changes by $\rho_{\delta}$.

\subsection{Summarizing the Economy and the Experiment}

We first describe the natural allocation with no intervention, defined as the allocation that prevails if prices are flexible and capital controls are not used. We then summarize the behavior of the sticky price economy with capital controls in log-deviations (gaps) from the natural allocation. For both the natural and the sticky price allocation with capital 
controls, the behavior of the rest of the world is taken as given.

We use lower cases variables to denote gaps from the symmetric deterministic steady state. We denote the natural allocation with bars, and the gaps from the natural allocation with hats.

The natural allocation. The shocks we consider are $\left\{a_{h, t}, \lambda_{h, t}, c_{t}^{*}, i_{t}^{*}, \pi_{t}^{*}\right\}_{t \geq 0}$ and NFA . Although we could imitate a risk premium shock from a combination of $c_{t}^{*}$ and $\lambda_{h, t}$, as we did in the previous section, we shall now tackle this shock separately in a later section.

We first solve for the allocation as a function of these primitive shocks using the loglinearized versions of the equilibrium conditions described in Section 2.5. One can show that the natural allocation is given by

$$
\begin{aligned}
& \bar{y}_{t}=\frac{\hat{\sigma}^{-1}(1+\phi)}{1+\phi \hat{\sigma}^{-1}} a_{h, t}+\frac{\alpha}{1+\phi \hat{\sigma}^{-1}} \lambda_{h, t}-\frac{\alpha(\omega-1)}{1+\phi \hat{\sigma}^{-1}} c_{t}^{*}-\frac{\alpha \omega}{1+\phi \hat{\sigma}^{-1}} \bar{\theta} \\
& \bar{s}_{t}=\frac{1+\phi}{1+\phi \hat{\sigma}^{-1}} a_{h, t}-\frac{\phi \alpha}{1+\phi \hat{\sigma}^{-1}} \lambda_{h, t}-\frac{\sigma+\phi}{1+\phi \hat{\sigma}^{-1}} c_{t}^{*}-\frac{\sigma+\phi(1-\alpha)}{1+\phi \hat{\sigma}^{-1}} \bar{\theta}
\end{aligned}
$$

with

$$
\bar{\theta}=\frac{\rho}{\alpha} N F A_{0}+\rho \int_{0}^{\infty} e^{-\rho t} \lambda_{h, t} d t+\frac{\left(\frac{\omega}{\sigma}-1\right) \hat{\sigma}}{1+\left(\frac{\omega}{\sigma}-1\right) \hat{\sigma}(1-\alpha)} \rho \int_{0}^{\infty} e^{-\rho t}\left(\bar{y}_{t}-y_{t}^{*}\right) d t,
$$

where $y_{t}^{*}=c_{t}^{*}$, since the world needs to run balanced trade with itself. We can also compute the natural levels of employment and consumption from the equations $\bar{y}_{t}=$ $a_{h, t}+\bar{n}_{t}$ and $\bar{c}_{t}=\bar{\theta}+c_{t}^{*}+\frac{1-\alpha}{\sigma} \bar{s}_{t}$, where we have substituted $\bar{q}_{t}=(1-\alpha) \bar{s}_{t}$ into the Backus-Smith condition. The constants are given by $v=-\log \left(1+\tau^{L}\right), \mu=\log M$, $\omega=\sigma \gamma+(1-\alpha)(\sigma \eta-1)$, and $\hat{\sigma}=\frac{\sigma}{1-\alpha+\alpha \omega}$.

The natural real interest rate $\bar{r} \bar{r}_{t}$ is defined from the home Euler equation $\dot{\bar{c}}_{t}=\sigma^{-1}\left(\bar{r} r_{t}-\right.$ $\rho)$. It is more convenient to work with the natural interest rate $\bar{r}_{t}$ defined in terms of the home good $\bar{r}_{t}=\bar{r}_{t}+\alpha \dot{\bar{s}}_{t}$. This turns out to equal

$$
\bar{r}_{t}-\rho=\frac{1+\phi}{1+\phi \hat{\sigma}^{-1}} \dot{h}_{h, t}-\frac{\alpha \phi}{1+\phi \hat{\sigma}^{-1}} \dot{\lambda}_{h, t}+\frac{\alpha(\omega-1) \phi}{1+\phi \hat{\sigma}^{-1}} \dot{c}_{t}^{*} .
$$

Using the home Euler equation $\dot{\bar{c}}_{t}=\sigma^{-1}\left(\bar{r}_{t}+\alpha \dot{\bar{s}}_{t}-\rho\right)$, the foreign Euler equation $\dot{c}_{t}^{*}=$ $\sigma^{-1}\left(i_{t}^{*}-\pi_{t}^{*}-\rho\right)$, and differentiating the Backus-Smith condition $\bar{c}_{t}=\bar{\theta}+c_{t}^{*}+\frac{1-\alpha}{\sigma} \bar{s}_{t}$, we obtain the following restriction:

$$
i_{t}^{*}-\bar{r}_{t}=-\dot{\bar{s}}_{t}+\pi_{t}^{*}
$$


Summarizing the system in gaps. The equations summarizing an equilibrium are the $\log$ linearized analogues of the equilibrium conditions derived in Section 2.5. The demand block is summarized by three equations,

$$
\begin{gathered}
\dot{\hat{y}}_{t}=\frac{1-\alpha}{\sigma}\left(i_{t}-i_{t}^{*}\right)+\hat{\sigma}^{-1}\left[i_{t}^{*}-\pi_{H, t}-\bar{r}_{t}\right], \\
\sigma \hat{\hat{\theta}}_{t}=i_{t}-i_{t}^{*}, \\
\int_{0}^{\infty} e^{-\rho t} \hat{\theta}_{t} d t=\frac{\left(\frac{\omega}{\sigma}-1\right) \hat{\sigma}}{1+\left(\frac{\omega}{\sigma}-1\right) \hat{\sigma}(1-\alpha)} \int_{0}^{\infty} e^{-\rho t} \hat{y}_{t} d t,
\end{gathered}
$$

representing the Euler equation (after substituting out for consumption using the goods market clearing condition and the Backus-Smith condition), the UIP equation and the budget constraint, respectively.

The supply block consists of one equation, the New-Keynesian Philips Curve

$$
\dot{\pi}_{H, t}=\rho \pi_{H, t+1}-\hat{\kappa} \hat{y}_{t}-\lambda \hat{\sigma} \alpha \omega \hat{\theta}_{t}
$$

where $\lambda=\rho_{\delta}\left(\rho+\rho_{\delta}\right)$ and $\hat{\kappa}=\lambda(\phi+\hat{\sigma})$.

Finally, we have the initial condition

$$
\hat{y}_{0}=(1-\alpha) \hat{\theta}_{0}-\hat{\sigma}^{-1} \bar{s}_{0}
$$

which formalizes the fact that prices are sticky so that the terms of trade are predetermined at $t=0$, i.e. that $\hat{s}_{0}=-\bar{s}_{0}$.

These equations are sufficient to pin down an equilibrium in the variables that are needed to evaluate welfare (see below). We can back out the rest of the variables as follows. The terms of trade gap $\hat{s}_{t}$ from

$$
\hat{y}_{t}=(1-\alpha) \hat{\theta}_{t}+\hat{\sigma}^{-1} \hat{s}_{t} .
$$

which combines the market clearing condition with the Backus-Smith condition. Similarly, we can back out the employment gap $\hat{n}_{t}$ and the consumption gap $\hat{c}_{t}$ from

$$
\begin{aligned}
& \hat{y}_{t}=\hat{n}_{t} \\
& \hat{y}_{t}=\hat{c}_{t}+\alpha \frac{\omega}{\sigma} \hat{s}_{t}-\alpha \hat{\theta}_{t} .
\end{aligned}
$$


Finally, capital controls can be inferred from the UIP equation

$$
\tau_{t}=i_{t}-i_{t}^{*}
$$

An experiment. Suppose we start in symmetric steady state where $i_{t}=i_{t}^{*}=\bar{r}_{t}=\rho$, $\pi_{H, t}=\pi_{t}^{*}=0, \hat{y}_{t}=0$ and $\bar{s}_{t}=0$. Now suppose there is an unexpected shock that upsets the economy's primitives $\left\{a_{h, t}, \lambda_{h, t}, c_{t}^{*}, i_{t}^{*}, \pi_{t}^{*}\right\}_{t \geq 0}$ and $N F A_{0}$. Sometimes it is useful to think directly in terms of a shock to the sequence $\left\{\bar{r}_{t}, \bar{s}_{t}, i_{t}^{*}, \pi_{t}^{*}, \lambda_{h, t}\right\}_{t \geq 0}$ and NFA. It is important to recognize that these variables are not independent since they must still satisfy the restriction $i_{t}^{*}-\bar{r}_{t}=-\dot{\bar{s}}_{t}+\pi_{t}^{*}$; this reduces the potential dimensionality of the shocks one can consider.

Note that $i_{t}, \bar{r}_{t}$ and $i_{t}^{*}$ enter the log-linearized equilibrium conditions only through two variables: $i_{t}-i_{t}^{*}$, which can be thought of as endogenous and controlled by $\dot{\hat{\theta}}_{t}=\sigma^{-1} \tau_{t}$, and $\bar{r}_{t}-i_{t}^{*}$, which is an exogenous forcing variable. The only other exogenous shock that enters this system of equations is the initial natural terms of trade $\bar{s}_{0}$. (Alternatively, given our restriction, one can think of the forcing variable as $\pi_{t}^{*}-\dot{\bar{s}}_{t}$.) Note that export demand shocks $\left\{\lambda_{h, t}\right\}_{t \geq 0}$ do not appear in this system of equations. However, as we show below, they do appear in the welfare loss function. Thus, the entire set of relevant shocks can be summarized by the path for two forcing variables $\bar{r}_{t}-i_{t}^{*}$ and $\lambda_{h, t}$ plus the initial change in the terms of trade $\bar{s}_{0}$. Of course, most shocks that affect $\bar{r}_{t}$, e.g. productivity shocks, may also affect the initial terms of trade $\bar{s}_{0}$. Given this information one can compute the equilibrium outcome for output gaps $\left\{\hat{y}_{t}\right\}_{t \geq 0}$ and inflation $\left\{\pi_{H, t}\right\}_{t \geq 0}$ without capital controls. One can also solve for the optimal allocation and capital controls. This determines $\tau_{t}=i_{t}-i_{t}^{*}$, giving the path for the domestic interest rate as $i_{t}=i_{t}^{*}+\tau_{t}$.

\subsection{Optimal Capital Controls in the Cole-Obstfeld Case}

From now on we focus on the Cole-Obstfeld case. This case is attractive for two reasons. First, with flexible prices, it is optimal not to use capital controls. Second, it is relatively easy to derive a second order approximation of the welfare function around the symmetric deterministic steady state.

Loss function. When $\sigma=\gamma=\eta=1$, we can derive a simple second order approximation of the welfare function (see Appendix A.6 for the detailed derivation). The corresponding loss function (in consumption equivalent units) up to a constant independent 
of policy can be written as

$$
(1-\alpha)(1+\phi) \int_{0}^{\infty} e^{-\rho t}\left[\frac{1}{2} \alpha_{\pi} \pi_{H, t}^{2}+\frac{1}{2} \hat{y}_{t}^{2}+\alpha_{\theta} \frac{1}{2}\left(\hat{\theta}_{t}+\bar{\theta}-\alpha_{\psi} \lambda_{h, t}\right)^{2}\right] d t,
$$

with $\alpha_{\pi}=\frac{\epsilon}{\lambda(1+\phi)}, \alpha_{\theta}=\frac{\alpha}{1+\phi}\left(\frac{2-\alpha}{1-\alpha}+1-\alpha\right), \alpha_{\psi}=\frac{1-\alpha}{\frac{2-\alpha}{1-\alpha}+1-\alpha}$ and $\bar{\theta}=\frac{\rho}{\alpha} N F A_{0}+\rho \int_{0}^{\infty} e^{-\rho t} \lambda_{h, t} d t$.

The first two terms in the loss function are familiar in New-Keynesian models and are identical to those obtained by Galí and Monacelli $(2005,2008)$. The third term in the loss function is new and captures the distortions introduced by capital controls. To understand this term, consider the In the Cole-Obstfeld case, suppose no export demand shocks and set $N F A_{0}=0$. The term then becomes $\alpha_{\theta} \frac{1}{2} \hat{\theta}_{t}^{2}$ and the country's budget constraint is simply $\alpha \int e^{-\rho t} \hat{\theta}_{t} d t=0$, which represents the present value of trade balances $-\alpha \hat{\theta}_{t}$. Capital controls reallocate total consumption intertemporally, but this reallocation is costly, i.e. $\hat{\theta}_{t}^{2}$ is minimized at $\hat{\theta}_{t}=0$.

Note that $\alpha_{\pi}$ is independent of $\alpha$ but that $\alpha_{\theta}$ goes to zero when $\alpha$ goes to zero. Hence in the closed economy limit $(\alpha \rightarrow 0)$, the cost of capital controls vanishes. The reason is that for a given path of $\hat{\theta}_{t}$, the associated trade balances $-\alpha \hat{\theta}_{t}$ vanish as $\alpha$ goes to zero, and so do the distortions associated with the wedge between home and foreign intertemporal prices.

Without shocks to export demand, optimal capital controls are equal to zero when prices are flexible. In this case, the natural allocation represents an upper bound on welfare with sticky prices. This is not necessarily true when there are shocks to export demand. In that case, optimal capital controls are not zero when prices are flexible, and the natural allocation is not necessarily and upper bound on welfare with sticky prices.

Planning problem. The planning problem is a standard optimal control problem

$$
\min _{\left\{\pi_{H, t}, \hat{y}_{t}, i_{t}, \hat{\theta}_{t}\right\}} \int_{0}^{\infty} e^{-\rho t}\left[\frac{1}{2} \alpha_{\pi} \pi_{H, t}^{2}+\frac{1}{2} \hat{y}_{t}^{2}+\alpha_{\theta} \frac{1}{2}\left(\hat{\theta}_{t}+\bar{\theta}-\alpha_{\psi} \lambda_{h, t}\right)^{2}\right] d t
$$

subject to

$$
\begin{gathered}
\dot{\pi}_{H, t}=\rho \pi_{H, t}-\hat{\kappa} \hat{y}_{t}-\lambda \alpha \hat{\theta}_{t}, \\
\dot{\hat{y}}_{t}=(1-\alpha)\left(i_{t}-i_{t}^{*}\right)-\pi_{H, t}+i_{t}^{*}-\bar{r}_{t}, \\
\dot{\hat{\theta}}_{t}=i_{t}-i_{t}^{*}, \\
\int_{0}^{\infty} e^{-\rho t} \hat{\theta}_{t} d t=0,
\end{gathered}
$$




$$
\hat{y}_{0}=(1-\alpha) \hat{\theta}_{0}-\bar{s}_{0}
$$

The corresponding first-order conditions can be found in the appendix.

To get a better feel for the planning problem, it is useful to imagine how it would change if the exchange rate were flexible. The only difference is that $i_{t}^{*}$ must now be replaced by $i_{t}^{*}+\dot{e}_{t}$, and the initial condition becomes $\hat{y}_{0}=(1-\alpha) \hat{\theta}_{0}-\bar{s}_{0}+e_{0}$ where the initial level of the exchange rate $e_{0}$ and the rate of exchange rate depreciation $\dot{e}_{t}$ are additional control variables. In the case where there are no initial wealth shocks or export demand shocks $\left(N F A_{0}=\lambda_{h, t}=0\right)$, it is clear that the solution then features $\pi_{H, t}=\hat{y}_{t}=$ $\hat{\theta}_{t}=0$ and $i_{t}=i_{t}^{*}+\frac{\bar{r}_{t}-i_{t}^{*}}{1-\alpha}$. The associated path for the exchange rate is determined by $e_{t}=\bar{s}_{t}$ for all $t$, which is equivalent to $e_{0}=\bar{s}_{0}$ and $\dot{e}_{t}=\frac{\bar{r}_{t}-i_{t}^{*}}{1-\alpha}$.

With a flexible exchange rate the natural allocation can be attained. This observation about the stabilizing role of flexible exchange rates goes back to Friedman (1953). With a fixed exchange rate, perfect macroeconomic stabilization cannot be achieved. A way to understand this is to go back to Mundell's trilemma, which states that it is impossible to have at the same time free capital flows, independent monetary policy, and a fixed exchange rate. By introducing capital controls, the home country is able to regain some monetary policy autonomy, even with a fixed exchange rate.

Flexible prices. Note that in the absence of export demand shocks, this proposition shows that welfare is necessarily lower with sticky prices and optimal capital controls than with flexible prices and no capital controls (optimal capital controls are zero with flexible prices in the absence of export demand shocks), and hence the natural allocation represents an upper bound for welfare. This is not necessarily true when there are export demand shocks. Moreover in that case, optimal capital controls with flexible prices are nonzero.

Proposition 7 (Flexible Prices). Suppose prices are completely flexible. Then optimal capital controls are

$$
\tau_{t}=\frac{\alpha_{\theta} \alpha_{\psi}}{\left(\frac{\alpha}{1+\phi}\right)^{2}+\alpha_{\theta}} \dot{\lambda}_{h, t},
$$

and the allocation can be expressed in closed form (see the appendix). ${ }^{8}$

Rigid prices. When prices are completely rigid the solution can be expressed in closed form. In Section 3, we found that the optimal $\Theta_{t}$ satisfied a simple first-order condition.

\footnotetext{
${ }^{8}$ This result also applies in the limit to flexible prices, i.e. by taking $\lambda \rightarrow \infty$ while simultaneously varying $\alpha_{\pi}=\frac{\epsilon}{\lambda(1+\phi)}$ and $\hat{\kappa}=\lambda(1+\phi)$.
} 
The closed form solution we provide next can be seen as a log-linear approximation to the solution of this first-order condition.

Proposition 8 (Rigid Prices). If prices are completely rigid optimal capital controls are

$$
\tau_{t}=\frac{1}{1-\alpha+\frac{\alpha_{\theta}}{1-\alpha}}\left(\bar{r}_{t}-i_{t}^{*}+\frac{\alpha_{\theta} \alpha_{\psi}}{1-\alpha} \dot{\lambda}_{h, t}\right)
$$

and the allocation can be expressed in closed form (see the appendix).

Consistent with the earlier Proposition 3, the formula shows that, when prices are rigid, capital controls are not used in response to a pure terms of trade shock: a permanent shock in $\bar{s}_{t}$ with no shock to $\bar{r}_{t}$ (for example, due to a permanent shock in productivity). Capital controls are used in response to natural interest rate $\left(\bar{r}_{t}\right)$ and export demand $\left(\lambda_{h, t}\right)$ shocks. This contrasts with the case of perfectly flexible prices, where capital controls are used only in response to export demand shocks. Moreover, even in the case of pure export demand shocks, the response is differently under flexible versus rigid prices.

Closed Economy limit $(\alpha \rightarrow 0)$. We can also derive the optimal allocation in closed form for the closed-economy limit $\alpha \rightarrow 0 .{ }^{9}$ It is important to understand what this limit represents. Basically, we index the solution of our planning problem (19) by $\alpha$ and look at the limit (for any $t$ ) of this solution when $\alpha \rightarrow 0$. This is not equivalent to the planning for a closed economy. The difference is that a closed economy would not face the budget constraint $\int_{0}^{\infty} \hat{\theta}_{t} e^{-\rho t} d t=0$. The closed economy solution is trivial and features perfect stabilization $\hat{y}_{t}=\pi_{H, t}=0$ with $i_{t}=\bar{r}_{t}, \dot{\hat{\theta}}_{t}=\bar{r}_{t}-i_{t}^{*}$ and $\hat{\theta}_{0}=\bar{s}_{0}$ so that $\int_{0}^{\infty} \hat{\theta}_{t} e^{-\rho t} d t=\frac{1}{\rho} \bar{s}_{0}+$ $\frac{1}{\rho} \int_{0}^{\infty}\left(\bar{r}_{t}-i_{t}^{*}\right) e^{-\rho t} d t$. As long as $\bar{s}_{0}+\int_{0}^{\infty}\left(\bar{r}_{t}-i_{t}^{*}\right) e^{-\rho t} d t \neq 0$, the constraint $\int_{0}^{\infty} \hat{\theta}_{t} e^{-\rho t} d t=0$ binds for the planning problem (19) with $\alpha \rightarrow 0$, and the solution of the planning problem does not converge to the closed economy solution. These observations are consistent with the discontinuity results in Proposition 6.

Note that $\alpha_{\pi}$ is independent of $\alpha$ and that $\alpha_{\theta}$ converges to zero.

Proposition 9 (Closed Economy Limit). In the closed economy limit as $\alpha \rightarrow 0$,

$$
\tau_{t}=\bar{r}_{t}-i_{t}^{*}+\Gamma\left(\frac{\left(1-\alpha_{\pi} \hat{\kappa}\right)^{2}}{\alpha_{\pi}(\rho-v)} e^{v t}-\frac{1}{\rho \alpha_{\pi}}\right)
$$

where

$$
v=\frac{\rho-\sqrt{\rho^{2}+4 \alpha_{\pi}^{2} \hat{\kappa}^{2}}}{2}
$$

\footnotetext{
${ }^{9}$ To take this limit, we assume that there are not initial wealth shocks so that $N F A_{0}=0$.
} 


$$
\Gamma=\left[\bar{s}_{0}+\int_{0}^{\infty} e^{-\rho t}\left(\bar{r}_{t}-i_{t}^{*}\right)\right]\left[1+\hat{\kappa}+\left(1-\alpha_{\pi} \hat{\kappa}\right)\left(\frac{1}{\rho^{2} \alpha_{\pi}}-\frac{1-\alpha_{\pi} \hat{\kappa}}{\alpha_{\pi}(\rho-v)^{2}}\right)\right] .
$$

The allocation can be expressed in closed form (see the appendix).

For any finite time $t$, the proposition provides an accurate approximation to the solution for small $\alpha$. However, as long as $\Gamma \neq 0$ capital controls do not return to zero in the long run. By contrast, for $\alpha>0$, one can show that capital controls always returns to zero in the long run. Thus, the double limits $\lim _{\alpha \rightarrow 0} \lim _{t \rightarrow \infty} \tau_{t}=0$ and $\lim _{t \rightarrow \infty} \lim _{\alpha \rightarrow 0} \tau_{t} \neq 0$ do not coincide. This reflects the fact that, as $\alpha \rightarrow 0$, capital controls converges to zero in the long run, as $t \rightarrow \infty$, but do so increasingly slowly. This is something that will be apparent in our simulations.

In the particular case where $1-\alpha_{\pi} \hat{\kappa}=0$, the solution is remarkably simple.

Corollary. In the closed economy limit $\alpha \rightarrow 0$, in the special case where $1-\alpha_{\pi} \hat{\kappa}=0$, capital controls are given by

$$
\tau_{t}=\bar{r}_{t}-i_{t}^{*}-\hat{\kappa} \frac{\Gamma}{\rho}
$$

and the allocation is constant: $\hat{y}_{t}=-\Gamma$ and $\pi_{H, t}=-\frac{1}{\rho \alpha_{\pi}} \Gamma$.

As an example, consider a permanent improvement in the terms of trade, so that $\bar{s}_{0}<$ 0 with no change in $\bar{r}_{t}$ or $i_{t}^{*}$. Then $\Gamma=\bar{s}_{0}(1+\hat{\kappa})<0$ and the solution entails positive capital controls: $\tau_{t}=-\hat{\kappa} \frac{\Gamma}{\rho}>0$. This conclusion on the use of capital controls in response to permanent productivity shocks consistent with Proposition 5 and 6 . Consider now the case of a pure (positive or negative) mean-reverting natural interest rate shock to $\bar{r}_{t}$ with $\bar{s}_{0}=0$ and $i_{t}^{*}=\rho$. In this case $\Gamma$ has the same sign as the natural interest rate shock, and so does $i_{t}-\bar{r}_{t}=\hat{\kappa} \frac{\Gamma}{\rho}$. In other words, in the closed economy limit, capital controls less than fully offset natural interest rate shocks.

This shows that the Calvo pricing case shares properties of the rigid prices case and of the one period in advance price setting case. As with one period in advance price setting, and by contrast with rigid prices, capital controls are used to accommodate permanent shocks to productivity. As with rigid prices, and by contrast with one period in advance price setting, the natural allocation is not attained in the closed economy limit.

The solution for capital controls is history dependent as long as $\Gamma \neq 0$-it depends on future and past values of the shocks. Imagine for example that productivity is unchanged for $t \in[0, T]$, is then expected to gradually decrease to a new lower level over the interval $t \in\left[T, T^{\prime}\right]$, and then remain constant for $t \in\left[T^{\prime}, \infty\right)$. Capital controls would be zero for $t \in[0, T]$ under flexible prices (Proposition 7), rigid prices (Proposition 8) or prices set one period in advance (Proposition 4). In contrast, with Calvo pricing, since $\Gamma<0$ capital 
controls are positive $\tau_{t}>0$ for $t \in[0, T]$. This illustrates a prudential nature of capital controls, which is absent without staggered price setting. ${ }^{10}$

\subsection{A Risk Premium Shock}

Many discussions of capital controls, especially in developing countries, focus on capital inflow surges that are taken to be exogenous fluctuations in investor sentiments. To capture this, we now model a risk premium shock $\psi_{t}$. The UIP equation becomes $i_{t}=i_{t}^{*}+\psi_{t}+\tau_{t}$, so that the risk premium introduces a wedge between foreign investors and the home country, in addition to capital controls. We do not attempt to model this wedge endogenously. Although our model lacks uncertainty, it could stand in for the risks of investing in the home country, if these risks are not equally valued between borrowers and lenders. It may also represent investor's preferences for a particular country's bonds along the lines of portfolio-balance models a la Black (1973) and Kouri (1976).

As we already discussed in Section 3, as far as the home country is concerned, this shock admits an equivalent representation as a combination of a shock to $\left\{C_{t}^{*}\right\}_{t \geq 0}$ with $\frac{\dot{C}_{t}^{*}}{C_{t}^{*}}=\dot{c}_{t}^{*}=\psi_{t}$ accompanied by an offsetting shock $\left\{\Lambda_{H, t}\right\}_{t \geq 0}$ to the demand for the home country's exports so that $C_{t}^{*} \Lambda_{H, t}=C^{*}$. We exploited this equivalent representation in Section 3. Here instead, we model the risk premium shock directly, which we find in many respects more enlightening. We confine ourselves to the Cole-Obstfeld case.

The natural allocation. We confine ourselves to shocks to $\psi_{t}$, setting $c_{t}^{*}=a_{t}=\pi_{t}^{*}=0$, $N F A_{0}=0$ and $i_{t}^{*}=\rho$. Without capital controls we have $\bar{\theta}_{t}=\bar{\theta}_{0}+\int_{0}^{t} \psi_{s} d s$. The natural allocation is then

$$
\begin{aligned}
& \bar{y}_{t}=-\frac{\alpha}{1+\phi} \bar{\theta}_{t}, \\
& \bar{s}_{t}=-\frac{1+\phi(1-\alpha)}{1+\phi} \bar{\theta}_{t},
\end{aligned}
$$

where the budget constraint

$$
\bar{\theta}_{0}+\int_{0}^{\infty} \psi_{t} e^{-\rho t} d t=0
$$

pins down $\bar{\theta}_{0}$. We can also compute the natural levels of employment and consumption from the equations $\bar{y}_{t}=\bar{n}_{t}$ and $\bar{c}_{t}=\bar{\theta}_{t}+(1-\alpha) \bar{s}_{t}$.

\footnotetext{
${ }^{10}$ For perfectly rigid prices, this is true as a first order approximation, i.e. it is a feature of the loglinearized solution but not of the full nonlinear solution. The solution for $\Theta_{t}$ in equation (17) depends on $\Gamma$. However, what Proposition 8 shows is that, to a first-order approximation, the ratio $\Theta_{t+1} / \Theta_{t}$ is independent of $\Gamma$.
} 
We can compute the natural interest rate

$$
\bar{r}_{t}-\rho=\frac{\alpha \phi}{1+\phi} \dot{\bar{\theta}}_{t}
$$

The natural allocation features trade imbalances. Indeed net exports are given by $\bar{n} x_{t}=-\alpha \bar{\theta}_{t}$, so that for negative risk premium shocks $\left(\psi_{t} \leq 0\right.$ for all $\left.t\right)$, the home country initially runs a trade deficit, and eventually runs a trade surplus together. Hence a negative risk premium shock leads on impact to current account deficits or "capital inflow surges". Conversely, a positive risk premium shock leads on impact to current account surpluses or "capital flights" or "sudden stops".

The planning problem in gaps. We are led to the following planning problem

$$
\min _{\left\{\pi_{H, t}, \hat{y}_{t}, i_{t}, \hat{\theta}_{t}\right\}} \frac{1}{2} \int_{0}^{\infty} e^{-\rho t}\left[\alpha_{\pi} \pi_{H, t}^{2}+\hat{y}_{t}^{2}+\alpha_{\theta}\left(\hat{\theta}_{t}+\alpha_{\psi} \bar{\theta}_{t}+\left(1-\alpha_{\psi}\right) \bar{\theta}_{0}\right)^{2}\right] d t
$$

subject to

$$
\begin{gathered}
\dot{\pi}_{H, t}=\rho \pi_{H, t}-\hat{\kappa} \hat{y}_{t}-\lambda \alpha \hat{\theta}_{t} \\
\dot{\hat{y}}_{t}=(1-\alpha)\left(i_{t}-i_{t}^{*}-\psi_{t}\right)-\pi_{H, t}+i_{t}^{*}+\psi_{t}-\bar{r}_{t}, \\
\dot{\hat{\theta}}_{t}=i_{t}-i_{t}^{*}-\psi_{t}, \\
\int_{0}^{\infty} e^{-\rho t} \hat{\theta}_{t} d t=0, \\
\hat{y}_{0}=(1-\alpha) \hat{\theta}_{0}-\bar{s}_{0} .
\end{gathered}
$$

Capital controls are given by $\tau_{t}=i_{t}-i_{t}^{*}-\psi_{t}$.

Flexible prices. With flexible prices, optimal capital controls are not zero, consistent with Proposition 2.

Proposition 10 (Risk Premium Shock, Flexible Prices). Suppose prices are completely flexible and the economy is subject to risk-premium shocks $\psi_{t}$. Then optimal capital controls are given by

$$
\tau_{t}=-\frac{\alpha_{\theta} \alpha_{\psi}}{\left(\frac{\alpha}{1+\phi}\right)^{2}+\alpha_{\theta}} \psi_{t}
$$

and the allocation can be expressed in closed form (see the appendix). ${ }^{11}$

\footnotetext{
${ }^{11}$ This result also applies in the limit to flexible prices, i.e. by taking $\lambda \rightarrow \infty$ while simultaneously varying $\alpha_{\pi}=\frac{\epsilon}{\lambda(1+\phi)}$ and $\hat{\kappa}=\lambda(1+\phi)$.
} 
Optimal capital controls are proportional to the current risk premium $\psi_{t}$ shock. The tax $\tau_{t}$ has the opposite sign from $\psi_{t}$-policy leans against the wind, reducing the nominal interest rate $i_{t}$ when $\psi_{t}>0$ and increasing it when $\psi_{t}<0$. Recall that with no intervention we have $i_{t}=i_{t}^{*}+\psi_{t}$.

Optimal capital controls also have the property of stabilizing the trade balance. Since the trade balance with intervention equals $n x_{t}=\hat{n} x_{t}+\bar{n} x_{t}=-\alpha \frac{\left(\frac{\alpha}{1+\phi}\right)^{2}+\alpha_{\theta}\left(1-\alpha_{\psi}\right)}{\left(\frac{\alpha}{1+\phi}\right)^{2}+\alpha_{\theta}} \bar{\theta}_{t}$ and without intervention equals $\bar{n} x_{t}=-\alpha \bar{\theta}_{t}$, the ratio $n x_{t} / \bar{n} x_{t}$ is constant and less than one. Optimal capital controls therefore mitigate the capital inflow surges associated with negative risk premium shocks, as well as the capital flight episodes associated with positive risk premium shocks.

Rigid prices. We can prove the analogues of Propositions 8 and 9. When prices are completely rigid, we find the following simple solution.

Proposition 11 (Risk Premium Shock, Rigid Prices). Suppose that prices are completely rigid, then optimal capital controls are given by

$$
\tau_{t}=-\frac{1-\alpha+\frac{2 \alpha}{1+\phi}}{1-\alpha+\frac{\alpha}{1+\phi} \frac{2-\alpha+(1-\alpha)^{2}}{(1-\alpha)^{2}}} \psi_{t},
$$

and the allocation can be expressed in closed form (see the appendix).

Once again, policy leans against the wind, more so than under flexible prices ( $\tau_{t}$ reacts more to $\psi_{t}$ with rigid prices than with flexible prices), and the more so, the smaller $\alpha$. Just as with flexible prices, optimal capital controls have the property of stabilizing the trade balance and the ratio $n x_{t} / \bar{n} x_{t}$ is constant, less than one, and lower than under flexible prices. As shown in the appendix, $\int_{0}^{\infty} \hat{y}_{t} e^{-\rho t} d t=0$, so that $\hat{y}_{t}$ necessarily takes both signs.

Closed economy limit $(\alpha \rightarrow 0)$. We can also look at the closed economy limit $\alpha \rightarrow 0$ for any degree of price rigidity.

Proposition 12 (Risk Premium Shock, Closed Economy Limit $\alpha \rightarrow 0$ ). In the closed economy limit $\alpha \rightarrow 0$, optimal capital controls are $\tau_{t}=-\psi_{t}$ so that the nominal interest rate is $i_{t}$ unaffected by the shock. The allocation coincides with the natural allocation: $\hat{y}_{t}=\pi_{H, t}=0$.

This result implies that optimal policy is history independent: in response to risk premium shocks it reacts directly to current shocks only. Again, optimal policy leans against the wind, but, in this case, the tax perfectly offsets the effect of the risk premium shock on 
the domestic nominal interest rate. This policy perfectly stabilizes output and inflation, attaining the first best. Hence in the closed economy limit, capital controls are the perfect tool to stabilize risk premium shocks.

Flexible exchange rate. The allocation with flexible prices can be attained with sticky prices if the exchange rate is allowed to vary. It is interesting to translate our results under this reinterpretation. Without capital controls, we denote by $\bar{e}_{t}$ the path for the natural nominal exchange rate that implements the natural allocation with zero home inflation $\pi_{H, t}=0$. Consider the case of a mean-reverting negative risk premium shock. Then the exchange rate appreciates on impact and then depreciates over time. Moreover one can show that with flexible exchange rates but in the absence of capital controls, this allocation is optimal.

However, a better outcome can be achieved by also using capital controls as in Proposition 10. The path for the nominal exchange rate $e_{t}$ that implements this allocation with zero home inflation $\pi_{H, t}=0$ is smoother with $e_{t} / \bar{e}_{t}$ constant and less than one (it actually equals $n x_{t} / \bar{n} x_{t}$ since the relevant elasticity is unitary).

This however, is still not the optimal joint use of capital controls and exchange rate policy when prices are sticky, which is the solution of a planning problem similar to the one in (20) with the following differences: first, there is an additional control variable $\dot{e}_{t}$; second, $i_{t}^{*}$ must be replaced throughout by $i_{t}^{*}+\dot{e}_{t}$; third, the initial condition must be dropped (technically it must be replaced by $\hat{y}_{0}=(1-\alpha) \hat{\theta}_{0}-\bar{s}_{0}+e_{0}$ where $e_{0}$ is an independent control variable).

Proposition 13 (Risk Premium Shock, Flexible Exchange Rate). Suppose that the exchange rate is flexible. The optimal solution features nonzero inflation $\pi_{H, t} \neq 0$ and capital controls are given by

$$
\tau_{t}=-\alpha_{\psi} \psi_{t}-\frac{\lambda \alpha}{\alpha_{\theta}} \alpha_{\pi} \pi_{H, t},
$$

and the solution can be expressed in closed form (see the appendix).

The solution is particularly simple when prices are rigid. Then capital controls are given by $\tau_{t}=-\alpha_{\psi} \psi_{t}$, and so lean more against the wind than at the allocation characterized in Proposition 10. This illustrates an interesting interaction of rigid prices and capital controls even with a flexible exchange rate. One can also show that capital controls lean less against the wind when the exchange rate is flexible than when the exchange rate is fixed.

Note also that in the closed economy limit, the allocation with optimal capital controls and optimal exchange rate, the allocation with optimal capital controls and fixed 
exchange rate, and the allocation with no capital controls but optimal exchange rate, all coincide with the flexible price allocation of the closed economy limit. Hence in the closed economy limit, capital controls and exchange rates are both perfect but equivalent tools to deal with risk premium shocks, and one of them can be dispensed with. However, away from the closed economy limit, both have a distinct role.

\subsection{Numerical Exploration}

In this section we numerically explore how capital controls are optimally used to respond to pure terms of trade shocks, pure natural interest rate shocks, mean-reverting productivity shocks and risk-premium shocks. We follow Galí and Monacelli (2005) by setting $\phi=3, \rho=0.04, \delta=1-0.75^{4}, \epsilon=6$. We report results for two degrees of openness: $\alpha=0.4$ and $\alpha=0.1$.

For our simulations, we will abstract from shocks to initial wealth and export demand $\left(N F A_{0}=\lambda_{h, t}=0\right)$ and we will assume that $\bar{r}_{t}-i_{t}^{*}=r^{\text {shock }} e^{-\rho_{r} t}$. Hence our restriction implies that this must necessarily be accompanied by a matching path for $-\dot{\bar{s}}_{t}+\pi_{t}^{*}$. This parametrization is flexible enough to accommodate, for example, pure terms of trade shocks (permanent shocks to $\bar{s}_{t}$ with no shock to $\bar{r}_{t}$ ), pure natural interest rate shock (shocks to $\bar{r}_{t}$ and $\dot{\bar{s}}_{t}$ with no shocks to $\bar{s}_{0}$ ), and any combination of the two such as mean-reverting productivity shocks. We will also look at risk premium shocks where we assume that $\psi_{t}=\psi^{\text {shock }} e^{-\rho_{\psi} t}$.

A permanent shock to the terms of trade. Our first experiment is such that $\bar{s}_{t}=-0.05$ for all $t$ and $\bar{r}_{t}=0$ for all $t$. This represents an improvement in the terms of trade that causes an appreciation in the real exchange rate. This can be traced back to a permanent shock to productivity $A_{H, t}$.

Figures 1 and 2 show the results for two values of openness, $\alpha=0.4$ and $\alpha=0.1$, respectively. The green line is the outcome without interventions (zero capital controls). The blue line is the outcome with optimal capital controls.

To gain intuition, consider first the outcome without intervention. On the one hand, if prices were fully flexible there would be an immediate and permanent upward jump in the price of home goods $P_{H}$. On the other hand, with totally rigid prices the equilibrium features a permanent rise in output and consumption. In the intermediate case, with Calvo pricing, the equilibrium features a smoother transition towards a higher price for home goods. As shown in the figure, home inflation and output gaps are positive and asymptote to zero. Due to the Cole-Obstfeld parameterization, the transition does not 
affect trade balance. Output and consumption are higher, pushing for higher imports (foreign revenue is constant), but Home goods are also cheaper, encouraging the relative consumption of home goods over imports. These two effects cancel each other out.

Turning to the optimum, Proposition 7 indicates that with flexible prices no intervention is required. With fully rigid prices, Proposition 8 implies that the optimum does nothing and accepts the permanent change in output. Interestingly, in the intermediate case, with Calvo pricing, the optimal intervention is not zero, so it is not intermediate between these two extremes of fully flexible or rigid prices. The reason for this is that, unlike the case with fully rigid prices, the effects of the shock with Calvo prices without intervention are not permanent. Capital controls can shift spending across time. Intuitively, this is not desirable if the response without intervention produces constant outcomes. But capital controls are more useful if the response without intervention is not constant. In the short run, the optimal policy intervention lowers home consumption and output relative to the no-intervention equilibrium. The drop in consumption lowers imports, which creates a trade surplus. The rest of the dynamics are simply convergence. Note the tradeoff: the optimal policy lowers output and consumption, which makes inflation lower, slowing the needed adjustment in home prices. Thus, eventually the output gap with and without intervention cross. (In fact, they cross again due to the income effect from the accumulated trade surpluses.) Note that when $\alpha$ is lower, the nominal interest rate $i_{t}$ is higher, so that capital controls are used more.

A temporary shock to the natural interest rate. Next we look at a shock to $\bar{r}_{t}$ which becomes negative (with $\bar{r}_{0}=-0.05$ ) and then mean-reverts to zero with $\bar{s}_{0}=0$. The coefficient of mean reversion is set to a relatively high value of 1 , so that the half-life of the shock is 0.7 years. This shock can be traced back to a temporary productivity shock where at $t=0$, it becomes known that productivity will be decreasing in the future.

Figures 3 and 4 show the results for two values of openness, $\alpha=0.4$ and $\alpha=0.1$, respectively. The green line is the outcome without interventions (zero capital controls). The blue line is the outcome with optimal capital controls.

Consider first the outcome without intervention. If prices were flexible, then $\pi_{H, t}=$ $i_{t}^{*}-\bar{r}_{t}$ so there would be a rise in home inflation reducing the real interest rate. Since the shock is mean reverting, inflation also reverts back to zero. With sticky prices, this inflationary process is smoothed: inflation is at first lower, then higher than the flexible price outcome. Inflation also converges to zero in the long run. Indeed, the long-run increase in the price of home goods, $P_{H}$, is the same in both cases. As a result, the home price $P_{H, t}$ with sticky prices is perpetually behind where it would be with flexible prices. In 
other words, the real exchange rate appreciates over time, but it does so faster with flexible prices. This helps explain the observed positive output gap. To further understand the behavior of the output gap, it is useful to keep in mind the long-run Euler equation $\hat{y}_{t}=\hat{y}_{\infty}-\int_{t}^{\infty}\left(i_{s}^{*}-\bar{r}_{s}-\pi_{H, s}\right) d s$ together with the fact that the long-run output gap $\hat{y}_{\infty}$ is zero. Because the long-run increase $\int_{0}^{\infty} \pi_{H, s} d s$ in $P_{H}$ is the same as under flexible prices, the output gap is initially zero. Because inflation is initially lower and eventually higher than under flexible prices, the output gap is positive throughout. However, it converges to zero, since both $i_{t}^{*}-\bar{r}_{t}$ converges to zero, by assumption and we have argued that $\pi_{H, t}$ converges to zero in the long run. This explains the hump-shaped response for output. Once again, due to the Cole-Obstfeld parameterization, the transition does not affect trade balance.

We now turn to the optimal intervention. With fully rigid prices, we have shown that capital controls set $i_{t}=i_{t}^{*}+\frac{1}{1-\alpha+\frac{\alpha_{\theta}}{1-\alpha}}\left(\bar{r}_{t}-i_{t}^{*}\right)$, displayed as a red line in the figures. This implies a subsidy on inflows, coupled with a tax on outflows, to reduce the nominal interest rate. The output gap would still be zero initially and positive throughout, but it is now smoother than without intervention. The country would initially run a trade surplus. With Calvo pricing, the solution has similar features. Additionally, there is inflation. The paths for the output gap and inflation are smoother with the optimal intervention than with no intervention. When $\alpha$ is lower, the nominal interest rate is much closer to $i_{t}^{*}+$ $\frac{1}{1-\alpha+\frac{\alpha \theta}{1-\alpha}}\left(\bar{r}_{t}-i_{t}^{*}\right)$ which is turn closer to $\bar{r}_{t}$, so that capital controls are used more. The paths for the output gap and inflation become much smoother.

A temporary shock to productivity. Finally we look at a mean-reverting negative productivity shock. The half-life of the shock is set to 3.5 years and the magnitude is such that $\bar{s}_{0}=-0.05$.

Figures 5 and 6 show the results two values of openness, $\alpha=0.4$ and $\alpha=0.1$, respectively. The green line is the outcome without interventions (zero capital controls). The blue line is the outcome with optimal capital controls. This shocks is a combination of the first two shocks (with a positive sign on the first one and a negative sign on the second one). The figures can be understood accordingly.

A temporary risk premium shock. For our experiment, we look at a shock to $\psi_{t}$ which becomes negative (with $\bar{\psi}_{0}=-0.05$ ) and then mean-reverts to zero, such that 
$\psi_{t}=\psi^{\text {shock }} e^{-\rho_{\psi} t}$. The half-life of the shock is 3.5 years. In that case, we have

$$
\bar{s}_{0}=\frac{1+\phi(1-\alpha)}{1+\phi} \frac{\psi^{\text {shock }}}{\rho+\rho_{\psi}} .
$$

Figures 7 and 8 show the results for two values of openness, $\alpha=0.4$ and $\alpha=0.1$, respectively. The green line is the outcome without interventions (zero capital controls) in gaps. The blue line is the outcome with optimal capital controls in gaps.The red line represents optimal capital controls with perfectly rigid prices. The black lines corresponds to the natural allocation in levels.

We have shown in Proposition 12 that in the closed economy limit, capital controls are a perfect stabilization tool to deal with risk premium shocks. These figures confirm that that even away from this limit, capital controls perform extremely well. Notably, the optimum with rigid price provides a good benchmark, optimal capital controls are close to the red line, especially for $\alpha=0.1$. It is also apparent that compared with the allocation with no intervention, the allocation with optimal capital controls increases net exports in the short run and decreases net exports in the long run. The natural allocation features trade deficits in the short run, and trade surpluses in the long run. Hence optimal capital controls help stabilize the trade balance. As is apparent in the figures, optimal capital controls also smooth out the real exchange rate appreciation brought about by the risk premium shock.

\subsection{Sticky Wages and Flexible Exchange Rates}

As Friedman (1953) forcefully argued, flexible exchange rates can act as a substitute for flexible prices. When prices are sticky, but wages are not, a flexible exchange rate can be used to achieve the flexible price allocation. In this way, flexible exchange rates yield perfect macroeconomic stabilization. But this is no longer possible, in general, when wages are also sticky. Perfect macroeconomic stabilization is not possible, even with a flexible exchange rate. This raises the question we address here: are capital controls optimal when prices and wages are sticky but a flexible exchange rate is managed optimally?

Assume that labor is an aggregate of different varieties $N_{t}=\left(\int_{0}^{1} N_{t}(j)^{\frac{\epsilon_{w}-1}{\epsilon_{w}}} d j\right)^{\frac{\epsilon_{w}}{\epsilon_{w}-1}}$. We denote the corresponding wage index by $W_{t}=\left(\int_{0}^{1} W_{t}(j)^{1-\epsilon_{w}} d j\right)^{\frac{1}{1-\epsilon_{w}}}$, and we let $M_{w} \equiv \frac{\epsilon_{w}}{\epsilon_{w}-1}$. Wages are set a la Calvo by households, with arrival rate $\rho_{\delta_{w}}$ for wage change opportunities.

In the Appendix we present the planning problem with sticky prices and sticky wages. 
We allow there for any path of the exchange rate $e_{t}$ expressed relative to its prior, steady state, value. It can be observed from this planning problem that, in general, there is a role for capital controls when prices and wages are sticky, even when the exchange rate is flexible. However, the role of capital controls is quite different than with a fixed exchange rate. With a flexible exchange rate, each country already has the flexibility to set its own monetary policy. However, this is not enough to perfectly stabilize the economy and hence capital controls emerge as a second best instrument.

This role for capital controls entirely disappears when prices and wages are rigid, so that optimal capital controls are zero. Similarly, in the closed economy limit, capital controls are useless in the sense that the gains from using capital controls vanish.

Proposition 14 (Sticky Prices, Wages, Flexible Exchange Rate). With sticky prices, sticky wages and a flexible exchange rate, optimal capital controls are generally nonzero. However, with completely rigid prices and wages, optimal capital controls are zero. Similarly, in the closed economy limit $\alpha \rightarrow 0$, there are no gains from using capital controls.

\subsection{Government Spending}

For comparison, we now introduce government expenditures, building more directly on Galí and Monacelli (2008). Government expenditures $G_{t}$ enter households' utility

$$
\sum_{t=0}^{\infty} \beta^{t}\left[(1-\chi) \frac{C_{t}^{1-\sigma}}{1-\sigma}+\chi \frac{G_{t}^{1-\sigma}}{1-\sigma}-\frac{N_{t}^{1+\phi}}{1+\phi}\right],
$$

where $G_{t}=\left(\int_{0}^{1} G_{t}(j)^{\frac{\epsilon-1}{\epsilon}} d j\right)$, where $j \in[0,1]$ denotes the variety of home good. Note that, following Gali and Monacelli, we assume that $G_{t}$ is spent entirely on home goods.

To keep the exposition brief, we focus on the Cole-Obstfeld case and abstract from capital controls. We move directly to a continuous time formulation of the planning problem, set up as an optimal control:

$$
\min \int_{0}^{\infty}\left[\frac{1}{2} \pi_{H, t}^{2}+\frac{1}{2} \lambda_{x}\left(\hat{c}_{t}+(1-\xi) \hat{g}_{t}\right)^{2}+\frac{1}{2} \lambda_{g} \hat{g}_{t}^{2}\right] d t
$$

subject to

$$
\begin{gathered}
\dot{\pi}_{H, t}=\rho \pi_{H, t}-\hat{\kappa}\left[\hat{c}_{t}+(1-\xi) \hat{g}_{t}\right], \\
\dot{\hat{c}}_{t}=(1-\mathcal{G})\left[-\pi_{H, t}-\left(\bar{r}_{t}-i^{*}\right)\right], \\
\hat{c}_{0}=-\bar{s}_{0}(1-\mathcal{G}) .
\end{gathered}
$$


In this program, we have used the following notation. Parameter $\mathcal{G}=\frac{G}{Y}$ is the steady state share of government spending. Let $\xi=(1+(1-\mathcal{G}) \phi)^{-1}$. The rest of the parameter are: $\hat{\kappa}=\lambda\left(\phi+\frac{1}{1-\mathcal{G}}\right)$, and the coefficients in the loss function are given by $\lambda_{x}=\frac{\hat{\kappa}}{\epsilon}$ and $\lambda_{g}=\frac{\lambda}{\epsilon} \frac{1+\phi}{\Gamma} \xi$. The variable $\hat{g}_{t}$ denotes the fiscal gap $\frac{G_{t}-\bar{G}_{t}}{Y}, \hat{y}_{t}=\log \left(Y_{t}\right)-\log \left(\bar{Y}_{t}\right)$ is the output gap and $\hat{c}_{t}=\hat{y}_{t}-\mathcal{G} \hat{g}_{t}$ is the gap in private consumption (domestic and foreign) of the home good, where bar variables denote the optimal allocation with flexible prices.

Following Werning (2012), we decompose government expenditures into two components.

$$
\begin{gathered}
\hat{g}_{t}=\hat{g}^{o p p}\left(\hat{c}_{t}\right)+\hat{g}_{t}^{\text {stim }} \\
\hat{g}^{o p p}\left(\hat{c}_{t}\right)=-\frac{\lambda_{x}(1-\xi)}{\lambda_{x}(1-\xi)^{2}+\lambda_{g}} \hat{c}_{t} \quad \text { and } \quad \hat{g}_{t}^{\text {stim }}=\frac{1-\xi}{\lambda_{x}(1-\xi)^{2}+\lambda_{g}} \hat{\kappa} \mu_{\pi, t} .
\end{gathered}
$$

The first component $\hat{g}^{o p p}\left(\hat{c}_{t}\right)$ is opportunistic spending. It is the level of expenditure that would be chosen by a government, taking the path for private consumption $\hat{c}_{t}$ as given. Its determinants are purely microeconomic. It captures the idea that it makes sense to increase government spending when the opportunity cost of labor is low. The second component, stimulus spending, $\hat{g}_{t}^{\text {stim }}$, is defined as the residual. Note that stimulus spending is zero whenever the co-state $\mu_{\pi, t}$ is zero.

We can then state the following result.

Proposition 15 (Stimulus Spending). Government spending and its components are independent of $\alpha$. Stimulus spending is initially zero: $\hat{g}_{0}^{\text {stim }}=0$. In the special case with rigid prices $\hat{\kappa}=0$ or $\hat{\kappa} \frac{1}{1-\mathcal{G}}=\lambda_{x}$ then $\hat{g}_{t}^{\text {stim }}=0$ for all $t \geq 0$.

With rigid prices, the solution is simple: government spending is purely opportunistic, and stimulus spending is equal to zero. This suggests that although government spending may play a role in the optimum, it may mostly due to the opportunistic component.

An important observation is that the planning problem for fiscal policy, expressed in gaps, is independent of the openness parameter $\alpha$, and hence so is its solution. The openness of the economy only introduces a proportional scaling factor in the associated loss function. This is an important distinction with capital controls, which became more effective as the economy became more closed.

\section{Policy Coordination}

Up to this point we have isolated the problem of a small open economy with a fixed exchange rate and characterized the optimal use of capital controls taking as given conditions in the rest of the world, including policy choices by other countries, the ensuing 
equilibrium interest rates and prices. We have therefore been silent on possible spillover effects from one country to the other. Do capital controls have beggar-thy-neighbor effects? Are there reasons to worry about "capital control wars"? Are there gains from coordinating capital controls? ${ }^{12}$

To address these questions, we now consider a coordinated solution within a monetary union using capital controls to maximize the sum of utilities. We will contrast this solution to the uncoordinated, noncooperative equilibrium, where each country acts in isolation. We focus on Calvo pricing, in the Cole-Obstfeld case, and focus on shocks to productivity shocks $\left\{a_{i, t}\right\}_{t \geq 0}$. We set export demand shocks $\left\{\lambda_{i, t}\right\}_{t \geq 0}$ and initial wealth shocks $N F A_{i, 0}$ to zero for simplicity. We should note that the Cole-Obstfeld case is known to be special regarding the role of coordination with flexible exchange rates-see for example Clarida et al. (2002). We focus on that case because with capital controls, one cannot easily find a second order approximation of the loss function away from it.

An important consideration is the level of the labor tax and whether we hold it constant in our comparison. At a symmetric steady state with no coordination on this tax, the rate satisfies $M\left(1+\tau_{L}\right)=\frac{1}{1-\alpha}$ because countries attempt to manipulate their terms of trade. In contrast, if the labor tax is coordinated then it is set at a lower rate, satisfying $M\left(1+\tau_{L}\right)=1$, because the union as a whole refrains from terms of trade manipulation.

An obvious point is to note that coordination on the labor tax alone may be welfare improving, even in a steady state without shocks. We have nothing new to say on this. Instead, we find it more interesting to study coordination on capital controls alone, taking as given the labor tax level. This comparison may also be more relevant if countries find it difficult to meddle with other countries' tax systems-which is likely since these are largely shaped by other, politically sensitive and country specific, concerns, such as inequality and redistribution. It also isolates the stabilization motives for coordination, addressing the question of how best to respond to shocks, rather than the steady-state terms-of-trade manipulation issue involved in the tax level.

\subsection{Uncoordinated Labor Tax}

We start with the case where there is no coordination on the labor tax at the symmetric steady state so that $M\left(1+\tau_{L}\right)=\frac{1}{1-\alpha}$.

We define a new reference allocation which corresponds to the flexible price allocation with no capital controls. This allocation differs from the natural allocation in Section 4,

\footnotetext{
${ }^{12}$ Korinek (2012) studies policy coordination in the context of models where capital controls are used to address pecuniary externalities in borrowing constraints.
} 
which we denoted with a single bar, which corresponded to the flexible price allocation in the home country, taking the rest of the world as given. Here instead, we impose flexible prices in every country. We denote by $\bar{s}_{t}^{i}$ the terms of trade of the corresponding allocation, where by construction $\int_{0}^{1} \overline{\bar{s}}_{t}^{i} d i=0$. Because we assume only productivity shocks, we have $\overline{\bar{s}}_{t}^{i}=a_{i, t}-\int_{0}^{1} a_{i, t} d i .^{13}$

We denote by $\hat{y}_{t}^{i}$ and $\hat{\theta}_{t}^{i}$ the deviations of $y_{t}^{i}$ and $\theta_{t}^{i}$ from their flexible price counterparts. We denote by $\tilde{y}_{t}^{i}=\hat{y}_{t}^{i}-\hat{y}_{t}^{*}$ and $\tilde{\theta}_{t}^{i}=\hat{\theta}_{t}^{i}-\hat{\theta}_{t}^{*}$ where $\hat{y}_{t}^{*}=\int_{0}^{1} \hat{y}_{t}^{i} d i$ and $\hat{\theta}_{t}^{*}=\int_{0}^{1} \hat{\theta}_{t}^{i} d i=0$ the deviations these variables from the corresponding aggregates. Note that $\hat{\theta}_{t}^{i}$ is already a normalized variable so that $\hat{\theta}_{t}^{i}=\tilde{\theta}_{t}^{i}$.

Coordination. For small $\alpha$, a simple representation of the coordinated loss function with an uncoordinated labor tax is available-it is valid only for small $\alpha$, otherwise, a second order approximation of the constraints is required:

$$
\frac{1}{2} \int_{0}^{\infty} \int_{0}^{1} e^{-\rho t}\left[\alpha_{\pi}\left(\tilde{\pi}_{H, t}^{i}+\pi_{t}^{*}\right)^{2}+\left(\tilde{y}_{t}^{i}+\hat{y}_{t}^{*}\right)^{2}+\alpha_{\theta}\left(\tilde{\theta}_{t}^{i}\right)^{2}-\frac{2 \alpha}{(1-\alpha)(1+\phi)}\left(\hat{y}_{t}^{*}+\tilde{y}_{t}^{i}\right)\right] d i d t .
$$

Intuitively, the extra linear term $\frac{2 \alpha}{(1-\alpha)(1+\phi)}\left(\hat{y}_{t}^{*}+\tilde{y}_{t}^{i}\right)$ can be traced back to the fact that the that the coordinated solution abstains from terms of trade manipulation. As a result the objective acquires a preference for higher output, leading to a classical inflationary bias.

Using the fact that $\int_{0}^{1} \tilde{y}_{t}^{i} d i=\int_{0}^{1} \tilde{\pi}_{H, t}^{i} d i=0$, we are led to the following coordinated planning problem:

$$
\begin{aligned}
\min \frac{1}{2} \int_{0}^{\infty} \int_{0}^{1} e^{-\rho t}\left[\alpha_{\pi}\left(\tilde{\pi}_{H, t}^{i}\right)^{2}+\left(\tilde{y}_{t}^{i}\right)^{2}\right. & +\alpha_{\theta}\left(\tilde{\theta}_{t}^{i}\right)^{2} \\
& \left.+\alpha_{\pi}\left(\pi_{t}^{*}\right)^{2}+\left(\hat{\hat{y}}_{t}^{*}\right)^{2}-\frac{2 \alpha}{(1-\alpha)(1+\phi)} \hat{\hat{y}}_{t}^{*}\right] d i d t
\end{aligned}
$$

subject to

$$
\begin{gathered}
\dot{\tilde{\pi}}_{H, t}^{i}=\rho \tilde{\pi}_{H, t}^{i}-\hat{\kappa} \tilde{y}_{t}^{i}-\lambda \alpha \tilde{\theta}_{t,}^{i} \\
\dot{\tilde{y}}_{t}^{i}=(1-\alpha) \dot{\tilde{\theta}}_{t}^{i}-\tilde{\pi}_{H, t}^{i}-\dot{\bar{s}}_{t}^{i}, \\
\int_{0}^{\infty} e^{-\rho t} \tilde{\theta}_{t}^{i} d t=0, \\
\tilde{y}_{0}^{i}=(1-\alpha) \tilde{\theta}_{0}^{i}-\overline{\bar{s}}_{0}^{i},
\end{gathered}
$$

\footnotetext{
${ }^{13}$ Although we do not need it for our analysis, not that the natural interest rate is given by and $\overline{\bar{r}}_{t}^{i}=\dot{a}_{i, t}$.
} 


$$
\begin{gathered}
\int_{0}^{1} \tilde{y}_{t}^{i} d i=0, \\
\int_{0}^{1} \tilde{\pi}_{H, t}^{i} d i=0, \\
\dot{\pi}_{t}^{*}=\rho \pi_{t}^{*}-\hat{\kappa} \hat{y}_{t}^{*},
\end{gathered}
$$

where the minimization is over the variables $\tilde{\pi}_{H, t}^{i}, \pi_{t}^{*}, \tilde{y}_{t}^{i}, \hat{y}_{t}^{*}, \tilde{\theta}_{t}^{i}$. Note that since $\int_{0}^{1} \overline{\bar{s}}_{t}^{i} d i=0$, the constraints imply that $\int_{0}^{1} \tilde{\theta}_{t}^{i} d i=0$. This pins down controls through the equation $\dot{\tilde{\theta}}_{t}^{i}=\tau_{t}^{i}$.

It follows that we can break down the planning problem into two parts. First, there is an aggregate planning problem determining the average output gap and inflation $\hat{y}_{t}^{*}$ and $\pi_{t}^{*}$

$$
\min \frac{1}{2} \int_{0}^{\infty} e^{-\rho t}\left[\alpha_{\pi}\left(\pi_{t}^{*}\right)^{2}+\left(\hat{\hat{y}}_{t}^{*}\right)^{2}-\frac{2 \alpha}{(1-\alpha)(1+\phi)} \hat{y}_{t}^{*}\right] d t
$$

subject to (28).

Second, there is a disaggregated planning problem determining deviations from the aggregates for output gap, home inflation and consumption smoothing, $\tilde{y}_{t}^{i}, \tilde{\pi}_{H, t}^{i}$ and $\tilde{\theta}_{t}^{i}$

$$
\min \frac{1}{2} \int_{0}^{\infty} \int_{0}^{1} e^{-\rho t}\left[\alpha_{\pi}\left(\tilde{\pi}_{H, t}^{i}\right)^{2}+\left(\tilde{y}_{t}^{i}\right)^{2}+\alpha_{\theta}\left(\tilde{\theta}_{t}^{i}\right)^{2}\right] d i d t
$$

subject to (22), (23), (24), (25), (26), and (27). Consider dropping the last two constraints. The resulting relaxed planning problem can be broken down into separate component planning problems for each country $i \in[0,1]$

$$
\min \frac{1}{2} \int_{0}^{\infty} e^{-\rho t}\left[\alpha_{\pi}\left(\tilde{\pi}_{H, t}^{i}\right)^{2}+\left(\tilde{y}_{t}^{i}\right)^{2}+\alpha_{\theta}\left(\tilde{\theta}_{t}^{i}\right)^{2}\right] d t
$$

subject to (22), (23), (24), (25).

No coordination. With no coordination, each country $i \in[0,1]$ takes the evolution of aggregates as given and solves the following uncoordinated component planning problem:

$$
\min \frac{1}{2} \int_{0}^{\infty} e^{-\rho t}\left[\alpha_{\pi}\left(\tilde{\pi}_{H, t}^{i}\right)^{2}+2 \alpha_{\pi} \pi_{t}^{*} \tilde{\pi}_{H, t}^{i}+\left(\tilde{y}_{t}^{i}\right)^{2}+2 \hat{y}_{t}^{*} \tilde{y}_{t}^{i}+\alpha_{\theta}\left(\tilde{\theta}_{t}^{i}\right)^{2}\right] d t
$$

subject to (22), (23), (24), (25), where the minimization is over the variables $\tilde{\pi}_{H, t}^{i}, \tilde{y}_{t}^{i}, \tilde{\theta}_{t}^{i}$, taking $\hat{y}_{t}^{*}$, and $\pi_{t}^{*}$ as given. As usual, capital controls in country $i$ can be computed by $\tau_{t}^{i}=\dot{\tilde{\theta}}_{t}^{i}$. Note that the path for aggregates $\left\{\hat{\hat{y}}_{t}^{*}, \pi_{t}^{*}\right\}_{t \geq 0}$ affects the solution to this problem 
solely through linear terms in the objective function.

A central monetary authority, by setting monetary policy, can choose aggregates $\left\{\hat{y}_{t}^{*}, \pi_{t}^{*}\right\}$ subject to the following constraints. First, it must ensure that the solutions to the uncoordinated component planning problems satisfy $\int_{0}^{1} \tilde{y}_{t}^{i} d i=0$ and $\int_{0}^{1} \tilde{\pi}_{H, t}^{i} d i=0$. This amounts to verifying a fixed point, that aggregates are actually equal to their proposed path. Second, it must ensure that the aggregate Phillips curve is verified, $\dot{\pi}_{t}^{*}=\rho \pi_{t}^{*}-\hat{\kappa} \hat{y}_{t}^{*}$. Both requirements define a set $\mathcal{F}$ of feasible aggregate outcomes $\left\{\hat{y}_{t}^{*}, \pi_{t}^{*}\right\}_{t \geq 0}$. The set is a linear space, which we characterize in closed form below. Which aggregate outcome in the feasibility occurs depends on the objective of the central monetary authority. For example, we can examine the Ramsey problem where the central monetary authority seeks to maximize aggregate welfare, taking into account that capital controls are set uncooperatively. For small $\alpha$, this can be represented as the same planning problem as (29) but where the constraint set is the feasible set $\mathcal{F}$ instead of (28).

Proposition 16 (Coordination vs. No Coordination with Uncoordinated Labor Tax). Suppose that countries do not coordinate on the labor tax. At the coordinated solution, the aggregates solve the aggregate planning problem (29): $\hat{y}_{t}^{*}=\frac{\alpha}{(1-\alpha)(1+\phi)} e^{\nu t}$ and $\pi_{t}^{*}=\frac{\alpha}{(1-\alpha)(1+\phi)} \frac{\hat{\kappa}}{\rho-v} e^{v t}$ for all $t \geq 0$, where $v=\frac{\rho-\sqrt{\rho^{2}+4 \hat{\kappa} \alpha_{\pi}}}{2}$. At the uncoordinated solution, the aggregates $\left\{\hat{y}_{t}^{*}, \pi_{t}^{*}\right\}_{t \geq 0}$ are in the feasible set $\mathcal{F}$ if and only if

$$
\begin{aligned}
& \dot{\hat{y}}_{t}^{*}=-\frac{\hat{\kappa}}{\rho} \hat{\hat{y}}_{0}^{*}-\frac{\lambda \alpha+(\hat{\kappa}(1-\alpha)+\lambda \alpha) \hat{\kappa} \alpha \pi}{\hat{\kappa}(1-\alpha)} \pi_{t}^{*}, \\
& \dot{\pi}_{t}^{*}=\rho \pi_{t}^{*}-\hat{\kappa} \hat{\hat{y}}_{t}^{*} .
\end{aligned}
$$

In particular, $\hat{y}_{t}^{*}=\pi_{t}^{*}=0$ for all $t \geq 0$ is feasible but $\hat{y}_{t}^{*}=\frac{\alpha}{(1-\alpha)(1+\phi)} e^{v t}$ and $\pi_{t}^{*}=$ $\frac{\alpha}{(1-\alpha)(1+\phi)} \frac{\hat{\kappa}}{\rho-v} e^{v t}$ for all $t \geq 0$ is not. Both at the coordinated and at the uncoordinated solutions, the disaggregated variables $\tilde{\pi}_{H, t}^{i}, \tilde{y}_{t}^{i}, \tilde{\theta}_{t}^{i}$ solve the component planning problems (31).

The propositions shows that the feasible set $\mathcal{F}$ is of dimension one, parameterized by $\hat{y}_{0}^{*}$. For each $\hat{y}_{0}^{*}$ there is a unique $\pi_{0}^{*}$ such that the solution of the $2 \times 2$ differential system is bounded. Indeed, the solution converges to a steady state that varies with $\hat{y}_{0}^{*}$ and satisfies $\rho \pi_{\infty}^{*}=\hat{\kappa} \hat{y}_{\infty}^{*}$. In particular, unless $\hat{y}_{0}^{*}=0$ then we have $\hat{y}_{\infty}^{*} \neq 0$ and $\pi_{\infty}^{*} \neq 0$. More precisely, one can show that if $\hat{y}_{0}^{*}$ is positive then $\hat{y}_{\infty}^{*}$ and $\pi_{\infty}^{*}$ are negative, and vice versa if $\hat{y}_{0}^{*}$ is negative.

Therefore, it is impossible to stimulate output throughout at a feasible solution. This observation is useful to apprehend the Ramsey problem where the central monetary authority seeks to maximize aggregate welfare, taking into account that capital controls 
are set uncooperatively, since the objective function incorporate a linear term in output. Indeed, one can show that in the particular case where the following condition holds $\frac{\rho}{\rho-v}=\frac{1-\alpha}{\alpha \frac{1+\alpha \pi \hat{\kappa}}{1+\phi}+(1-\alpha)\left(\alpha_{\pi} \hat{\kappa}-1\right)}$, the solution of the Ramsey problem is $\hat{y}_{t}^{*}=\pi_{t}^{*}=0$ for all $t \geq 0 .{ }^{14}$

Note also that the coordinated solution converges to $\hat{y}_{\infty}^{*}=\pi_{\infty}^{*}=0$ in the long run. Since $\hat{y}_{t}^{*}=\pi_{t}^{*}=0$ is feasible, this shows that in a sense, the benefits of coordination are temporary since they are concentrated in the short run.

\subsection{Coordinated Labor Tax}

We now consider the case where there is coordination on the labor tax at the symmetric steady state so that $M\left(1+\tau_{L}\right)=1$. With a slight abuse of notation, we keep denoting with a double bar the corresponding flexible price allocation with no capital controls. We denote with a double hat the gap of a variable from its flexible price counterpart. We denote with a tilde a variable minus its mean across countries. For example $\tilde{y}_{t}^{i}=\hat{\hat{y}}_{t}^{i}-\hat{\hat{y}}_{t}^{*}$ represents the deviation of country $i$ 's output gap from the corresponding aggregate.

We only summarize the results since the analysis is quite similar to the uncoordinated labor tax case (the details can be found in the appendix). Starting with the coordinated solution, we can still decompose the coordinated planning problem into an aggregate planning problem that determines the aggregates the aggregates $\left\{\hat{y}_{t}^{*}, \pi_{t}^{*}\right\}_{t \geq 0}$ and a disaggregated planning problem that determines the disaggregated variables $\tilde{\pi}_{H, t}^{i}, \tilde{y}_{t}^{i}, \tilde{\theta}_{t}^{i}$. The disaggregated planning problem can then be relaxed and decomposed into independent component planning problems. The uncoordinated solution can be described by two requirements: that the aggregates $\left\{\hat{y}_{t}^{*}, \pi_{t}^{*}\right\}_{t \geq 0}$ be in a feasibility set $\mathcal{F}^{\prime}$ for and that the disaggregated variables $\tilde{\pi}_{H, t}^{i}, \tilde{y}_{t}^{i}, \tilde{\theta}_{t}^{i}$ solve the component planning problem.

There are differences with the uncoordinated labor tax case in the objective functions for these programs. First, the coefficient on $\frac{1}{2}\left(\tilde{\theta}_{t}^{i}\right)^{2}$ in the coordinated planning problem, the disaggregated planning problem and the component planning problem, is $(1-\alpha) \alpha_{\theta}$ instead of $\alpha_{\theta}$. Second now there is no linear term in $-\frac{2 \alpha}{(1-\alpha)(1+\phi)} \hat{y}_{t}^{*}$ in the aggregate planning problem, but there is a linear term $\frac{2 \alpha}{1+\phi} \tilde{y}_{t}^{i}$ in the problem solved by an individual country under the uncoordinated solution.

Proposition 17 (Coordination vs. No Coordination with Coordinated Labor Tax). Suppose that countries coordinate on the labor tax. At the coordinated solution, the aggregates solve the

\footnotetext{
${ }^{14}$ When the condition is not verified, the solution of the Ramsey problem is different: it might be optimal to have $\hat{y}_{0}^{*}$ positive or negative. See the appendix for the full solution.
} 
aggregate planning problem: $\hat{y}_{t}^{*}=0$ and $\pi_{t}^{*}=0$ for all $t \geq 0$. At the uncoordinated solution, the aggregates $\left\{\hat{y}_{t}^{*}, \pi_{t}^{*}\right\}_{t \geq 0}$ are in the feasible set $\mathcal{F}^{\prime}$ if and only if

$$
\begin{aligned}
& \dot{\hat{y}}_{t}^{*}=-\frac{1}{\rho} \frac{\alpha}{1+\phi} \frac{\lambda \alpha}{1-\alpha}-\frac{1}{\rho} \hat{\kappa} \hat{y}_{0}^{*}-\frac{1}{(1-\alpha) \hat{\kappa}}\left(\lambda \alpha+(\hat{\kappa}(1-\alpha)+\lambda \alpha) \alpha_{\pi} \hat{\kappa}\right) \pi_{t}^{*}, \\
& \dot{\pi}_{t}^{*}=\rho \pi_{t}^{*}-\hat{\kappa} \hat{y}_{t}^{*} .
\end{aligned}
$$

In particular, $\hat{y}_{t}^{*}=-\frac{\left(\frac{\alpha}{1+\phi}\right)^{2} \frac{1}{1-\alpha}}{1+\frac{\lambda \alpha+(\hat{\kappa}(1-\alpha)+\lambda) \alpha \pi \hat{\kappa}}{(1-\alpha) \hat{\kappa}}}$ and $\pi_{t}^{*}=\frac{\hat{\kappa}}{\rho} \hat{y}_{t}^{*}$ for all $t \geq 0$ is feasible but $\hat{y}_{t}^{*}=\pi_{t}^{*}=0$ for all $t \geq 0$ is not. Both at the coordinated and at the uncoordinated solutions, the disaggregated variables $\tilde{\pi}_{H, t}^{i}, \tilde{y}_{t}^{i}, \tilde{\theta}_{t}^{i}$ solve the component planning problems .

\subsection{The Role of Coordination}

Propositions 16 and 17 help understand the role of coordination. For a given labor tax, the aggregates $\hat{y}_{t}^{*}$ and $\pi_{t}^{*}$ associated with the coordinated solution and the uncoordinated solutions differ. Indeed, for a given labor tax, the aggregates corresponding to the coordinated solution are not feasible. By contrast, for a given labor tax, the disaggregated variables $\tilde{\pi}_{H, t}^{i}, \tilde{y}_{t}^{i}, \tilde{\theta}_{t}^{i}$ (and hence capital controls) coincide at the coordinated and uncoordinated solutions. They depend only on the labor tax, and not on whether countries coordinate or not for a given labor tax. In other words, the lack of coordination impacts the solution by restricting the set of feasible aggregate outcomes, but does not impact the disaggregated variables and the associated capital controls for any feasible aggregate outcome.

Observe that the feasible sets $\mathcal{F}$ and $\mathcal{F}^{\prime}$ are completely independent of the shocks $\bar{s}_{t}^{i}$. A interesting consequence of this observation is that the gains from coordination are completely independent of the amount of heterogeneity across countries $\bar{s}_{t}^{i}$.

\section{References}

Bianchi, Javier, "Overborrowing and Systemic Externalities in the Business Cycle," American Economic Review, December 2011, 101 (7), 3400-3426.

- and Enrique G. Mendoza, "Overborrowing, Financial Crises and 'Macro-prudential' Taxes," NBER Working Papers 16091, National Bureau of Economic Research, Inc June 2010. 
Black, Stanley, "International Money Markets and Flexible Exchange Rates," Studies in International Finance, 1973, (25).

Caballero, Ricardo J. and Arvind Krishnamurthy, "International and domestic collateral constraints in a model of emerging market crises," Journal of Monetary Economics, December 2001, 48 (3), 513-548.

_ and _ , "Smoothing sudden stops," Journal of Economic Theory, November 2004, 119 (1), $104-127$.

Calvo, Guillermo A., "Capital Flows and Capital-Market Crises: The Simple Economics of Sudden Stops," Journal of Applied Economics, November 1998, 0, 35-54.

Clarida, Richard, Jordi Gali, and Mark Gertler, "A Simple Framework for International Monetary Policy Analysis," Journal of Monetary Economics, July 2002, 49 (5), 879-904.

Cole, Harold L. and Maurice Obstfeld, "Commodity Trade and International Risk Sharing : How Much Do Financial Markets Matter?," Journal of Monetary Economics, 1991, 28 (1), 3-24.

Corsetti, Giancarlo, Luca Dedola, and Sylvain Leduc, "Optimal Monetary Policy in Open Economies," in Benjamin M. Friedman and Michael Woodford, eds., Handbook of Monetary Economics, Vol. 3 of Handbook of Monetary Economics, Elsevier, October 2010, chapter 16, pp. 861-933.

Costinot, Arnaud, Guido Lorenzoni, and Ivan Werning, "A Theory of Capital Controls as Dynamic Terms-of-Trade Manipulation," NBER Working Papers 17680, National Bureau of Economic Research, Inc December 2011.

Devereux, Michael B. and Charles Engel, "Monetary Policy in the Open Economy Revisited: Price Setting and Exchange-Rate Flexibility," Review of Economic Studies, October 2003, 70 (4), 765-783.

Fleming, Marcus, "Domestic Financial Policies under Fixed and under Floating Exchange Rates," IMF staff papers, 1962, 9 (3), 369-380.

Friedman, Milton, "The Case for Flexible Exchange Rates," in "Essays in Positive Economics," University of Chicago Press, 1953, pp. 157-203.

Galí, Jordi and Tommaso Monacelli, "Monetary Policy and Exchange Rate Volatility in a Small Open Economy," Review of Economic Studies, 07 2005, 72 (3), 707-734. 
_ and _ , "Optimal monetary and fiscal policy in a currency union," Journal of International Economics, September 2008, 76 (1), 116-132.

Jeanne, Olivier, "Capital Account Policies and the Real Exchange Rate," February 2011. Mimeo, Johns Hopkins University.

- and Anton Korinek, "Excessive Volatility in Capital Flows: A Pigouvian Taxation Approach," American Economic Review, May 2010, 100 (2), 403-407.

Keynes, J.M., “The German transfer problem," The Economic Journal, 1929, 39 (153), 1-7.

Korinek, Anton, "The New Economics of Prudential Capital Controls: A Research Agenda," IMF Economic Review, August 2011, 59 (3), 523-561.

_ , "Capital Controls and Currency Wars," 2012. Mimeo, University of Maryland.

Kouri, Pentti, "The Exchange Rate and the Balance of Payment in the Short Run and in the Long Run: A Monetary Approach," The Scandinavian Journal of Economics, 1976, 78 (2), 280-304.

Martin, Alberto and Filippo Taddei, "International Capital Flows and Credit Market Imperfections: a Tale of Two Frictions," 2010. Collegio Carlo Alberto.

Mendoza, Enrique G., "Sudden Stops, Financial Crises, and Leverage," American Economic Review, December 2010, 100 (5), 1941-66.

Mundell, Robert, "Capital Mobility and Stabilization Policy under Fixed and Flexible Exchange Rates," Canadian Journal of Economic and Political Science, 1963, 29 (4), 475-485.

Neumeyer, Pablo A. and Fabrizio Perri, "Business cycles in emerging economies: the role of interest rates," Journal of Monetary Economics, March 2005, 52 (2), 345-380.

Obstfeld, Maurice and Kenneth Rogoff, "Exchange Rate Dynamics Redux," Journal of Political Economy, June 1995, 103 (3), 624-60.

Ohlin, B., "The reparation problem: a discussion," Economic Journal, 1929, 39 (154), 172182.

Ostry, Jonathan D., Atish R. Ghosh, Karl Habermeier, Marcos Chamon, Mahvash S. Qureshi, and Dennis B.S. Reinhardt, "Capital Inflows: The Role of Controls," 2010. IMF Staff Position Note. 
Schmitt-Grohe, Stephanie and Martin Uribe, "Prudential Policies for Peggers," NBER Working Papers 18031, National Bureau of Economic Research, Inc June 2012.

Werning, Ivan, "Managing a Liquidity Trap: Monetary and Fiscal Policy," 2012. NBER Working Papers.

\section{A Appendix}

\section{A.1 Proof of Proposition 1}

To solve for this optimal labor subsidy it is useful to consider the following relaxed problem, which allows taxes to vary over time. As it turns out, at a steady state, the optimal tax is constant. Assume that the world is at a symmetric deterministic steady state. Each country takes the rest of the world as given and uses a time-varying tax on capital inflow and subsidy on capital outflow controls $\tau_{t}$ and labor tax $\tau_{L, t}$ to maximize the welfare of its households.

For example, the home country solves

$$
\max _{\left\{C_{t}, Y_{t}, N_{t}, \Theta_{t}, \mathcal{Q}_{t}, S_{t}\right\}} \sum_{t=0}^{\infty}\left[\frac{C_{t}^{1-\sigma}}{1-\sigma}-\frac{N_{t}^{1+\phi}}{1+\phi}\right]
$$

subject to

$$
\begin{gathered}
C_{t}=\Theta_{t} C^{*} \mathcal{Q}_{t}^{\frac{1}{\sigma}}, \\
Y_{t}=C^{*}\left[(1-\alpha) \mathcal{Q}_{t}^{\frac{1}{\sigma}-\eta} S_{t}^{\eta} \Theta_{t}+\alpha S_{t}^{\gamma}\right], \\
\mathcal{Q}_{t}=\left[(1-\alpha) S_{t}^{\eta-1}+\alpha\right]^{\frac{1}{\eta-1}}, \\
N_{t}=\frac{Y_{t}}{A}, \\
0=\sum_{t=0}^{\infty} \beta^{t} C^{*-\sigma}\left(S_{t}^{-1} Y_{t}-\mathcal{Q}_{t}^{-1} C_{t}\right) .
\end{gathered}
$$


We can then back out the optimal labor tax using and the optimal capital controls using

$$
\begin{aligned}
S_{t}^{-1} \Theta_{t}^{-\sigma} C^{*-\sigma} & =M \frac{1+\tau_{t}^{L}}{A} N_{t}^{\phi}, \\
\frac{\Theta_{t+1}}{\Theta_{t}} & =\left(1+\tau_{t}\right)^{\frac{1}{\sigma}} .
\end{aligned}
$$

Using this program we can prove the following.

Assuming the optimization problem is sufficiently convex, stationarity implies that it is optimal to pick constant values for $\left\{C_{t}, Y_{t}, N_{t}, \Theta_{t}, \mathcal{Q}_{t}, S_{t}\right\}$. This immediately implies that $\tau_{t}=0$ and that $\tau_{L}$ is constant.

Dropping the $t$ subscripts and substituting some of the constraints, we can rewrite the planning problem as

$$
\max _{S} \frac{\left[(1-\alpha)(S)^{\eta-1}+\alpha\right]^{\frac{(1-\sigma) \eta}{\eta-1}} S^{(1-\sigma)(\gamma-1)} C^{* 1-\sigma}}{1-\sigma}-\frac{1}{1+\phi} \frac{C^{* 1+\phi}}{A^{1+\phi}}\left[(1-\alpha)(S)^{\eta-1}+\alpha\right]^{1+\phi} S^{(1+\phi) \gamma}
$$

This yields a function $S\left(C^{*}\right)$. We then need to solve for $S\left(C^{*}\right)=1$. We can then back out the corresponding $\tau^{L}$ from the labor-leisure condition.

We find

$$
\begin{array}{r}
0=\left[\frac{(1-\alpha) \eta S^{\eta-1}}{S\left[(1-\alpha)(S)^{\eta-1}+\alpha\right]}+\frac{(\gamma-1)}{S}\right]\left[(1-\alpha)(S)^{\eta-1}+\alpha\right]^{\frac{(1-\sigma) \eta}{\eta-1}} S^{(1-\sigma)(\gamma-1)} C^{* 1-\sigma} \\
-\left[\frac{(\eta-1) S^{\eta-1}}{S\left[(1-\alpha)(S)^{\eta-1}+\alpha\right]}+\frac{\gamma}{S}\right] \frac{C^{* 1+\phi}}{A^{1+\phi}}\left[(1-\alpha)(S)^{\eta-1}+\alpha\right]^{1+\phi} S^{(1+\phi) \gamma}
\end{array}
$$

We now impose $S=1$ and solve for $C^{*}$

$$
0=[(1-\alpha) \eta+(\gamma-1)] C^{* 1-\sigma}-[(\eta-1)+\gamma] \frac{C^{* 1+\phi}}{A^{1+\phi}}
$$

i.e.

$$
\frac{C^{* \phi+\sigma}}{A^{1+\phi}}=\frac{(1-\alpha) \eta+\gamma-1}{\eta+\gamma-1}
$$

We can now plug this back in the labor-leisure condition

$$
1=M\left(1+\tau^{L}\right) \frac{C^{* \phi+\sigma}}{A^{1+\phi}}
$$


to obtain the proposition.

\section{A.2 Proof of Proposition 2}

The first part of the proposition follows immediately from the planning problem, provided the program is sufficiently convex, as we assume. We therefore focus on the ColeObstfeld case. The planning problem simplifies to

$$
\max _{\left\{\Theta_{t}, S_{t}\right\}} \sum_{t=0}^{\infty} \beta^{t}\left[\log \Theta_{t}+(1-\alpha) \log S_{t}-\frac{1}{1+\phi} \frac{1}{M\left(1+\tau^{L}\right)} \Theta_{t}^{-1}\left[(1-\alpha) \Theta_{t}+\alpha \Lambda_{H, t}\right]\right]
$$

subject to

$$
\begin{aligned}
N F A_{0} & =\alpha \sum_{t=0}^{\infty} \beta^{t}\left(\Lambda_{H, t}-\Theta_{t}\right), \\
1 & =M\left(1+\tau^{L}\right) \frac{1}{A_{H, t}^{1+\phi}} S_{t}^{1+\phi} C_{t}^{* \phi+1} \Theta_{t}\left[(1-\alpha) \Theta_{t}+\alpha \Lambda_{H, t}\right]^{\phi} .
\end{aligned}
$$

Solving for $S_{t}$ as a function of $\Theta_{t}$ from the second constraint and substituting we can rewrite this problem as a function of the path for $\left\{\Theta_{t}\right\}$. Then the first order condition is then

$$
\frac{\phi+\alpha}{1+\phi}-\frac{(1-\alpha)^{2} \phi}{1+\phi} \frac{\Theta_{t}}{\alpha \Lambda_{H, t}+(1-\alpha) \Theta_{t}}+\frac{(1-\alpha) \alpha \Lambda_{H, t}}{1+\phi} \frac{1}{\Theta_{t}}-\Gamma \Theta_{t}=0
$$

For a given value of $\Gamma$, then $\Theta_{t}$ is independent of $A_{H, t}$ and increasing in $\Lambda_{H, t}$. Since $\frac{\Theta_{t+1}}{\Theta_{t}}=\left(1+\tau_{t}\right)^{\frac{1}{\sigma}}$ the result follows.

\section{A.3 Proof of Proposition 4}

We start with transitory productivity shocks. The optimal allocation is constant for $t \geq 1$. Let $\Theta$ be the corresponding value of $\Theta$, which is related to $\Theta_{0}$ by $(\Theta-1)=\frac{1-\beta}{\beta}\left(1-\Theta_{0}\right)$. This immediately implies that $\tau_{t}=0$ for $t \geq 1$. Then we need to solve the $t=0$ problem $\max _{\Theta_{0}} U\left(\Theta_{0}, A_{0}\right)$ where

$$
U\left(\Theta_{0}, A_{0}\right)=C^{* 1-\sigma} \frac{\Theta_{0}^{1-\sigma}}{1-\sigma}-\frac{C^{* 1+\phi}}{A_{0}^{1+\phi}} \frac{\left[(1-\alpha) \Theta_{0}+\alpha\right]^{1+\phi}}{1+\phi}+\beta V\left(\frac{1}{\beta} C^{* 1-\sigma} \alpha\left(1-\Theta_{0}\right)\right) .
$$

It is easily verified that $U_{A_{0}, \Theta_{0}}>0$. This implies that $\Theta_{0}$ is an increasing function of $A_{0}$. This in turn implies that $\Theta$ is a decreasing function of $A_{0}$. The first result in the proposition follows since capital controls $\tau_{0}$ are given by $\frac{\Theta}{\Theta_{0}}=\left(1+\tau_{0}\right)^{\frac{1}{\sigma}}$. 
Now consider temporary export demand shocks. The optimal allocation is constant allocation for $t \geq 1$, so we drop the $t$ subscripts. This immediately implies that $\tau_{t}=$ 0 for $t \geq 1$. We are interested in the limit of small time intervals $(\beta \rightarrow 1)$. We have $\frac{1-\beta}{\beta} \alpha\left(\Lambda_{H, 0}-\Theta_{0}\right)=\alpha\left(\mathcal{Q}^{\frac{1}{\sigma}-\eta} \Theta-S^{\gamma-1} \Lambda_{H}\right)$. Using the other constraints, we can write the right hand side as a function $J(\Theta)$ with $J(1)=0$. We find that to a first order in $1-\beta$ we can write

$$
\Theta=1+\frac{1}{J^{\prime}(1)}(1-\beta) \alpha\left(\Lambda_{H, 0}-\Theta_{0}\right)+O(1-\beta)^{2} .
$$

We can then write welfare for $t \geq 1$ as a function $H(\Theta)$. We find that up to second order terms in $1-\beta$, we have to solve $\max _{\Theta_{0}} U\left(\Theta_{0}, \Lambda_{0}\right)$ where

$$
U\left(\Theta_{0}, \Lambda_{H, 0}\right)=C^{* 1-\sigma} \frac{\Theta_{0}^{1-\sigma}}{1-\sigma}-\frac{C^{* 1+\phi}}{A^{1+\phi}} \frac{\left[(1-\alpha) \Theta_{0}+\alpha \Lambda_{H, 0}\right]^{1+\phi}}{1+\phi}+\frac{H^{\prime}(1)}{J^{\prime}(1)} \alpha\left(\Lambda_{H, 0}-\Theta_{0}\right) .
$$

It is easily verified that $U_{\Lambda_{H, 0}, \Theta_{0}}<0$. This implies that $\Theta_{0}$ is a decreasing function of $\Lambda_{H, 0}$. Moreover, up to first order terms in $1-\beta, \Theta$ is constant. The second result in the proposition follows since capital controls $\tau_{0}$ are given by $\frac{\Theta}{\Theta_{0}}=\left(1+\tau_{0}\right)^{\frac{1}{\sigma}}$.

Finally consider foreign consumption shocks. The optimal allocation is constant for $t \geq 1$. Let $\Theta$ be the corresponding value of $\Theta$, which is related to $\Theta_{0}$ by $C^{* 1-\sigma}(\Theta-1)=$ $\frac{1-\beta}{\beta} C_{0}^{* 1-\sigma}\left(1-\Theta_{0}\right)$. This immediately implies that $\tau_{t}=0$ for $t \geq 1$. We have to solve $\max _{\Theta_{0}} U\left(\Theta_{0}, \Lambda_{0}\right)$ where

$$
U\left(\Theta_{0}, C_{0}^{*}\right)=C_{0}^{* 1-\sigma} \frac{\Theta_{0}^{1-\sigma}}{1-\sigma}-\frac{C_{0}^{* 1+\phi}}{A^{1+\phi}} \frac{\left[(1-\alpha) \Theta_{0}+\alpha\right]^{1+\phi}}{1+\phi}+\beta V\left(\frac{1}{\beta} C_{0}^{* 1-\sigma} \alpha\left(1-\Theta_{0}\right)\right) .
$$

We find

$$
\begin{aligned}
U_{C_{0}^{*}, \Theta_{0}}\left(\Theta_{0}, C_{0}^{*}\right)=(1-\sigma) & C_{0}^{*-\sigma} \Theta_{0}^{-\sigma}-(1-\alpha)(1+\phi) \frac{C_{0}^{* \phi}}{A^{1+\phi}}\left[(1-\alpha) \Theta_{0}+\alpha\right]^{\phi} \\
- & (1-\sigma) C_{0}^{*-\sigma} \alpha V^{\prime}\left(\frac{1}{\beta} C_{0}^{* 1-\sigma} \alpha\left(1-\Theta_{0}\right)\right) \\
& -(1-\sigma) C_{0}^{* 1-\sigma} C_{0}^{* 1-\sigma}\left(1-\Theta_{0}\right) \frac{1}{\beta} \alpha^{2} V^{\prime \prime}\left(\frac{1}{\beta} C_{0}^{* 1-\sigma} \alpha\left(1-\Theta_{0}\right)\right) .
\end{aligned}
$$

Clearly, for $\sigma=1$, we have $U_{C_{0}^{*}, \Theta_{0}}\left(\Theta_{0}, C_{0}^{*}\right)<0$. The third result follows. 


\section{A.4 Proof of Proposition 5}

In the Cole-Obstfeld case, we can write $V\left(N F A_{1}\right)$ as the maximization over $\left\{\Theta_{t}\right\}_{t \geq 1}$ of

$$
\begin{aligned}
(1-\alpha) \log \left(A_{H, t}\right)+\alpha \log C_{t}^{*} & +\frac{1-\alpha}{1+\phi} \log (1-\alpha)+\frac{\phi+\alpha}{1+\phi} \log \left(\Theta_{t}\right) \\
- & \frac{(1-\alpha) \phi}{1+\phi} \log \left[\alpha \Lambda_{H, t}+(1-\alpha) \Theta_{t}\right]-\frac{(1-\alpha) \frac{\alpha \Lambda_{H, t}+(1-\alpha) \Theta_{t}}{\Theta_{t}}}{1+\phi}
\end{aligned}
$$

subject to

$$
N F A_{1}=-\sum_{t=0}^{\infty} \beta^{t} \alpha\left(\Lambda_{H, t}-\Theta_{t}\right)
$$

Consider first productivity shocks. For all $t \geq 1$ productivity $A_{H, t}$, the solution of the planning problem for $t \geq 1$ features a constant allocation, for which we drop the $t$ subscripts. This immediately implies that optimal capital controls are zero for $t \geq 1$. It is immediate that the optimal $\Theta$ is independent of the path $A_{H, t}$ for $t \geq 1$. The result for productivity shocks then simply follows from Proposition 4.

Consider next foreign consumption shocks. For all $t \geq 1$ productivity $C_{t}^{*}$, the solution of the planning problem for $t \geq 1$ features a constant allocation, for which we drop the $t$ subscripts. This immediately implies that optimal capital controls are zero for $t \geq 1$. It is immediate that the optimal $\Theta$ is independent of the path $C_{t}^{*}$ for $t \geq 1$. The result for productivity shocks then simply follows from Proposition 4.

Consider next permanent export demand shocks $\Lambda_{H, t}=\Lambda_{H}$ for all $t \geq 0$. For all $t \geq 1$ the solution of the planning problem for $t \geq 1$ features a constant allocation, for which we drop the $t$ subscripts. This immediately implies that optimal capital controls are zero for $t \geq 1$. We also find it convenient to define $\tilde{\Theta}_{0}=\Theta_{0}-\Lambda_{H}$ and $\tilde{\Theta}=\Theta-\Lambda_{H}$. The country budget constraint establishes the following relationship between $\tilde{\Theta}$ and $\tilde{\Theta}_{0}$ : $\tilde{\Theta}=-\frac{1-\beta}{\beta} \tilde{\Theta}_{0}$. We are left with the following maximization problem

$$
\max \left[\log \left[\Lambda_{0}+\tilde{\Theta}_{0}\right]+\log C^{*}-\frac{1}{1+\phi}\left(\frac{C^{*}}{A_{H}}\right)^{1+\phi}\left(\Lambda_{H}+(1-\alpha) \tilde{\Theta}_{0}\right)^{1+\phi}+\beta V\left(-\frac{\alpha}{\beta} \tilde{\Theta}_{0}\right)\right]
$$

where

$$
\begin{aligned}
(1-\beta) V(\tilde{\Theta})=(1-\alpha) \log \left(A_{H}\right) & +\alpha \log C^{*}+\frac{1-\alpha}{1+\phi} \log (1-\alpha)+\frac{\phi+\alpha}{1+\phi} \log \left[\tilde{\Theta}+\Lambda_{H}\right] \\
& -\frac{(1-\alpha) \phi}{1+\phi} \log \left[\Lambda_{H}+(1-\alpha) \tilde{\Theta}\right]-\frac{(1-\alpha) \frac{\Lambda_{H}+(1-\alpha) \tilde{\Theta}}{\Lambda+\tilde{\Theta}}}{1+\phi}
\end{aligned}
$$


Let $U\left(\tilde{\Theta}_{0}, \Lambda_{H}\right)$ be the objective function. We can compute

$$
\begin{aligned}
U_{\tilde{\Theta}_{0}, \Lambda_{H}}= & \frac{\phi+\alpha}{1+\phi} \frac{1}{\left(\tilde{\Theta}+\Lambda_{H}\right)^{2}}+\frac{\alpha(1-\alpha)}{1+\phi} \frac{\tilde{\Theta}-\Lambda_{H}}{\left(\Lambda_{H}+\tilde{\Theta}\right)^{3}}-\frac{1}{\left(\Lambda_{H}+\tilde{\Theta}_{0}\right)^{2}} \\
& -(1-\alpha) \phi\left(\frac{C^{*}}{A_{H}}\right)^{1+\phi}\left(\Lambda_{H}+(1-\alpha) \tilde{\Theta}_{0}\right)^{\phi-1}-\frac{(1-\alpha)^{2} \phi}{1+\phi} \frac{1}{\left[\Lambda_{H}+(1-\alpha) \tilde{\Theta}\right]^{2}}
\end{aligned}
$$

For $\tilde{\Theta}_{0}=0$ (and hence $\tilde{\Theta}=0$ ) we always have $U_{\tilde{\Theta}_{0}, \Lambda_{H}}\left(0, \Lambda_{H}\right)<0$, so that at least for small shocks, $\tilde{\Theta}_{0}$ is decreasing in $\Lambda_{H}$ and $\tilde{\Theta}$ is increasing in $\Lambda_{H}$ with $\tilde{\Theta}=\tilde{\Theta}_{0}=0$ for $\Lambda_{H}=1$. The result in the proposition follows since capital controls $\tau_{0}$ are given by $\frac{\Lambda_{H}+\tilde{\Theta}}{\Lambda_{H}+\tilde{\Theta}_{0}}=1+\tau_{0}$.

Finally, let's us consider wealth shocks $N F A_{0} \neq 0$. For all $t \geq 1$ the solution of the planning problem for $t \geq 1$ features a constant allocation, for which we drop the $t$ subscripts. This immediately implies that optimal capital controls are zero for $t \geq 1$. We also find it convenient to define $\tilde{\Theta}_{0}=\Theta_{0}-1-(1-\beta) \frac{1}{\alpha} N F A_{0}$ and $\tilde{\Theta}=\Theta-1-(1-\beta) \frac{1}{\alpha} N F A_{0}$. The country budget constraint establishes the following relationship between $\tilde{\Theta}$ and $\tilde{\Theta}_{0}$ : $\tilde{\Theta}=-\frac{1-\beta}{\beta} \tilde{\Theta}_{0}$. We are left with the following maximization problem over $\tilde{\Theta}_{0}$ :

$$
\begin{aligned}
\log \left(\tilde{\Theta}_{0}+1\right. & \left.+(1-\beta) \frac{1}{\alpha} N F A_{0}\right)+\log C^{*} \\
& -\frac{1}{1+\phi}\left(\frac{C^{*}}{A_{H}}\right)^{1+\phi}\left((1-\alpha) \tilde{\Theta}_{0}+(1-\beta) \frac{1-\alpha}{\alpha} N F A_{0}+1\right)^{1+\phi}+\beta V(\tilde{\Theta})
\end{aligned}
$$

where

$$
\begin{aligned}
(1-\beta) V(\tilde{\Theta})=(1-\alpha) \log \left(A_{H}\right)+\alpha \log C^{*}+\frac{1-\alpha}{1+\phi} \log (1-\alpha) & \\
+\frac{\phi+\alpha}{1+\phi} \log \left[\tilde{\Theta}+1+(1-\beta) \frac{1}{\alpha} N F A_{0}\right]-\frac{(1-\alpha) \phi}{1+\phi} \log [ & \left.(1-\alpha) \tilde{\Theta}+(1-\beta) \frac{1-\alpha}{\alpha} N F A_{0}+1\right] \\
- & -\frac{(1-\alpha) \frac{(1-\alpha) \tilde{\Theta}+(1-\beta) \frac{1-\alpha}{\alpha} N F A_{0}+1}{\tilde{\Theta}+1+(1-\beta) \frac{1}{\alpha} N F A_{0}}}{1+\phi} .
\end{aligned}
$$


Let $U\left(\tilde{\Theta}_{0}, N F A_{0}\right)$ be the objective function. We have

$$
\begin{gathered}
\frac{1}{(1-\beta) \frac{1}{\alpha}} U_{\tilde{\Theta}_{0}, N F A_{0}}=\frac{\phi+\alpha}{1+\phi} \frac{1}{\left[\tilde{\Theta}+1+(1-\beta) \frac{1}{\alpha} N F A_{0}\right]^{2}}-\frac{1}{\left[\tilde{\Theta}_{0}+1+(1-\beta) \frac{1}{\alpha} N F A_{0}\right]^{2}} \\
-(1-\alpha)^{2} \phi\left(\frac{C^{*}}{A_{H}}\right)^{1+\phi}\left((1-\alpha) \tilde{\Theta}_{0}+(1-\beta) \frac{1-\alpha}{\alpha} N F A_{0}+1\right)^{\phi-1} \\
-\frac{(1-\alpha)^{3} \phi}{1+\phi} \frac{1}{\left[(1-\alpha) \tilde{\Theta}+(1-\beta) \frac{1-\alpha}{\alpha} N F A_{0}+1\right]^{2}}-2 \frac{\alpha(1-\alpha)}{1+\phi} \frac{1}{\left[\tilde{\Theta}+1+(1-\beta) \frac{1}{\alpha} N F A_{0}\right]^{3}}
\end{gathered}
$$

For $\tilde{\Theta}_{0}=0$ (and hence $\tilde{\Theta}=0$ ) we always have $U_{\tilde{\Theta}_{0}, N F A_{0}}\left(0, N F A_{0}\right)<0$, so that at least for small shocks, so that at least for small shocks, $\tilde{\Theta}_{0}$ is decreasing in $W_{0}$ and $\tilde{\Theta}$ is increasing in $W_{0}$ with $\tilde{\Theta}=\tilde{\Theta}_{0}=0$ for $\Lambda_{H}=1$. The result in the proposition follows since capital controls $\tau_{0}$ are given by $\frac{1+(1-\beta) \frac{1}{\alpha} N F A_{0}+\tilde{\Theta}}{1+(1-\beta) \frac{1}{\alpha} N F A_{0}+\tilde{\Theta}_{0}}=1+\tau_{0}$. . More generally we have $U_{\tilde{\Theta}_{0}, N F A_{0}}\left(\tilde{\Theta}_{0}, N F A_{0}\right)<0$ for all $\tilde{\Theta}_{0}<0$ which implies, together with the concavity of $U$ in $\tilde{\Theta}_{0}$, that for positive shocks to $N F A_{0}, \tilde{\Theta}_{0}$ is a decreasing function of $N F A_{0}$ so that the result generalizes to any positive shock to initial wealth $N F A_{0}>0$.

\section{A.5 Proof or Proposition 6}

We begin with the latter three claims in the proposition then return to the first claim.

We note that in general, for rigid prices or for prices set one period in advance, the planning problem with capital controls for $\alpha>0$ can be written in a way that makes it continuous in $\alpha$. Then this shows that the unique solution of the planning problem for $\alpha>$ 0 converges to the unique solution of a limit planning problem when $\alpha \rightarrow 0$. This limit planning problem is more constrained than the planning problem of a closed economy $(\alpha=0)$ : they coincide except that in the latter, the country budget constraint is dropped. The solution of the closed economy planning problem is the flexible price allocation which coincides with first best. Hence the optimal allocation with capital controls converges to the first best allocation as $\alpha \rightarrow 0$ if and only if the country budget constraint is not binding in the limit planning problem. We illustrate this logic in detail for the second claim and in less detail for the other claims to avoid repetition.

We start by the case of rigid prices with capital controls. The planning problem for $\alpha>0$ can be rewritten as

$$
\max _{\left\{\Theta_{t}\right\}} \sum_{t=0}^{\infty} \beta^{t}\left[\frac{1}{1-\sigma} \Theta_{t}^{1-\sigma} C_{t}^{* 1-\sigma}-\frac{1}{1+\phi}\left(\alpha \Lambda_{H, t}+\Theta_{t}(1-\alpha)\right)^{1+\phi}\left(\frac{C_{t}^{*}}{A_{H, t}}\right)^{1+\phi}\right]
$$


subject to,

$$
\sum_{t=0}^{\infty} \beta^{t} C_{t}^{* 1-\sigma}\left[\Theta_{t}-\Lambda_{H, t}\right]=0
$$

It is continuous in $\alpha$ and admits a unique solution since the problem is convex. This shows that the optimal allocation with capital controls as $\alpha \rightarrow 0$ converges to the solution of the following limit planning problem:

$$
\max _{\left\{\Theta_{t}\right\}} \sum_{t=0}^{\infty} \beta^{t}\left[\frac{1}{1-\sigma} \Theta_{t}^{1-\sigma} C_{t}^{* 1-\sigma}-\frac{1}{1+\phi} \Theta_{t}^{1+\phi}\left(\frac{C_{t}^{*}}{A_{H, t}}\right)^{1+\phi}\right]
$$

subject to,

$$
\sum_{t=0}^{\infty} \beta^{t} C_{t}^{* 1-\sigma}\left[\Lambda_{H, t}-\Theta_{t}\right]=0
$$

For generic shocks to the sequences $\left\{A_{H, t}, \Lambda_{H, t}, C_{t}^{*}\right\}_{t \geq 0}$, this limit planning problem is strictly more constrained than the corresponding closed economy planning problem which coincides with the planning problem (33) but where the budget constraint is dropped. In the closed economy, capital controls act as a perfect substitute to monetary policy and allow to replicate the flexible-price allocation, which coincides with the first best allocation. The claim that the budget constraint (34) is binding in the limit program (33) can be seen as follows. Let $C_{t}$ be the flexible price allocation of the closed economy and let $\tilde{\Theta}_{t}=\frac{C_{t}}{C_{t}^{*}}$. Then unless

$$
\sum_{t=0}^{\infty} \beta^{t} C_{t}^{* 1-\sigma}\left[\Lambda_{H, t}-\tilde{\Theta}_{t}\right]=0,
$$

which does not hold for generic shocks, the budget constraint is binding.

Next consider the third claim. For risk premium shocks $\left\{\Psi_{t}\right\}_{t \geq 0}$, the flexible price allocation of the closed economy features $C_{t}=C$ so that $\tilde{\Theta}_{t}=\Lambda_{H, t}$ since $C=\Lambda_{H, t} C_{t}^{*}$. It is then immediate that in this case,

$$
\sum_{t=0}^{\infty} \beta^{t} C_{t}^{* 1-\sigma}\left[\Lambda_{H, t}-\tilde{\Theta}_{t}\right]=0
$$

which shows that the budget constraint is not binding in the limit planning problem.

We now show that with one period in advance price setting with capital controls the allocation converges to the flexible price allocation of the closed economy. It suffices to show that the budget constraint is not binding in the limit $\alpha \rightarrow 0$. To see that this is the case, rewrite limit of the budget constraints as (using the fact that in the limit $\alpha \rightarrow 0$, 
$\left.\mathcal{Q}_{t}=S_{t}\right)$

$$
C_{0}^{* 1-\sigma}\left[\Lambda_{H, 0}-\Theta_{0}\right]+\sum_{t=1}^{\infty} \beta^{t} C_{t}^{* 1-\sigma} \mathcal{Q}_{t}^{-\eta}\left[\mathcal{Q}_{t}^{\eta+\gamma-1} \Lambda_{H, t}-\mathcal{Q}_{t}^{\frac{1}{\sigma}} \Theta_{t}\right]=0
$$

Now note that for $t \geq 1$, when $\alpha \rightarrow 0$, welfare and the equilibrium conditions (9), (10), (11), (12) with $\Delta_{t}=1,(16)$, depend on $\Theta_{t}$ and $\mathcal{Q}_{t}$ only through $\tilde{\Theta}_{t}=\Theta_{t} \mathcal{Q}_{t}^{\frac{1}{\sigma}}$. It then suffices to let choose $\tilde{\Theta}_{t}=\frac{C_{t}}{C_{t}^{*}}$ where $C_{t}$ is the flexible price allocation of the closed economy, and to pick a sequence $\left\{\mathcal{Q}_{t}\right\}_{t \geq 1}$ (there are infinitely many such sequences) such that

$$
C_{0}^{* 1-\sigma}\left[\Lambda_{H, 0}-\tilde{\Theta}_{0}\right]+\sum_{t=1}^{\infty} \beta^{t} C_{t}^{* 1-\sigma} \mathcal{Q}_{t}^{-\eta}\left[\mathcal{Q}_{t}^{\eta+\gamma-1} \Lambda_{H, t}-\tilde{\Theta}_{t}\right]=0,
$$

and to then pick $\Theta_{t}=\frac{\tilde{\Theta}_{t}}{\mathcal{Q}_{t}^{\frac{1}{\sigma}}}$ and $\Theta_{0}=\tilde{\Theta}_{0}$. This immediately shows that the budget constraint is not binding in the limit $\alpha \rightarrow 0$.

We now return to the first claim. The result in the case of rigid prices follows directly from the second claim, which we already proved. It remains to prove the result where prices are set one period in advance. To see this, and following the proof of the fourth claim, it suffices to show that among the sequences $\left\{\mathcal{Q}_{t}\right\}_{t \geq 1}$ such that

$$
C_{0}^{* 1-\sigma}\left[\Lambda_{H, 0}-\tilde{\Theta}_{0}\right]+\sum_{t=1}^{\infty} \beta^{t} C_{t}^{* 1-\sigma} \mathcal{Q}_{t}^{-\eta}\left[\mathcal{Q}_{t}^{\eta+\gamma-1} \Lambda_{H, t}-\tilde{\Theta}_{t}\right]=0
$$

none satisfies $\frac{\tilde{\Theta}_{t}}{\mathcal{Q}_{t}^{\frac{1}{T}}}=\tilde{\Theta}_{0}$, which is clearly true unless

$$
C_{0}^{* 1-\sigma}\left[\Lambda_{H, 0}-\tilde{\Theta}_{0}\right]+\sum_{t=1}^{\infty} \beta^{t} C_{t}^{* 1-\sigma}\left(\frac{\tilde{\Theta}_{t}}{\tilde{\Theta}_{0}}\right)^{-\eta}\left[\left(\frac{\tilde{\Theta}_{t}}{\tilde{\Theta}_{0}}\right)^{\eta+\gamma-1} \Lambda_{H, t}-\tilde{\Theta}_{t}\right]=0,
$$

which does not hold for generic shocks.

\section{A.6 Derivation of the Loss Function in Section 4}

We focus on Cole-Obstfeld case $\sigma=\gamma=\eta=1$. We provide an derivation that spans Section 4.2 in which case $\bar{\theta}_{t}=\bar{\theta}$ and Section 4.3 in which case $\bar{\theta}_{t}=\int_{0}^{t} \psi_{s} d s-\int_{0}^{\infty} \psi_{t} e^{-\rho t} d t$.

We have the exact relationship

$$
c_{t}=\theta_{t}+c_{t}^{*}+(1-\alpha) s_{t}
$$


and the following second order approximation

$$
y_{t}=c_{t}^{*}+s_{t}+(1-\alpha) \theta_{t}+\alpha \lambda_{h, t}+\frac{1}{2} \alpha(1-\alpha) \lambda_{h, t}^{2}+\frac{1}{2} \alpha(1-\alpha) \theta_{t}^{2}-\alpha(1-\alpha) \theta_{t} \lambda_{h, t} .
$$

Using these equations, we can derive

$$
\begin{aligned}
c_{t}=\alpha c_{t}^{*}+\theta_{t} \alpha(2- & \alpha)+(1-\alpha) y_{t} \\
& +(1-\alpha)\left[-\alpha \lambda_{h, t}-\frac{1}{2} \alpha(1-\alpha) \lambda_{h, t}^{2}\right]+\frac{1}{2}(1-\alpha)^{2}\left[-\alpha \theta_{t}^{2}+2 \alpha \theta_{t} \lambda_{h, t}\right] .
\end{aligned}
$$

Hence in gaps,

$$
\hat{c}_{t}=(1-\alpha) \hat{y}_{t}+\alpha(2-\alpha) \hat{\theta}_{t}+\frac{1}{2}(1-\alpha)^{2}\left[-\alpha \hat{\theta}_{t}\left(\hat{\theta}_{t}+2 \bar{\theta}_{t}\right)+2 \alpha \hat{\theta}_{t} \lambda_{h, t}\right] .
$$

We can use this expression to derive

$$
\begin{aligned}
\log C_{t} & =\bar{c}_{t}+\hat{c}_{t} \\
& =\bar{c}_{t}+(1-\alpha) \hat{y}_{t}+\alpha(2-\alpha) \hat{\theta}_{t}+\frac{1}{2}(1-\alpha)^{2}\left[-\alpha\left(\hat{\theta}_{t}+\bar{\theta}_{t}\right)^{2}+2 \alpha\left(\hat{\theta}_{t}+\bar{\theta}_{t}\right) \lambda_{h, t}\right] .
\end{aligned}
$$

We have

$$
\frac{N_{t}^{1+\phi}}{1+\phi}=\frac{\bar{N}_{t}^{1+\phi}}{1+\phi}+\bar{N}_{t}^{1+\phi}\left[\hat{y}_{t}+z_{t}+\frac{1}{2}(1+\phi) \hat{y}_{t}^{2}\right]
$$

where

$$
z_{t}=\log \int\left(\frac{P_{H, t}(j)}{P_{H, t}}\right)^{-\epsilon} \approx \frac{\epsilon}{2} \sigma_{p_{H, t}}^{2} .
$$

Using the fact that $\bar{N}_{t}^{1+\phi}=1-\alpha$ for all $t$, we get the following expression for the objective function:

$$
\begin{aligned}
& \int_{0}^{\infty} e^{-\rho t}\left(\frac{U_{t}-\bar{U}_{t}}{C U_{c}}\right) d t= \\
& -\frac{(1-\alpha)(1+\phi)}{2} \int_{0}^{\infty} e^{-\rho t}\left[\alpha_{\pi} \pi_{H, t}^{2}+\hat{y}_{t}^{2}-\frac{2 \alpha(2-\alpha)}{(1-\alpha)(1+\phi)} \hat{\theta}_{t}-\frac{1-\alpha}{1+\phi}\left[-\alpha \hat{\theta}_{t}\left(\hat{\theta}_{t}+2 \bar{\theta}_{t}\right)+2 \alpha \hat{\theta}_{t} \lambda_{h, t}\right]\right] d t,
\end{aligned}
$$

where $\alpha_{\pi}=\epsilon /[\lambda(1+\phi)]$.

We now use a second order approximation of the country budget constraint to replace the linear term in $\hat{\theta}_{t}$ in the expression above. We find that a second order approximation for $n x_{t}$ :

$$
n x_{t}=\alpha\left(\theta_{t}-\lambda_{t}+\frac{1}{2} \theta_{t}^{2}-\frac{1}{2} \lambda_{t}^{2}\right) .
$$


We first abstract from risk premium shocks. We then have the following second order approximation for the budget constraint

$$
\alpha \int_{0}^{\infty} e^{-\rho t}\left(\hat{\theta}_{t}+\frac{1}{2} \hat{\theta}_{t}\left(\hat{\theta}_{t}+2 \bar{\theta}_{t}\right)\right)=0
$$

so that we can replace the linear term in $\hat{\theta}_{t}$ in the expression for welfare to get the following expression for the loss function:

$$
(1-\alpha)(1+\phi) \int e^{-\rho t}\left[\frac{1}{2} \alpha_{\pi} \pi_{H, t}^{2}+\frac{1}{2} \hat{y}_{t}^{2}+\frac{1}{2} \alpha_{\theta} \hat{\theta}_{t}\left(\hat{\theta}_{t}+2 \bar{\theta}_{t}-2 \alpha_{\psi} \lambda_{h, t}\right)\right] d t,
$$

or up to a constant

$$
(1-\alpha)(1+\phi) \int e^{-\rho t}\left[\frac{1}{2} \alpha_{\pi} \pi_{H, t}^{2}+\frac{1}{2} \hat{y}_{t}^{2}+\frac{1}{2} \alpha_{\theta}\left(\hat{\theta}_{t}+\bar{\theta}_{t}-\alpha_{\psi} \lambda_{h, t}\right)^{2}\right] d t
$$

where

$$
\alpha_{\psi}=\frac{1-\alpha}{\frac{2-\alpha}{1-\alpha}+1-\alpha} \quad \text { and } \quad \alpha_{\theta}=\frac{\alpha}{1+\phi}\left(\frac{2-\alpha}{1-\alpha}+1-\alpha\right) .
$$

We now focus on the case of a pure risk premium shock. A first order approximation of the discount factor $e^{-\rho t} e^{-\int_{0}^{t} \psi_{t} d t}$ is $e^{-\rho t}\left[1+\bar{\theta}_{0}-\bar{\theta}_{t}\right]$. Combining the two, we get the following second order approximation for the budget constraint

$$
\alpha \int_{0}^{\infty} e^{-\rho t}\left(\hat{\theta}_{t}+\frac{1}{2} \hat{\theta}_{t}\left(\hat{\theta}_{t}+2 \bar{\theta}_{t}\right)+\left(\bar{\theta}_{0}-\bar{\theta}_{t}\right) \hat{\theta}_{t}\right)=0,
$$

so that we can replace the linear term in $\hat{\theta}_{t}$ in the expression for welfare to get the following expression for the loss function:

$$
(1-\alpha)(1+\phi) \int e^{-\rho t}\left[\frac{1}{2} \alpha_{\pi} \pi_{H, t}^{2}+\frac{1}{2} \hat{y}_{t}^{2}+\frac{1}{2} \alpha_{\theta} \hat{\theta}_{t}\left(\hat{\theta}_{t}+2\left(\alpha_{\psi} \bar{\theta}_{t}+\left(1-\alpha_{\psi}\right) \bar{\theta}_{0}\right)\right)\right] d t,
$$

or up to a constant

$$
(1-\alpha)(1+\phi) \int e^{-\rho t}\left[\frac{1}{2} \alpha_{\pi} \pi_{H, t}^{2}+\frac{1}{2} \hat{y}_{t}^{2}+\frac{1}{2} \alpha_{\theta}\left(\hat{\theta}_{t}+\alpha_{\psi} \bar{\theta}_{t}+\left(1-\alpha_{\psi}\right) \bar{\theta}_{0}\right)^{2}\right] d t .
$$




\section{A.7 FOCs for the Optimal Control Problem in Section 4.2}

We can incorporate a multiplier $\Gamma$ on the intertemporal budget constraint. We are left with the following optimal control problem

$$
\min _{\left\{\pi_{H, t}, \hat{y}_{t}, i_{t}, \hat{\theta}_{t}\right\}} \int_{0}^{\infty} e^{-\rho t}\left[\frac{1}{2} \alpha_{\pi} \pi_{H, t}^{2}+\frac{1}{2} \hat{y}_{t}^{2}+\alpha_{\theta} \frac{1}{2}\left(\hat{\theta}_{t}+\bar{\theta}-\alpha_{\psi} \lambda_{t}\right)^{2}+\Gamma \hat{\theta}_{t}\right] d t
$$

subject to

$$
\begin{gathered}
\dot{\pi}_{H, t}=\rho \pi_{H, t}-\hat{\kappa} \hat{y}_{t}-\lambda \alpha \hat{\theta}_{t}, \\
\dot{\hat{y}}_{t}=(1-\alpha)\left(i_{t}-i_{t}^{*}\right)-\pi_{H, t}+i_{t}^{*}-\bar{r}_{t}, \\
\dot{\hat{\theta}}_{t}=i_{t}-i_{t}^{*}, \\
\hat{y}_{0}=(1-\alpha) \hat{\theta}_{0}-\bar{s}_{0} .
\end{gathered}
$$

Let $\mu_{\pi, t}, \mu_{y, t}$ and $\mu_{\theta, t}$ be the costates. We find the following first-order conditions

$$
\begin{gathered}
\dot{\mu}_{\pi, t}=-\alpha_{\pi} \pi_{H, t}+\mu_{y, t} \\
\dot{\mu}_{y, t}=-\hat{y}_{t}+\mu_{\pi, t} \hat{\kappa}+\rho \mu_{y, t}, \\
\dot{\mu}_{\theta, t}=-\alpha_{\theta}\left(\hat{\theta}_{t}+\bar{\theta}-\alpha_{\psi} \lambda_{h, t}\right)-\Gamma+\mu_{\pi, t} \lambda \alpha+\rho \mu_{\theta, t}, \\
(1-\alpha) \mu_{y, t}+\mu_{\theta, t}=0, \\
\mu_{\pi, 0}=0, \\
\mu_{\theta, 0}=-(1-\alpha) \mu_{y, 0},
\end{gathered}
$$

together with the transversality conditions $\lim _{t \rightarrow \infty} e^{-\rho t} \mu_{y, t} \hat{y}_{t}=0, \lim _{t \rightarrow \infty} e^{-\rho t} \mu_{\theta, t} \hat{\theta}_{t}=0$, and $\lim _{t \rightarrow \infty} e^{-\rho t} \mu_{\pi, t} \hat{\pi}_{t}=0$.

\section{A.8 Proof of Proposition 7}

We have $\hat{y}_{t}=-\frac{\alpha}{1+\phi} \hat{\theta}_{t}$ and the planning problem is

$$
\min \int e^{-\rho t}\left[\frac{1}{2}\left(\frac{\alpha}{1+\phi}\right)^{2}\left(\hat{\theta}_{t}\right)^{2}+\alpha_{\theta} \frac{1}{2}\left(\hat{\theta}_{t}+\bar{\theta}-\alpha_{\psi} \lambda_{h, t}\right)^{2}\right] d t
$$

s.t.

$$
\int e^{-\rho t} \hat{\theta}_{t} d t=0
$$


The solution is

$$
\left[\left(\frac{\alpha}{1+\phi}\right)^{2}+\alpha_{\theta}\right] \hat{\theta}_{t}=-\alpha_{\theta} \bar{\theta}+\alpha_{\theta} \alpha_{\psi} \lambda_{h, t}-\Gamma
$$

Since $\int_{0}^{\infty} e^{-\rho t} \bar{\theta} d t=\frac{1}{\alpha} N F A_{0}+\int_{0}^{\infty} e^{-\rho t} \lambda_{h, t} d t$ we find

$$
\begin{gathered}
\hat{\theta}_{t}=\frac{\alpha_{\theta} \alpha_{\psi}}{\left(\frac{\alpha}{1+\phi}\right)^{2}+\alpha_{\theta}}\left[\lambda_{h, t}-\rho \int_{0}^{\infty} \lambda_{h, t} e^{-\rho t} d t\right], \\
\hat{y}_{t}=-\frac{\alpha}{1+\phi} \frac{\alpha_{\theta} \alpha_{\psi}}{\left(\frac{\alpha}{1+\phi}\right)^{2}+\alpha_{\theta}}\left[\lambda_{h, t}-\rho \int_{0}^{\infty} \lambda_{h, t} e^{-\rho t} d t\right] .
\end{gathered}
$$

\section{A.9 Proof of Proposition 8}

We are led to the following planning problem:

$$
\min \int e^{-\rho t}\left[\frac{1}{2} \hat{y}_{t}^{2}+\alpha_{\theta} \frac{1}{2}\left(\hat{\theta}_{t}+\bar{\theta}-\alpha_{\psi} \lambda_{h, t}\right)^{2}\right] d t
$$

s.t.

$$
\begin{gathered}
\dot{\hat{y}}_{t}=(1-\alpha)\left(i_{t}-i_{t}^{*}\right)+i_{t}^{*}-\bar{r}_{t} \\
\dot{\hat{\theta}}_{t}=i_{t}-i_{t}^{*}, \\
\int \hat{\theta}_{t} e^{-\rho t} d t=0, \\
\hat{y}_{0}=(1-\alpha) \hat{\theta}_{0}-\bar{s}_{0} .
\end{gathered}
$$

Let $\Gamma$ be the multiplier on the budget constraint, and let $\mu_{x, t}$ and $\mu_{\theta, t}$ be the co-states. We find the following first-order conditions

$$
\begin{gathered}
\dot{\mu}_{y, t}=-\hat{y}_{t}+\rho \mu_{y, t}, \\
\dot{\mu}_{\theta, t}=-\alpha_{\theta}\left(\hat{\theta}_{t}+\bar{\theta}-\alpha_{\psi} \lambda_{h, t}\right)-\Gamma+\rho \mu_{\theta, t} \\
(1-\alpha) \mu_{y, t}+\mu_{\theta, t}=0 .
\end{gathered}
$$

This yields

$$
\hat{y}_{t}+\frac{\alpha_{\theta}}{1-\alpha}\left(\hat{\theta}_{t}+\bar{\theta}-\alpha_{\psi} \lambda_{h, t}\right)=\frac{-1}{1-\alpha} \Gamma,
$$

which we can differentiate and combine with the Euler equation to get

$$
i_{t}=i_{t}^{*}+\frac{1}{1-\alpha+\frac{\alpha_{\theta}}{1-\alpha}}\left(\bar{r}_{t}-i_{t}^{*}\right)
$$


implying

$$
\hat{\theta}_{t}=\hat{\theta}_{0}+\int_{0}^{t} \frac{1}{1-\alpha+\frac{\alpha_{\theta}}{1-\alpha}}\left(\bar{r}_{s}-i_{s}^{*}+\frac{\alpha_{\theta} \alpha_{\psi}}{1-\alpha} \dot{\lambda}_{h, s}\right) d s .
$$

We therefore have

$$
\int_{0}^{\infty} \hat{\theta}_{t} e^{-\rho t} d t=\frac{1}{\rho} \hat{\theta}_{0}+\frac{1}{\rho} \int_{0}^{\infty} \frac{1}{1-\alpha+\frac{\alpha_{\theta}}{1-\alpha}}\left(\bar{r}_{t}-i_{t}^{*}+\frac{\alpha_{\theta} \alpha_{\psi}}{1-\alpha} \dot{\lambda}_{h, s}\right) e^{-\rho t} d t
$$

so that $\int \hat{\theta}_{t} e^{-\rho t} d t=0$ requires

$$
\hat{\theta}_{0}=-\int_{0}^{\infty} \frac{1}{1-\alpha+\frac{\alpha_{\theta}}{1-\alpha}}\left(\bar{r}_{t}-i_{t}^{*}+\frac{\alpha_{\theta} \alpha_{\psi}}{1-\alpha} \dot{\lambda}_{h, t}\right) e^{-\rho t} d t
$$

implying

$$
\hat{\theta}_{t}=-\int_{0}^{\infty} \frac{1}{1-\alpha+\frac{\alpha_{\theta}}{1-\alpha}}\left(\bar{r}_{t}-i_{t}^{*}+\frac{\alpha_{\theta} \alpha_{\psi}}{1-\alpha} \dot{\lambda}_{h, t}\right) e^{-\rho t} d t+\int_{0}^{t} \frac{1}{1-\alpha+\frac{\alpha_{\theta}}{1-\alpha}}\left(\bar{r}_{s}-i_{s}^{*}+\frac{\alpha_{\theta} \alpha_{\psi}}{1-\alpha} \dot{\lambda}_{h, s}\right) d s .
$$

The initial condition $\hat{y}_{0}-(1-\alpha) \hat{\theta}_{0}+\bar{s}_{0}=0$ and the optimality condition $\hat{y}_{0}+\frac{\alpha_{\theta}}{1-\alpha}\left(\hat{\theta}_{0}+\right.$ $\left.\bar{\theta}-\alpha_{\psi} \lambda_{h, 0}\right)=\frac{-1}{1-\alpha} \Gamma$ then imply that

$$
\frac{1}{1-\alpha} \Gamma=\int_{0}^{\infty}\left(\bar{r}_{t}-i_{t}^{*}+\frac{\alpha_{\theta} \alpha_{\psi}}{1-\alpha} \dot{\lambda}_{h, t}\right) e^{-\rho t} d t+\bar{s}_{0}-\frac{\alpha_{\theta}}{1-\alpha}\left(\bar{\theta}-\alpha_{\psi} \lambda_{h, 0}\right) .
$$

This implies that

$$
\begin{aligned}
\hat{y}_{t}=-\frac{1-\alpha}{1-\alpha+\frac{\alpha_{\theta}}{1-\alpha}} & \int_{0}^{\infty}\left(\bar{r}_{t}-i_{t}^{*}+\frac{\alpha_{\theta} \alpha_{\psi}}{1-\alpha} \dot{\lambda}_{h, t}\right) e^{-\rho t} d t \\
& -\frac{\frac{\alpha_{\theta}}{1-\alpha}}{1-\alpha+\frac{\alpha_{\theta}}{1-\alpha}} \int_{0}^{t}\left(\bar{r}_{s}-i_{s}^{*}+\frac{\alpha_{\theta} \alpha_{\psi}}{1-\alpha} \dot{\lambda}_{h, s}\right) d s-\bar{s}_{0}+\frac{\alpha_{\theta} \alpha_{\psi}}{1-\alpha}\left(\lambda_{h, t}-\lambda_{h, 0}\right) .
\end{aligned}
$$

\section{A.10 Proof of Proposition 9}

In the closed economy limit, the planning problem becomes

$$
\min \int e^{-\rho t}\left[\frac{1}{2} \alpha_{\pi} \pi_{H, t}^{2}+\frac{1}{2} \hat{y}_{t}^{2}\right] d t
$$

s.t.

$$
\dot{\pi}_{H, t}=\rho \pi_{H, t}-\hat{\kappa} \hat{y}_{t}
$$




$$
\begin{gathered}
\dot{\hat{y}}_{t}=i_{t}-i_{t}^{*}-\pi_{H, t}+i_{t}^{*}-\bar{r}_{t}, \\
\dot{\hat{\theta}}_{t}=i_{t}-i_{t}^{*} \\
\int \hat{\theta}_{t} e^{-\rho t} d t=0, \\
\hat{y}_{0}=\hat{\theta}_{0}-\bar{s}_{0} .
\end{gathered}
$$

Let $\Gamma$ be the multiplier on the budget constraint, and let $\mu_{x, t}$ and $\mu_{\theta, t}$ be the co-states. We find the following first-order conditions

$$
\begin{gathered}
\dot{\mu}_{y, t}=\rho \mu_{y, t}+\Gamma, \\
\dot{\hat{y}}_{t}=-\alpha_{\pi} \hat{\kappa} \pi_{H, t}+\hat{\kappa} \mu_{y, t}, \\
\dot{\pi}_{H, t}=\rho \pi_{H, t}-\hat{\kappa} \hat{y}_{t} .
\end{gathered}
$$

We can combine the second first order condition with the Euler equation to get

$$
\dot{\hat{\theta}}_{t}=\left(1-\alpha_{\pi} \hat{\kappa}\right) \pi_{H, t}+\hat{\kappa} \mu_{y, t}+\bar{r}_{t}-i_{t}^{*}
$$

Finally we have

$$
\hat{y}_{0}=-\Gamma
$$

We get

$$
\mu_{y, t}=e^{\rho t}\left[\mu_{y, 0}+\Gamma \frac{1}{\rho}\left(1-e^{-\rho t}\right)\right]=e^{\rho t}\left[\mu_{y, 0}+\Gamma \frac{1}{\rho}\right]-\Gamma \frac{1}{\rho},
$$

which requires

$$
\frac{1}{\rho} \Gamma=-\mu_{y, 0}
$$

and implies

$$
\mu_{y, t}=-\frac{1}{\rho} \Gamma .
$$

We are left with the following system:

$$
\begin{gathered}
\dot{\hat{y}}_{t}=-\alpha_{\pi} \hat{\kappa} \pi_{H, t}-\hat{\kappa} \frac{\Gamma}{\rho}, \\
\dot{\pi}_{H, t}=\rho \pi_{H, t}-\hat{\kappa} \hat{y}_{t}, \\
\hat{y}_{0}=-\Gamma,
\end{gathered}
$$


and then to solve for the value of $\Gamma$ such that the solution of the differential equation

$$
\dot{\hat{\theta}}_{t}=\left(1-\alpha_{\pi} \hat{\kappa}\right) \pi_{H, t}-\hat{\kappa} \frac{\Gamma}{\rho}+\bar{r}_{t}-i_{t}^{*}
$$

with the initial condition

$$
\hat{\theta}_{0}=\hat{y}_{0}+\bar{s}_{0}
$$

features

$$
\int e^{-\rho t} \hat{\theta}_{t} d t=0
$$

Let

$$
v=\frac{\rho-\sqrt{\rho^{2}+4 \alpha_{\pi}^{2} \hat{\kappa}^{2}}}{2}
$$

The closed-form solution is

$$
\begin{gathered}
\hat{y}_{t}=\Gamma \frac{1-\alpha_{\pi} \hat{\kappa}}{-v(\rho-v)}\left(e^{v t}-1\right)-\Gamma, \\
\pi_{H, t}=\Gamma \frac{1-\alpha_{\pi} \hat{\kappa}}{\alpha_{\pi}(\rho-v)}\left(e^{v t}-1\right)-\Gamma \frac{1}{\alpha_{\pi}}\left[\frac{-\rho\left(1-\alpha_{\pi} \hat{\kappa}\right)+\rho-v}{\rho(\rho-v)}\right], \\
i_{t}=\bar{r}_{t}+\left(1-\alpha_{\pi} \hat{\kappa}\right) \pi_{H, t}-\frac{\hat{\kappa}}{\rho} \Gamma=\bar{r}_{t}+\Gamma \frac{\left(1-\alpha_{\pi} \hat{\kappa}\right)^{2}}{\alpha_{\pi}(\rho-v)} e^{v t}-\frac{1}{\rho \alpha_{\pi}} \Gamma,
\end{gathered}
$$

where

$$
\Gamma=\left[\bar{s}_{0}+\int_{0}^{\infty} e^{-\rho t}\left(\bar{r}_{t}-i_{t}^{*}\right)\right]\left[1+\hat{\kappa}+\left(1-\alpha_{\pi} \hat{\kappa}\right)\left(\frac{1}{\rho^{2} \alpha_{\pi}}-\frac{1-\alpha_{\pi} \hat{\kappa}}{\alpha_{\pi}(\rho-v)^{2}}\right)\right] .
$$

\section{A.11 Proof of Proposition 10}

With flexible prices, we have $\hat{y}_{t}=-\frac{\alpha}{1+\phi} \hat{\theta}_{t}$ and we can drop the initial condition since the price of home goods can jump. Hence we are led to the planning problem

$$
\min _{\left\{\hat{\theta}_{t}\right\}} \frac{1}{2} \int_{0}^{\infty} e^{-\rho t}\left[\left(\frac{\alpha}{1+\phi}\right)^{2} \hat{\theta}_{t}^{2}+\alpha_{\theta}\left(\hat{\theta}_{t}+\alpha_{\psi} \bar{\theta}_{t}+\left(1-\alpha_{\psi}\right) \bar{\theta}_{0}\right)^{2}\right] d t
$$

subject to

$$
\int_{0}^{\infty} e^{-\rho t} \hat{\theta}_{t} d t=0
$$


Let $\Gamma$ be the multiplier on the budget constraint. The solution is given by

$$
\left[\left(\frac{\alpha}{1+\phi}\right)^{2}+\alpha_{\theta}\right] \hat{\theta}_{t}=-\alpha_{\theta} \alpha_{\psi} \bar{\theta}_{t}-\alpha_{\theta}\left(1-\alpha_{\psi}\right) \bar{\theta}_{0}-\Gamma .
$$

Since $\int_{0}^{\infty} e^{-\rho t} \bar{\theta}_{t} d t=0$ we find $\Gamma=-\alpha_{\theta}\left(1-\alpha_{\psi}\right) \bar{\theta}_{0}$ so that the solution is

$$
\begin{gathered}
\hat{\theta}_{t}=-\frac{\alpha_{\theta} \alpha_{\psi}}{\left(\frac{\alpha}{1+\phi}\right)^{2}+\alpha_{\theta}} \bar{\theta}_{t} \\
\hat{y}_{t}=\frac{\alpha}{1+\phi} \frac{\alpha_{\theta} \alpha_{\psi}}{\left(\frac{\alpha}{1+\phi}\right)^{2}+\alpha_{\theta}} \bar{\theta}_{t} .
\end{gathered}
$$

\section{A.12 Proof of Proposition 11}

We have following planning problem

$$
\min \int e^{-\rho t}\left[\frac{1}{2} \hat{y}_{t}^{2}+\alpha_{\theta} \frac{1}{2}\left(\hat{\theta}_{t}+\alpha_{\psi} \bar{\theta}_{t}+\left(1-\alpha_{\psi}\right) \bar{\theta}_{0}\right)^{2}\right]
$$

s.t.

$$
\begin{gathered}
\dot{\hat{y}}_{t}=(1-\alpha)\left(i_{t}-i_{t}^{*}-\dot{\bar{\theta}}_{t}\right)+i_{t}^{*}+\dot{\bar{\theta}}_{t}-\bar{r}_{t}, \\
\dot{\hat{\theta}}_{t}=i_{t}-i_{t}^{*}-\dot{\bar{\theta}}_{t}, \\
\int_{0}^{\infty} e^{-\rho t} \hat{\theta}_{t} d t=0, \\
\hat{y}_{0}=(1-\alpha) \hat{\theta}_{0}-\bar{s}_{0} .
\end{gathered}
$$

Let $\Gamma$ be the multiplier on the budget constraint and let $\mu_{x, t}$ and $\mu_{\theta, t}$ be the co-states. We find the following first-order conditions

$$
\begin{gathered}
\dot{\mu}_{y, t}=-\hat{y}_{t}+\rho \mu_{y, t}, \\
\dot{\mu}_{\theta, t}=-\alpha_{\theta}\left(\hat{\theta}_{t}+\alpha_{\psi} \bar{\theta}_{t}+\left(1-\alpha_{\psi}\right) \bar{\theta}_{0}\right)-\Gamma+\rho \mu_{\theta, t} \\
(1-\alpha) \mu_{y, t}+\mu_{\theta, t}=0 .
\end{gathered}
$$

This implies that

$$
\hat{y}_{t}+\frac{\alpha_{\theta}}{1-\alpha}\left(\hat{\theta}_{t}+\alpha_{\psi} \bar{\theta}_{t}+\left(1-\alpha_{\psi}\right) \bar{\theta}_{0}\right)=\frac{-1}{1-\alpha} \Gamma,
$$


and hence differentiating, we get

$$
-\frac{\alpha_{\theta}}{1-\alpha}\left(i_{t}-i_{t}^{*}-\dot{\bar{\theta}}_{t}\left(1-\alpha_{\psi}\right)\right)=(1-\alpha)\left(i_{t}-i_{t}^{*}-\dot{\bar{\theta}}_{t}\right)+i_{t}^{*}+\dot{\bar{\theta}}_{t}-\bar{r}_{t} .
$$

This can be rewritten as

$$
i_{t}=i_{t}^{*}+\frac{1}{1-\alpha+\frac{\alpha_{\theta}}{1-\alpha}}\left[\bar{r}_{t}+\left(\frac{\alpha_{\theta}\left(1-\alpha_{\psi}\right)}{1-\alpha}-\alpha\right) \dot{\bar{\theta}}_{t}-i_{t}^{*}\right],
$$

or

$$
i_{t}=i_{t}^{*}+\frac{\frac{\alpha}{1+\phi}\left[\frac{2-\alpha-(1-\alpha)^{2}}{(1-\alpha)^{2}}\right]}{1-\alpha+\frac{\alpha}{1+\phi} \frac{2-\alpha+(1-\alpha)^{2}}{(1-\alpha)^{2}}} \psi_{t}
$$

implying

$$
\tau_{t}=\frac{1}{1-\alpha+\frac{\alpha_{\theta}}{1-\alpha}}\left[\bar{r}_{t}-\psi_{t}-i_{t}^{*}-\frac{\alpha_{\theta} \alpha_{\psi}}{1-\alpha} \psi_{t}\right] .
$$

We also get

$$
\hat{\theta}_{t}+\bar{\theta}_{t}=\hat{\theta}_{0}+\bar{\theta}_{0}+\int_{0}^{t} \frac{1}{1-\alpha+\frac{\alpha_{\theta}}{1-\alpha}}\left(\bar{r}_{s}+\left(\frac{\alpha_{\theta}\left(1-\alpha_{\psi}\right)}{1-\alpha}-\alpha\right) \dot{\bar{\theta}}_{s}-i_{s}^{*}\right) d s
$$

or

$$
\hat{\theta}_{t}+\bar{\theta}_{t}=\hat{\theta}_{0}+\bar{\theta}_{0}+\int_{0}^{t} \frac{1}{1-\alpha+\frac{\alpha_{\theta}}{1-\alpha}}\left(\frac{\alpha_{\theta}\left(1-\alpha_{\psi}\right)}{1-\alpha}-\frac{\alpha}{1+\phi}\right) \psi_{s} d s
$$

which using $\int_{0}^{\infty} e^{-\rho t} \hat{\theta}_{t} d t=\int_{0}^{\infty} e^{-\rho t} \bar{\theta}_{t} d t=0$, we can rewrite as

$$
\hat{\theta}_{t}=-\bar{\theta}_{t}-\int_{0}^{\infty} \frac{\frac{\alpha}{1+\phi} \frac{2-\alpha-(1-\alpha)^{2}}{(1-\alpha)^{2}}}{1-\alpha+\frac{\alpha}{1+\phi} \frac{2-\alpha+(1-\alpha)^{2}}{(1-\alpha)^{2}}} \psi_{s} e^{-\rho s} d s+\int_{0}^{t} \frac{\frac{\alpha}{1+\phi} \frac{2-\alpha-(1-\alpha)^{2}}{(1-\alpha)^{2}}}{1-\alpha+\frac{\alpha}{1+\phi} \frac{2-\alpha+(1-\alpha)^{2}}{(1-\alpha)^{2}}} \psi_{s} d s
$$

i.e.

$$
\hat{\theta}_{t}=-\frac{1-\alpha}{1-\alpha+\frac{\alpha}{1+\phi} \frac{2-\alpha+(1-\alpha)^{2}}{(1-\alpha)^{2}}} \bar{\theta}_{t} .
$$

Finally, using the Euler equation and the initial condition, we find that

$$
\hat{y}_{t}=\left[(1-\alpha) \frac{1-\alpha+2 \frac{\alpha}{1+\phi}}{1-\alpha+\frac{\alpha}{1+\phi} \frac{2-\alpha+(1-\alpha)^{2}}{(1-\alpha)^{2}}}-\frac{1+\phi(1-\alpha)}{1+\phi}\right]\left[\int_{0}^{\infty} \psi_{t} e^{-\rho t} d t-\int_{0}^{t} \psi_{s} d s\right] .
$$




\section{A.13 Proof of Proposition 12}

In the closed economy limit, the planning problem becomes

$$
\min _{\left\{\pi_{H, t}, \hat{y}_{t}, i_{t}, \hat{\theta}_{t}\right\}} \frac{1}{2} \int_{0}^{\infty} e^{-\rho t}\left[\alpha_{\pi} \pi_{H, t}^{2}+\hat{y}_{t}^{2}\right] d t
$$

subject to

$$
\begin{gathered}
\dot{\pi}_{H, t}=\rho \pi_{H, t}-\hat{\kappa} \hat{y}_{t}, \\
\dot{y}_{t}=i_{t}-\pi_{H, t}-\rho, \\
\dot{\hat{\theta}}_{t}=i_{t}-\rho-\psi_{t}, \\
\int_{0}^{\infty} e^{-\rho t} \hat{\theta}_{t} d t=0, \\
\hat{y}_{0}=\hat{\theta}_{0}+\int_{0}^{\infty} \psi_{t} e^{-\rho t} d t .
\end{gathered}
$$

We guess and verify that $\pi_{H, t}=\hat{y}_{t}=0, \hat{\theta}_{t}=\int_{0}^{\infty} \psi_{s} e^{-\rho s} d s-\int_{0}^{t} \psi_{s} d s$, and $i_{t}=\rho$ is a solution.

\section{A.14 Proof of Proposition 13}

With capital controls we have

$$
\min _{\left\{\pi_{H, t}, \hat{y}_{t}, i_{t}, \hat{\theta}_{t}, e_{t}\right\}} \frac{1}{2} \int_{0}^{\infty} e^{-\rho t}\left[\alpha_{\pi} \pi_{H, t}^{2}+\hat{y}_{t}^{2}+\alpha_{\theta}\left(\hat{\theta}_{t}+\alpha_{\psi} \bar{\theta}_{t}+\left(1-\alpha_{\psi}\right) \bar{\theta}_{0}\right)^{2}\right] d t
$$

subject to

$$
\begin{gathered}
\dot{\pi}_{H, t}=\rho \pi_{H, t}-\hat{\kappa} \hat{y}_{t}-\lambda \alpha \hat{\theta}_{t}, \\
\dot{\hat{y}}_{t}=(1-\alpha)\left(i_{t}-i_{t}^{*}-\dot{e}_{t}-\psi_{t}\right)-\pi_{H, t}+i_{t}^{*}+\dot{e}_{t}+\psi_{t}-\bar{r}_{t}, \\
\dot{\hat{\theta}}_{t}=i_{t}-i_{t}^{*}-\dot{e}_{t}-\psi_{t}, \\
\int_{0}^{\infty} e^{-\rho t} \hat{\theta}_{t} d t=0, \\
\hat{y}_{0}=(1-\alpha) \hat{\theta}_{0}+e_{0}-\bar{s}_{0} .
\end{gathered}
$$


Note that this allows the planner to control $\hat{\theta}_{t}$ and $\hat{y}_{t}$ independently. We can drop the initial condition. We can therefore rewrite the problem as

$$
\min _{\left\{\pi_{H, t}, \hat{y}_{t}, \hat{\theta}_{t}\right\}} \frac{1}{2} \int_{0}^{\infty} e^{-\rho t}\left[\alpha_{\pi} \pi_{H, t}^{2}+\hat{y}_{t}^{2}+\alpha_{\theta}\left(\hat{\theta}_{t}+\alpha_{\psi} \bar{\theta}_{t}+\left(1-\alpha_{\psi}\right) \bar{\theta}_{0}\right)^{2}\right] d t
$$

subject to

$$
\begin{gathered}
\dot{\pi}_{H, t}=\rho \pi_{H, t}-\hat{\kappa} \hat{y}_{t}-\lambda \alpha \hat{\theta}_{t}, \\
\int_{0}^{\infty} e^{-\rho t} \hat{\theta}_{t} d t=0 .
\end{gathered}
$$

The FOCs are

$$
\begin{gathered}
-\dot{\mu}_{\pi, t}=\alpha_{\pi} \pi_{H, t}, \\
\hat{y}_{t}-\hat{\kappa} \mu_{\pi, t}=0, \\
\alpha_{\theta}\left(\hat{\theta}_{t}+\alpha_{\psi} \bar{\theta}_{t}+\left(1-\alpha_{\psi}\right) \bar{\theta}_{0}\right)+\Gamma-\lambda \alpha \mu_{\pi, t}=0 .
\end{gathered}
$$

Note that this implies the following formula for capital controls

$$
\tau_{t}=-\alpha_{\psi} \psi_{t}+\frac{\lambda \alpha}{\alpha_{\theta}} \dot{\mu}_{\pi, t}=-\alpha_{\psi} \psi_{t}-\frac{\lambda \alpha}{\alpha_{\theta}} \alpha_{\pi} \pi_{H, t}
$$

This formula depends on the endogenous object $\pi_{H, t}$ which we determine in closed form below.

We have the following system of differential equations

$$
\begin{gathered}
\dot{\pi}_{H, t}=\rho \pi_{H, t}-\left(\hat{\kappa}^{2}+\frac{(\lambda \alpha)^{2}}{\alpha_{\theta}}\right) \mu_{\pi, t}+\lambda \alpha\left[\frac{\Gamma}{\alpha_{\theta}}+\alpha_{\psi} \bar{\theta}_{t}+\left(1-\alpha_{\psi}\right) \bar{\theta}_{0}\right], \\
\dot{\mu}_{\pi, t}=-\alpha_{\pi} \pi_{H, t},
\end{gathered}
$$

with $\mu_{\pi, 0}=0$.

$$
\text { Let } X_{t}=\left[\pi_{H, t}, \mu_{\pi, t}\right]^{\prime}, A=\left[\begin{array}{cc}
\rho & -\left(\hat{\kappa}^{2}+\frac{(\lambda \alpha)^{2}}{\alpha_{\theta}}\right) \\
-\alpha_{\pi} & 0
\end{array}\right] \text {, and } B_{t}=\left[\lambda \alpha\left[\frac{\Gamma}{\alpha_{\theta}}+\alpha_{\psi} \bar{\theta}_{t}+\left(1-\alpha_{\psi}\right) \bar{\theta}_{0}\right], 0\right]^{\prime} \text {. }
$$

We can rewrite this as

$$
\dot{X}_{t}=A X_{t}+B_{t}
$$

The solution is

$$
X_{t}=e^{A t}\left[X_{0}+\int_{0}^{t} e^{-A s} B_{s} d s\right]
$$


The matrix $A$ has exactly one negative eigenvalue

$$
v=\frac{\rho-\sqrt{\rho^{2}+4 \alpha_{\pi}\left(\hat{\kappa}^{2}+\frac{(\lambda \alpha)^{2}}{\alpha_{\theta}}\right)}}{2} .
$$

Let $V_{v}=\left[v_{v}, 1\right]^{\prime}$ be the corresponding eigenvector where $v_{v}=-\frac{1}{\alpha_{\pi}}$. We need $X_{0}+$ $\int_{0}^{\infty} e^{-A s} B_{s} d s$ to be co-linear with $V_{v}$, i.e. we need

$$
\left[\pi_{H, 0}, 0\right]^{\prime}+\int_{0}^{\infty} e^{-A s} B_{s} d s=\mu\left[v_{v}, 1\right]^{\prime}
$$

We can project this equation using $E_{1}=[1,0]^{\prime}$ and $E_{2}=[0,1]^{\prime}$. We find

$$
\begin{gathered}
\int_{0}^{\infty} E_{2}^{\prime} e^{-A s} B_{s} d s=\mu, \\
\pi_{H, 0}=-\int_{0}^{\infty} E_{1}^{\prime} e^{-A s} B_{s} d s-\frac{1}{\alpha_{\pi}} \int_{0}^{\infty} E_{2}^{\prime} e^{-A s} B_{s} d s,
\end{gathered}
$$

and $X_{0}=\left[\pi_{H, 0}, 0\right]^{\prime}$. Hence we have

$$
X_{t}=e^{A t}\left[X_{0}+\int_{0}^{\infty} e^{-A s} B_{s} d s\right]-\int_{t}^{\infty} e^{-A(s-t)} B_{s} d s,
$$

and finally

$$
X_{t}=e^{v t}\left(\int_{0}^{\infty} E_{2}^{\prime} e^{-A s} B_{s} d s\right)\left[-\frac{1}{\alpha_{\pi}}, 1\right]^{\prime}-\int_{t}^{\infty} e^{-A(s-t)} B_{s} d s .
$$

We can now try to determine $\Gamma$. For this, we rewrite $B_{t}=\frac{\lambda}{\alpha_{\theta}} \Gamma E_{1}+\tilde{B}_{t}$ where $\tilde{B}_{t}=$ $\left[\lambda \alpha\left[\alpha_{\psi} \bar{\theta}_{t}+\left(1-\alpha_{\psi}\right) \bar{\theta}_{0}\right], 0\right]^{\prime}$. We then write

$$
X_{t}=e^{v t}\left(\frac{\lambda}{\alpha_{\theta}} \Gamma E_{2}^{\prime} A^{-1} E_{1}+\int_{0}^{\infty} E_{2}^{\prime} e^{-A s} \tilde{B}_{s} d s\right)\left[-\frac{1}{\alpha_{\pi}}, 1\right]^{\prime}-\int_{t}^{\infty} e^{-A(s-t)} \tilde{B}_{s} d s-\Gamma \frac{\lambda}{\alpha_{\theta}} A^{-1} E_{1},
$$

and we can use the budget constraint to find $\Gamma$ as follows. First use

$$
\alpha_{\theta}\left(\hat{\theta}_{t}+\alpha_{\psi} \bar{\theta}_{t}+\left(1-\alpha_{\psi}\right) \bar{\theta}_{0}\right)+\Gamma=\lambda \alpha E_{2}^{\prime} X_{t}
$$

and integrate to find

$$
\alpha_{\theta}\left(\left(1-\alpha_{\psi}\right) \bar{\theta}_{0}\right)+\Gamma=\lambda \alpha \rho \int_{0}^{\infty} e^{-\rho t} E_{2}^{\prime} X_{t} d t
$$


which leads to

$$
\begin{aligned}
& \alpha_{\theta}\left(\left(1-\alpha_{\psi}\right) \bar{\theta}_{0}\right)+\Gamma= \\
& \lambda \alpha \rho\left[\frac{1}{\rho+v}\left(\frac{\lambda}{\alpha_{\theta}} \Gamma E_{2}^{\prime} A^{-1} E_{1}+\int_{0}^{\infty} E_{2}^{\prime} e^{-A s} B_{s} d s\right)-\int_{0}^{\infty} e^{-\rho t} \int_{t}^{\infty} E_{2}^{\prime} e^{-A(s-t)} \tilde{B}_{s} d s d t-\Gamma \frac{1}{\rho} \frac{\lambda}{\alpha_{\theta}} E_{2}^{\prime} A^{-1} E_{1}\right],
\end{aligned}
$$

or

$$
\Gamma=\frac{-\alpha_{\theta}\left(1-\alpha_{\psi}\right) \bar{\theta}_{0}+\lambda \alpha \rho\left[\frac{1}{\rho+v}\left(\int_{0}^{\infty} E_{2}^{\prime} e^{-A s} B_{s} d s\right)-\int_{0}^{\infty} e^{-\rho t} \int_{t}^{\infty} E_{2}^{\prime} e^{-A(s-t)} \tilde{B}_{s} d s d t\right]}{1+\frac{v}{\rho+v} \frac{\lambda^{2} \alpha}{\alpha_{\theta}} E_{2}^{\prime} A^{-1} E_{1}} .
$$

Imagine now that prices are rigid. Then the problem simplifies to

$$
\min _{\left\{\pi_{H, t}, \hat{y}_{t}, i_{t}, \hat{\theta}_{t}, e_{t}\right\}} \frac{1}{2} \int_{0}^{\infty} e^{-\rho t}\left[\hat{y}_{t}^{2}+\alpha_{\theta}\left(\hat{\theta}_{t}+\alpha_{\psi} \bar{\theta}_{t}+\left(1-\alpha_{\psi}\right) \bar{\theta}_{0}\right)^{2}\right] d t
$$

subject to

$$
\int_{0}^{\infty} e^{-\rho t} \hat{\theta}_{t} d t=0 .
$$

The solution is $\hat{y}_{t}=0$ and $\hat{\theta}_{t}=-\alpha_{\psi} \bar{\theta}_{t}$, which implies

$$
\tau_{t}=-\alpha_{\psi} \psi_{t}
$$

We can also compute $e_{t}$. For that we use

$$
\begin{gathered}
\dot{\hat{y}}_{t}=(1-\alpha)\left(i_{t}-\rho\right)+\alpha\left(\dot{e}_{t}+\psi_{t}\right)-\frac{\alpha \phi}{1+\phi} \psi_{t}, \\
\dot{\hat{\theta}}_{t}=\left(i_{t}-\rho\right)-\left(\dot{e}_{t}+\psi_{t}\right), \\
\hat{y}_{0}=(1-\alpha) \hat{\theta}_{0}+e_{0}-\bar{s}_{0} .
\end{gathered}
$$

This yields

$$
\begin{gathered}
\dot{e}_{t}=-\left[\frac{\alpha}{1+\phi}+(1-\alpha)\left(1-\alpha_{\psi}\right)\right] \psi_{t} \\
e_{0}=\left[\frac{1+\phi(1-\alpha)}{1+\phi}-(1-\alpha) \alpha_{\psi}\right] \int_{0}^{\infty} \psi_{t} e^{-\rho t} d t .
\end{gathered}
$$

Hence at least for small $\alpha$, in response to a negative risk premium shock that mean reverts to zero, the exchange rate initially appreciates and then depreciates over time. 
This solution is clearly different from the one with fixed exchange rate which entails

$$
\tau_{t}=-\frac{1-\alpha+\frac{2 \alpha}{1+\phi}}{1-\alpha+\frac{\alpha}{1+\phi} \frac{2-\alpha+(1-\alpha)^{2}}{(1-\alpha)^{2}}} \psi_{t}
$$

How can we compare them? In both cases, capital controls are used to lean agains the wind. But the former leans less agains the wind than the latter.

We can also compare the solution to the flexible price solution

$$
\tau_{t}=-\frac{\alpha_{\theta} \alpha_{\psi}}{\left(\frac{\alpha}{1+\phi}\right)^{2}+\alpha_{\theta}} \psi_{t}
$$

so that we see that capital controls are always used more with rigid prices and flexible exchange rate than with flexible prices. However, this difference disappears when $\alpha \rightarrow 0$. There the exchange rate required to implement this allocation with sticky prices is

$$
e_{t}=-\frac{1+\phi(1-\alpha)}{1+\phi} \frac{\frac{\alpha}{(1+\phi)^{2}}+\alpha_{\theta}\left(1-\alpha_{\psi}\right)}{\frac{\alpha}{(1+\phi)^{2}}+\alpha_{\theta}}\left[\int_{0}^{t} \psi_{s} d s-\int_{0}^{\infty} e^{-\rho t} \psi_{t} d t\right],
$$

and in particular

$$
e_{0}=\frac{1+\phi(1-\alpha)}{1+\phi} \frac{\frac{\alpha}{(1+\phi)^{2}}+\alpha_{\theta}\left(1-\alpha_{\psi}\right)}{\frac{\alpha}{(1+\phi)^{2}}+\alpha_{\theta}} \int_{0}^{\infty} e^{-\rho t} \psi_{t} d t
$$

\section{A.15 Planning Problem with Sticky Wages}

Here we allow any path for $e_{t}$. To consider the case with a fixed exchange rate we can impose the further condition that $e_{t}=0$. The planning problem is

$$
\begin{gathered}
\min _{\left\{\pi_{H, t}, \pi_{t}^{w}, \hat{y}_{t}, i_{t}, \hat{\theta}_{t}, \hat{\omega}_{t}, e_{t}\right\}} \frac{1}{2} \int_{0}^{\infty} e^{-\rho t}\left[\alpha_{\pi} \pi_{H, t}^{2}+\alpha_{\pi^{w}}\left(\pi_{t}^{w}\right)^{2}+\hat{y}_{t}^{2}+\alpha_{\theta} \hat{\theta}_{t}^{2}\right] d t \\
\dot{\pi}_{t}^{w}=\rho \pi_{t}^{w}-\lambda_{w}[(1-\alpha)+\phi] \hat{y}_{t}-\lambda_{w} \alpha(2-\alpha) \hat{\theta}_{t}+\lambda_{w} \hat{\omega}_{t} \\
\dot{\pi}_{H, t}=\rho \pi_{H, t}-\lambda \hat{\omega}_{t}-\lambda \alpha \hat{y}_{t}+\lambda \alpha(1-\alpha) \hat{\theta}_{t} \\
\dot{\hat{y}}_{t}=(1-\alpha) i_{t}-\left(\pi_{H, t}+\bar{r}_{t}\right)+\alpha\left(i_{t}^{*}+\dot{e}_{t}\right) \\
\dot{\hat{\theta}}_{t}=i_{t}-i_{t}^{*}-\dot{e}_{t} \\
\dot{\hat{\omega}}_{t}=\pi_{t}^{w}-(1-\alpha) \pi_{H, t}-\alpha\left(\pi_{t}^{*}+\dot{e}_{t}\right)-\dot{\bar{\omega}}_{t}
\end{gathered}
$$


together with the initial conditions

$$
\begin{gathered}
\hat{\omega}_{0}=-\bar{\omega}_{0}-\alpha e_{0}, \\
e_{0}-\bar{s}_{0}=\hat{y}_{0}-(1-\alpha) \hat{\theta}_{0},
\end{gathered}
$$

and the country budget constraint

$$
\int_{0}^{\infty} e^{-\rho t} \hat{\theta}_{t}=0
$$

By putting a multiplier $\Gamma$ on the country budget constraint and incorporating it in the objective, we are left with an optimal control problem with state variables $\pi_{H, t}, \pi_{t}^{w}, \hat{y}_{t}, \hat{\theta}_{t}$, $\hat{\omega}_{t}$, and control variables $i_{t}$ and $\dot{e}_{t}$.

\section{A.16 Proof of Proposition 14}

We first look at the case of rigid prices and wages. The planning problem simplifies to

$$
\min _{\left\{\pi_{H, t}, \pi_{t}^{w}, \hat{y}_{t}, i_{t}, \hat{\theta}_{t}, e_{t}\right\}} \frac{1}{2} \int_{0}^{\infty} e^{-\rho t}\left[\hat{y}_{t}^{2}+\alpha_{\theta} \hat{\theta}_{t}^{2}\right] d t
$$

subject to

$$
\begin{gathered}
\dot{\hat{y}}_{t}=(1-\alpha) i_{t}-\bar{r}_{t}+\alpha\left(i_{t}^{*}+\dot{e}_{t}\right), \\
\dot{\hat{\theta}}_{t}=i_{t}-i_{t}^{*}-\dot{e}_{t}, \\
\int_{0}^{\infty} e^{-\rho t} \hat{\theta}_{t}=0 .
\end{gathered}
$$

Clearly the optimal solution is to set $\hat{\theta}_{t}=0, i_{t}=\bar{r}_{t}$ and set $\dot{e}_{t}$ equal to $i_{t}-i_{t}^{*}$, so that optimal capital controls are zero.

In the closed economy limit $\alpha \rightarrow 0$, capital controls are useless since the loss function can be solved as the solution of the following planning problem

$$
\min _{\left\{\pi_{H, t}, \pi_{t}^{w}, \hat{y}_{t}, i_{t}, \hat{\omega}_{t}\right\}} \frac{1}{2} \int_{0}^{\infty} e^{-\rho t}\left[\alpha_{\pi} \pi_{H, t}^{2}+\alpha_{\pi^{w}}\left(\pi_{t}^{w}\right)^{2}+\hat{y}_{t}^{2}\right] d t
$$

subject to

$$
\begin{gathered}
\dot{\pi}_{t}^{w}=\rho \pi_{t}^{w}-\lambda_{w}(1+\phi) \hat{y}_{t}+\lambda_{w} \hat{\omega}_{t} \\
\dot{\pi}_{H, t}=\rho \pi_{H, t}-\lambda \hat{\omega}_{t},
\end{gathered}
$$




$$
\begin{aligned}
& \dot{\hat{y}}_{t}=i_{t}-\left(\pi_{H, t}+\bar{r}_{t}\right), \\
& \dot{\hat{\omega}}_{t}=\pi_{t}^{w}-\pi_{H, t}-\dot{\bar{\omega}}_{t} .
\end{aligned}
$$

and the initial condition

$$
\hat{\omega}_{0}=-\bar{\omega}_{0}
$$

Note also that this system is identical to that of a closed economy with sticky prices and sticky wages.

\section{A.17 Proof of Propositions 16 and 17}

We provide the proof for Proposition 16. The proof of Proposition 17 is almost identical.

We start by showing that at the coordinated solution, the disaggregated variables $\tilde{\pi}_{H, t^{\prime}}^{i}$ $\tilde{y}_{t}^{i}, \tilde{\theta}_{t}^{i}$ that solve the disaggregated planning problem (30) are equivalent to the solution to the component planning problem (31). Note that the family of problems in (31) constitute a relaxed version of the problem in (30), since it is identical except that the two aggregation constraints (26) and (27) are dropped. Also, the component planning problems (31) for any two different countries $i \in[0,1]$ are identical linear quadratic problems, except for possibly different paths for the forcing variable $\overline{\bar{s}}_{t}^{i}$. We note three facts. First, if $\overline{\bar{s}}_{t}^{i}=0$ for all $t \geq 0$ then the solution to (31) entails $\tilde{\pi}_{H, t}^{i}=\tilde{y}_{t}^{i}=\tilde{\theta}_{t}^{i}=0$. Second, the solution to (31) must be linear in the path of the forcing variable $\left\{\overline{\bar{s}}_{t}^{i}\right\}_{t \geq 0}$. Third, we have by definition $\int_{0}^{1} \bar{s}_{t}^{i} d i=0$. Putting these three facts together implies that the family of solutions to (31) automatically satisfy the two aggregation constraints $0=\int_{0}^{1} \tilde{y}_{t}^{i} d i$ and $0=\int_{0}^{1} \tilde{\pi}_{H, t}^{i} d i$ (equations (26), and (27)) and therefore coincide with the solution to (30).

Next we show that that at the uncoordinated solution, the disaggregated variables $\tilde{\pi}_{H, t}^{i}, \tilde{y}_{t}^{i}, \tilde{\theta}_{t}^{i}$ also solve the component planning problems (31). First note that the uncoordinated component planning problem (32) coincides with (31) when $\hat{y}_{t}^{*}=\pi_{t}^{*}=0$ for all $t \geq 0$. Second, for any path of $\left\{\hat{\hat{y}}_{t}^{*}, \pi_{t}^{*}, \overline{\bar{s}}_{t}^{i}\right\}_{t \geq 0}$ the problem is linear quadratic and the solution is linear in these variables. Third, an uncoordinated solution $\left\{\hat{y}_{t}^{*}, \pi_{t}^{*}\right\}_{t \geq 0} \in \mathcal{F}$ requires that the associated solutions to (32) satisfy $0=\int_{0}^{1} \tilde{y}_{t}^{i} d i$ and $0=\int_{0}^{1} \tilde{\pi}_{H, t}^{i} d i$. Putting these three facts together implies that the family of solutions to (32) for $\left\{\hat{\hat{y}}_{t}^{*}, \pi_{t}^{*}\right\}_{t \geq 0} \in \mathcal{F}$ coincides with the solution of the component planning problem (31).

The derivation of the feasible set can be found in the next subsection. 


\section{A.18 Derivation of the feasible sets $\mathcal{F}$ and $\mathcal{F}^{\prime}$ in Propositions 16 and 17}

We deal with the case where the labor tax is set at its uncoordinated level. The other case is similar.

The uncoordinated component planning problem (32) is linear quadratic, so its solution, for given $\left\{\hat{y}_{t}^{*}, \pi_{t}^{*}\right\}_{t \geq 0}$, is linear in $\left\{\bar{s}_{t}^{i}\right\}_{t \geq 0}$. Then, since $\int_{0}^{1} \bar{s}_{t}^{i} d i=0$, it follows that the solution to (32) with $\left\{\hat{y}_{t}^{*}, \pi_{t}^{*}\right\}_{t \geq 0}$ satisfies the aggregation constraints $0=\int_{0}^{1} \tilde{y}_{t}^{i} d i$ and $0=\int_{0}^{1} \tilde{\pi}_{H, t}^{i} d i$ (equations (26), and (27)) if and only if the solution with $\bar{s}_{t}^{i}=0$ for all $t \geq 0$ yields $\tilde{\pi}_{H, t}^{i}=\tilde{y}_{t}^{i}=\tilde{\theta}_{t}^{i}=0$ for all $t \geq 0$.

Thus, we have established that $\left\{\hat{y}_{t}^{*}, \pi_{t}^{*}\right\}_{t \geq 0} \in \mathcal{F}$ if and only if $\dot{\pi}_{t}^{*}=\rho \pi_{t}^{*}-\hat{\kappa}_{\hat{y}}^{*}$ and and the following problem yields $\tilde{\pi}_{H, t}=\tilde{y}_{t}=\tilde{\theta}_{t}=0$ :

$$
\min \frac{1}{2} \int_{0}^{\infty} e^{-\rho t}\left[\alpha_{\pi}\left(\tilde{\pi}_{H, t}\right)^{2}+2 \alpha_{\pi} \pi_{t}^{*} \tilde{\pi}_{H, t}+\left(\tilde{y}_{t}\right)^{2}+2 \hat{y}_{t}^{*} \tilde{y}_{t}+\alpha_{\theta}\left(\tilde{\theta}_{t}\right)^{2}\right] d t
$$

subject to

$$
\begin{gathered}
\dot{\tilde{\pi}}_{H, t}=\rho \tilde{\pi}_{H, t}-\hat{\kappa} \tilde{y}_{t}-\lambda \alpha \tilde{\theta}_{t}, \\
\dot{\tilde{y}}_{t}=(1-\alpha) \dot{\tilde{\theta}}_{t}-\tilde{\pi}_{H, t}, \\
\int_{0}^{\infty} e^{-\rho t} \tilde{\theta}_{t} d t=0, \\
\tilde{y}_{0}=(1-\alpha) \tilde{\theta}_{0},
\end{gathered}
$$

where the minimization is over the variables $\tilde{\pi}_{H, t}, \tilde{y}_{t}, \tilde{\theta}_{t}$, taking $\hat{y}_{t}^{*}$, and $\pi_{t}^{*}$ as given. We put a multiplier $\Gamma$ on the constraint $\int e^{-\rho t} \tilde{\theta}_{t} d t=0$ and incorporate it in the objective to obtain an optimal control problem. The first-order conditions are

$$
\begin{gathered}
-\dot{\mu}_{\pi, t}=\alpha_{\pi} \tilde{\pi}_{H, t}+\alpha_{\pi} \pi_{t}^{*}-\mu_{y, t}, \\
\rho \mu_{y, t}-\dot{\mu}_{y, t}=\tilde{y}_{t}+\hat{y}_{t}^{*}-\hat{\kappa} \mu_{\pi, t}, \\
\rho \mu_{\theta, t}-\dot{\mu}_{\theta, t}=\alpha_{\theta} \tilde{\theta}_{t}+\Gamma-\lambda \alpha \mu_{\pi, t}, \\
(1-\alpha) \mu_{y, t}+\mu_{\theta, t}=0 \\
\mu_{\pi, 0}=0 .
\end{gathered}
$$

We can combine these equations to get

$$
\mu_{\pi, t}=\frac{1-\alpha}{\hat{\kappa}(1-\alpha)+\lambda \alpha} \tilde{y}_{t}+\frac{\alpha \theta}{\hat{\kappa}(1-\alpha)+\lambda \alpha} \tilde{\theta}_{t}+\frac{\Gamma}{\hat{\kappa}(1-\alpha)+\lambda \alpha}+\frac{1-\alpha}{\hat{\kappa}(1-\alpha)+\lambda \alpha} \hat{y}_{t}^{*},
$$


leading to the following reduced system (which substitutes both $\mu_{\pi, t}$ and $\mu_{\theta, t}$ out):

$$
\begin{gathered}
\frac{1-\alpha}{\hat{\kappa}(1-\alpha)+\lambda \alpha} \dot{\tilde{y}}_{t}+\frac{\alpha_{\theta}}{\hat{\kappa}(1-\alpha)+\lambda \alpha} \dot{\tilde{\theta}}_{t}+\frac{1-\alpha}{\hat{\kappa}(1-\alpha)+\lambda \alpha} \dot{\hat{y}}_{t}^{*}=-\alpha_{\pi} \tilde{\pi}_{H, t}+\mu_{y, t}-\alpha_{\pi} \pi_{t}^{*}, \\
\rho \mu_{y, t}-\dot{\mu}_{y, t}=\frac{\lambda \alpha}{\hat{\kappa}(1-\alpha)+\lambda \alpha} \tilde{y}_{t}-\frac{\hat{\kappa} \alpha_{\theta}}{\hat{\kappa}(1-\alpha)+\lambda \alpha} \tilde{\theta}_{t}-\frac{\hat{\kappa} \Gamma}{\hat{\kappa}(1-\alpha)+\lambda \alpha}+\frac{\lambda \alpha}{\hat{\kappa}(1-\alpha)+\lambda \alpha} \hat{y}_{t}^{*}, \\
\dot{\tilde{\pi}}_{H, t}=\rho \tilde{\pi}_{H, t}-\hat{\kappa} \tilde{y}_{t}-\lambda \alpha \tilde{\theta}_{t}, \\
\dot{\tilde{y}}_{t}=(1-\alpha) \dot{\tilde{\theta}}_{t}-\tilde{\pi}_{H, t}, \\
\tilde{y}_{0}=(1-\alpha) \tilde{\theta}_{0}, \\
\frac{1-\alpha}{\hat{\kappa}(1-\alpha)+\lambda \alpha} \tilde{y}_{0}+\frac{\alpha_{\theta}}{\hat{\kappa}(1-\alpha)+\lambda \alpha} \tilde{\theta}_{0}+\frac{\Gamma}{\hat{\kappa}(1-\alpha)+\lambda \alpha}+\frac{1-\alpha}{\hat{\kappa}(1-\alpha)+\lambda \alpha} \hat{y}_{0}^{*}=0 .
\end{gathered}
$$

In order for $\tilde{\pi}_{H, t}=\tilde{y}_{t}=\tilde{\theta}_{t}=0$ to be the solution, we must then have

$$
\begin{gathered}
\frac{1-\alpha}{\hat{\kappa}(1-\alpha)+\lambda \alpha} \dot{\hat{y}}_{t}^{*}=\mu_{y, t}-\alpha_{\pi} \pi_{t}^{*}, \\
\rho \mu_{y, t}-\dot{\mu}_{y, t}=-\frac{\hat{\kappa} \Gamma}{\hat{\kappa}(1-\alpha)+\lambda \alpha}+\frac{\lambda \alpha}{\hat{\kappa}(1-\alpha)+\lambda \alpha} \hat{y}_{t}^{*}, \\
\frac{\Gamma}{\hat{\kappa}(1-\alpha)+\lambda \alpha}+\frac{1-\alpha}{\hat{\kappa}(1-\alpha)+\lambda \alpha} \hat{y}_{0}^{*}=0,
\end{gathered}
$$

We seek bounded solutions. Combining these three equations and using that $\pi_{t}^{*}=$ $\kappa \int e^{-\rho(s-t)} \hat{y}_{s}^{*} d s$ we obtain

$$
\dot{\hat{y}}_{t}^{*}=-\frac{1}{\rho} \hat{\kappa} \hat{y}_{0}^{*}-\frac{1}{(1-\alpha) \hat{\kappa}}\left(\lambda \alpha+(\hat{\kappa}(1-\alpha)+\lambda \alpha) \alpha_{\pi} \hat{\kappa}\right) \pi_{t}^{*},
$$

which together with $\pi_{t}^{*}=\kappa \int e^{-\rho(s-t)} \hat{\hat{y}}_{s}^{*} d s$ fully characterizes the feasibility set $\mathcal{F}$.

To determine the dimensionality of $\mathcal{F}$ we then must solve the following system of differential equations

$$
\begin{gathered}
\dot{\hat{y}}_{t}^{*}=-\frac{1}{\rho} \hat{\kappa} \hat{y}_{0}^{*}-\frac{\lambda \alpha+(\hat{\kappa}(1-\alpha)+\lambda \alpha) \alpha_{\pi} \hat{\kappa}}{(1-\alpha) \hat{\kappa}} \pi_{t}^{*}, \\
\dot{\pi}_{t}^{*}=\rho \pi_{t}^{*}-\hat{\kappa} \hat{y}_{t}^{*} .
\end{gathered}
$$


Let $X_{t}=\left[\hat{y}_{t}^{*}, \pi_{t}^{*}\right]^{\prime}, B=\left[-\frac{1}{\rho} \hat{\kappa} \hat{y}_{0}^{*}, 0\right]^{\prime}$ and

$$
A=\left[\begin{array}{cc}
0 & -\frac{1}{(1-\alpha) \hat{\kappa}}\left(\lambda \alpha+(\hat{\kappa}(1-\alpha)+\lambda \alpha) \alpha_{\pi} \hat{\kappa}\right) \\
-\hat{\kappa} & \rho
\end{array}\right] .
$$

We have $\dot{X}_{t}=A X_{t}+B$.

We need to find the eigenvalues of $A$. The characteristic polynomial of $A$ is given by $x^{2}-\rho x-\frac{\lambda \alpha+(\hat{\kappa}(1-\alpha)+\lambda \alpha) \alpha_{\pi} \hat{\kappa}}{1-\alpha}$. This implies that $A$ two real eigenvalues of opposite signs. Let $v$ be the negative eigenvalue. Denote the corresponding eigenvector by $V_{v}$.

Let $E_{1}=[1,0]^{\prime}$ and $E_{2}=[0,1]^{\prime}$. We must then solve the system of two equations in three unknowns $\left(X_{0}=\left[\hat{y}_{0}^{*}, \pi_{0}^{*}\right]^{\prime}\right.$ and $\left.\alpha_{v}\right)$ :

$$
E_{i}^{\prime} X_{0}-\frac{1}{\rho} \hat{\kappa}\left(E_{1}^{\prime} X_{0}\right)\left(E_{i}^{\prime} A^{-1} E_{1}\right)=\alpha_{v} E_{i}^{\prime} V_{v}
$$

And we have

$$
X_{t}=\alpha_{v} e^{v t} V_{v}+\frac{1}{\rho} \hat{\kappa}\left(E_{1}^{\prime} X_{0}\right) A^{-1} E_{1}
$$

so that $\lim _{t \rightarrow \infty} X_{t}=\frac{1}{\rho} \hat{\kappa}\left(E_{1}^{\prime} X_{0}\right) A^{-1} E_{1}$. This means that the feasible set is a one-dimensional linear space. Moreover, the set of limits is also one-dimensional. This implies that the set of possible long-term limits for $\hat{y}_{t}^{*}$ and $\pi_{t}^{*}$ is the locus $0=\rho \pi_{t}^{*}-\hat{\kappa} \hat{y}_{t}^{*}$.

\section{A.19 The Ramsey Problem}

We treat the uncoordinated labor tax case. The coordinated labor tax case is similar. We want to solve

$$
\min \frac{1}{2} \int_{0}^{\infty} e^{-\rho t}\left[\alpha_{\pi}\left(\pi_{t}^{*}\right)^{2}+\left(\hat{y}_{t}^{*}\right)^{2}-\frac{2 \alpha}{(1-\alpha)(1+\phi)} \hat{y}_{t}^{*}\right] d t
$$

subject to $\left\{\hat{y}_{t}^{*}, \pi_{t}^{*}\right\}_{t \geq 0} \in \mathcal{F}$.

We know that the feasible set $\mathcal{F}$ is described by

$$
X_{t}=\alpha_{v} e^{v t} V_{v}+\frac{1}{\rho} \hat{\kappa}\left(E_{1}^{\prime} X_{0}\right) A^{-1} E_{1}
$$

where

$$
E_{i}^{\prime} X_{0}-\frac{1}{\rho} \hat{\kappa}\left(E_{1}^{\prime} X_{0}\right)\left(E_{i}^{\prime} A^{-1} E_{1}\right)=\alpha_{v} V_{v}
$$




$$
A=\left[\begin{array}{cc}
0 & -\frac{1}{(1-\alpha) \hat{\kappa}}\left(\lambda \alpha+(\hat{\kappa}(1-\alpha)+\lambda \alpha) \alpha_{\pi} \hat{\kappa}\right) \\
-\hat{\kappa} & \rho
\end{array}\right],
$$

$v$ is the negative eigenvalue of $A$ and $V_{v}$ the corresponding eigenvector.

Note that we have

$$
\operatorname{Det}(A)=-\frac{\lambda \alpha+(\hat{\kappa}(1-\alpha)+\lambda \alpha) \alpha_{\pi} \hat{\kappa}}{1-\alpha}
$$

and

$$
A^{-1}=\frac{1}{\operatorname{Det}(A)}\left[\begin{array}{cc}
\rho & \frac{\lambda \alpha+(\hat{\kappa}(1-\alpha)+\lambda \alpha) \alpha_{\pi} \hat{\kappa}}{(1-\alpha) \hat{\kappa}} \\
\hat{\kappa} & 0
\end{array}\right]
$$

so that $A^{-1} E_{1}=\frac{1}{\operatorname{Det}(A)}[\rho, \hat{\kappa}]^{\prime}=-\frac{1-\alpha}{\lambda \alpha+(\hat{\kappa}(1-\alpha)+\lambda \alpha) \alpha_{\pi} \hat{\kappa}}[\rho, \hat{\kappa}]^{\prime}$. Note in passing that this shows that a solution that starts with $E_{1}^{\prime} X_{0}>0$ ends up with $E_{1}^{\prime} X_{\infty}<0$ and $E_{2}^{\prime} X_{\infty}<0$.

We have to solve

$$
\min \frac{1}{2} \int_{0}^{\infty} e^{-\rho t}\left[X_{t}^{\prime} \Omega X_{t}-\frac{2 \alpha}{(1-\alpha)(1+\phi)} E_{1}^{\prime} X_{t}\right] d t
$$

subject to

$$
\begin{gathered}
X_{0}-\frac{1}{\rho} \hat{\kappa}\left(E_{1}^{\prime} X_{0}\right) A^{-1} E_{1}=\alpha_{v} V_{v}, \\
X_{t}=\alpha_{\nu} e^{v t} V_{v}+\frac{1}{\rho} \hat{\kappa}\left(E_{1}^{\prime} X_{0}\right) A^{-1} E_{1},
\end{gathered}
$$

where the minimization is over $X_{0}$ and $\alpha_{v}$, and

$$
\Omega=\left[\begin{array}{cc}
1 & 0 \\
0 & \alpha_{\pi}
\end{array}\right]
$$

We can use the last constraint the rewrite the objective as

$$
\begin{gathered}
\min \frac{1}{2} \int_{0}^{\infty} e^{-\rho t}\left[\left(\alpha_{v}\right)^{2} e^{2 v t} V_{v}^{\prime} \Omega V_{v}+\frac{2 \alpha_{v} \hat{\kappa}}{\rho}\left(E_{1}^{\prime} X_{0}\right) e^{v t} V_{v}^{\prime} \Omega A^{-1} E_{1}+\left(\frac{\hat{\kappa}}{\rho}\right)^{2}\left(E_{1}^{\prime} X_{0}\right)^{2} E_{1}^{\prime} A^{\prime-1} \Omega A^{-1} E_{1}\right. \\
\left.-\frac{2 \alpha \alpha_{v} e^{v t}}{(1-\alpha)(1+\phi)} E_{1}^{\prime} V_{v}-\frac{2 \alpha \frac{1}{\rho} \hat{\kappa}}{(1-\alpha)(1+\phi)}\left(E_{1}^{\prime} X_{0}\right) E_{1}^{\prime} A^{-1} E_{1}\right] d t
\end{gathered}
$$

so that we are left with the following static linear quadratic problem in three variables 
$\left(X_{0}, \alpha_{v}\right)$

$$
\begin{gathered}
\min \frac{1}{2}\left[\left(\alpha_{v}\right)^{2} \frac{1}{\rho-2 v} V_{v}^{\prime} \Omega V_{v}+\alpha_{v}\left(E_{1}^{\prime} X_{0}\right) \frac{2 \hat{\kappa}}{\rho(\rho-v)} V_{v}^{\prime} \Omega A^{-1} E_{1}+\left(E_{1}^{\prime} X_{0}\right)^{2}\left(\frac{\hat{\kappa}}{\rho}\right)^{2} \frac{1}{\rho} E_{1}^{\prime} A^{\prime-1} \Omega A^{-1} E_{1}\right. \\
\left.\quad-\alpha_{v} \frac{2 \alpha}{(1-\alpha)(1+\phi)(\rho-v)} E_{1}^{\prime} V_{v}-\left(E_{1}^{\prime} X_{0}\right) \frac{2 \alpha \frac{1}{\rho} \hat{\kappa}}{(1-\alpha)(1+\phi) \rho} E_{1}^{\prime} A^{-1} E_{1}\right]
\end{gathered}
$$

subject to

$$
X_{0}-\frac{1}{\rho} \hat{\kappa}\left(E_{1}^{\prime} X_{0}\right) A^{-1} E_{1}=\alpha_{v} V_{v} .
$$

The FOCs together with the constraints lead to a linear system of five equations in five unknowns $X_{0}, \alpha_{v}$, and the two multipliers $\lambda_{1}$ and $\lambda_{2}$ on the constraints (there are two constraints stacked in a vector). Let's write it:

$$
\begin{aligned}
& \alpha_{v} \frac{1}{\rho-2 v} V_{v}^{\prime} \Omega V_{v}+\left(E_{1}^{\prime} X_{0}\right) \frac{\hat{\kappa}}{\rho(\rho-v)} V_{v}^{\prime} \Omega A^{-1} E_{1}-\frac{\alpha}{(1-\alpha)(1+\phi)(\rho-v)} E_{1}^{\prime} V_{v}-\lambda_{1} E_{1}^{\prime} V_{v}-\lambda_{2} E_{2}^{\prime} V_{v}=0 \\
& \alpha_{v} \frac{\hat{\kappa}}{\rho(\rho-v)} V_{v}^{\prime} \Omega A^{-1} E_{1}+\left(E_{1}^{\prime} X_{0}\right)\left(\frac{\hat{\kappa}}{\rho}\right)^{2} \frac{1}{\rho} E_{1}^{\prime} A^{\prime-1} \Omega A^{-1} E_{1}-\frac{\alpha \frac{1}{\rho} \hat{\kappa}}{(1-\alpha)(1+\phi) \rho} E_{1}^{\prime} A^{-1} E_{1} \\
& +\lambda_{1}\left(1-\frac{1}{\rho} \hat{\kappa} E_{1}^{\prime} A^{-1} E_{1}\right)-\lambda_{2} \frac{1}{\rho} \hat{\kappa} E_{2}^{\prime} A^{-1} E_{1}=0, \\
& \lambda_{2}=0 \text {, } \\
& E_{i}^{\prime} X_{0}-\frac{1}{\rho} \hat{\kappa}\left(E_{1}^{\prime} X_{0}\right) E_{i}^{\prime} A^{-1} E_{1}=\alpha_{v} E_{i}^{\prime} V_{v} .
\end{aligned}
$$

Using $\lambda_{2}=0$, we can rewrite this as a linear system of four equations in four unknowns:

$$
\begin{gathered}
\alpha_{v} \frac{1}{\rho-2 v} V_{v}^{\prime} \Omega V_{v}+\left(E_{1}^{\prime} X_{0}\right) \frac{\hat{\kappa}}{\rho(\rho-v)} V_{v}^{\prime} \Omega A^{-1} E_{1}-\lambda_{1} E_{1}^{\prime} V_{v}=\frac{\alpha}{(1-\alpha)(1+\phi)(\rho-v)} E_{1}^{\prime} V_{v}, \\
\begin{array}{c}
\alpha_{v} \frac{\hat{\kappa}}{\rho(\rho-v)} V_{v}^{\prime} \Omega A^{-1} E_{1}+\left(E_{1}^{\prime} X_{0}\right)\left(\frac{\hat{\kappa}}{\rho}\right)^{2} \frac{1}{\rho} E_{1}^{\prime} A^{\prime-1} \Omega A^{-1} E_{1}+\lambda_{1}\left(1-\frac{1}{\rho} \hat{\kappa} E_{1}^{\prime} A^{-1} E_{1}\right)= \\
\frac{\alpha \frac{1}{\rho} \hat{\kappa}}{(1-\alpha)(1+\phi) \rho} E_{1}^{\prime} A^{-1} E_{1}, \\
-\alpha_{v} E_{i}^{\prime} V_{v}+E_{i}^{\prime} X_{0}-\frac{1}{\rho} \hat{\kappa}\left(E_{1}^{\prime} X_{0}\right) E_{i}^{\prime} A^{-1} E_{1}=0 .
\end{array}
\end{gathered}
$$


We can also see that $X_{0}=0$ (and $\alpha_{v}=0$ ) is not a solution since $\lambda_{1}$ cannot (generically) simultaneously solve

$$
\begin{gathered}
\lambda_{1}=-\frac{\alpha}{(1-\alpha)(1+\phi)(\rho-v)}, \\
\lambda_{1}=\frac{\alpha \frac{1}{\rho} \hat{\kappa}}{(1-\alpha)(1+\phi) \rho} \frac{E_{1}^{\prime} A^{-1} E_{1}}{1-\frac{1}{\rho} \hat{\kappa} E_{1}^{\prime} A^{-1} E_{1}},
\end{gathered}
$$

unless of course we are in the knife-edge case (but is that even possible given the signs of our variables...) where

$$
-\frac{\alpha}{(1-\alpha)(1+\phi)(\rho-v)}=\frac{\alpha \frac{1}{\rho} \hat{\kappa}}{(1-\alpha)(1+\phi) \rho} \frac{E_{1}^{\prime} A^{-1} E_{1}}{1-\frac{1}{\rho} \hat{\kappa} E_{1}^{\prime} A^{-1} E_{1}} .
$$

Since $E_{1}^{\prime} A^{-1} E_{1}=\frac{1}{\operatorname{Det}(A)} \rho=-\frac{(1-\alpha) \rho}{\lambda \alpha+(\hat{\kappa}(1-\alpha)+\lambda \alpha) \alpha_{\pi} \hat{\kappa}}$, this condition boils down to

$$
\frac{\alpha}{(1-\alpha)(1+\phi)(\rho-v)}=\frac{\alpha \hat{\kappa}}{(1-\alpha)(1+\phi) \rho} \frac{\frac{1-\alpha}{\lambda \alpha+(\hat{\kappa}(1-\alpha)+\lambda \alpha) \alpha_{\pi} \hat{\kappa}}}{1-\frac{(1-\alpha) \hat{\kappa}}{\lambda \alpha+(\hat{\kappa}(1-\alpha)+\lambda \alpha) \alpha_{\pi} \hat{\kappa}}}
$$

or

$$
\frac{\rho}{\rho-v}=\frac{\hat{\kappa}(1-\alpha)}{\lambda \alpha+\lambda \alpha \alpha_{\pi} \hat{\kappa}+\hat{\kappa}(1-\alpha)\left(\alpha_{\pi} \hat{\kappa}-1\right)}
$$

or

$$
\frac{\rho}{\rho-v}=\frac{1-\alpha}{\alpha \frac{1+\alpha_{\pi} \hat{\kappa}}{1+\phi}+(1-\alpha)\left(\alpha_{\pi} \hat{\kappa}-1\right)}
$$

\section{A.20 Coordination vs. No Coordination with Coordinated Labor Tax}

In this section we outline the coordinated labor tax case: where there is coordination on the labor tax at the symmetric steady state so that $M\left(1+\tau_{L}\right)=1$. With a slight abuse of notation, we keep denoting with a double bar the corresponding flexible price allocation with no capital controls. We denote with a double hat the gap of a variable from its flexible price counterpart. We denote with a tilde a variable minus its mean across countries. For example $\tilde{y}_{t}^{i}=\hat{y}_{t}^{i}-\hat{y}_{t}^{*}$ represents the deviation of country $i^{\prime}$ s output gap from the corresponding aggregate. 
Coordinated solution. The coordinated planning problem has the same constraint set as (21) but a different objective

$$
\frac{1}{2} \int_{0}^{\infty} \int_{0}^{1} e^{-\rho t}\left[\alpha_{\pi}\left(\tilde{\pi}_{H, t}^{i}\right)^{2}+\left(\tilde{y}_{t}^{i}\right)^{2}+(1-\alpha) \alpha_{\theta}\left(\tilde{\theta}_{t}^{i}\right)^{2}+\alpha_{\pi}\left(\pi_{t}^{*}\right)^{2}+\left(\hat{y}_{t}^{*}\right)^{2}\right] d i d t .
$$

There are two differences with the objective in (21). First, the coefficient on the term $\frac{1}{2} \hat{\theta}_{t}^{2}$ is $(1-\alpha) \alpha_{\theta}$ instead of $\alpha_{\theta}$. Second, there is no linear term in $\hat{y}_{t}^{*}$.

As above we can break down the planning problem into two parts. First, there is an aggregate planning problem determining the average output gap and inflation $\hat{y}_{t}^{*}$ and $\pi_{t}^{*}$. This aggregate planning problem has the same constraint set as (29) but a different objective

$$
\frac{1}{2} \int_{0}^{\infty} e^{-\rho t}\left[\alpha_{\pi}\left(\pi_{t}^{*}\right)^{2}+\left(\hat{y}_{t}^{*}\right)^{2}\right] d t
$$

which features no linear term in $\hat{y}_{t}^{*}$. Second, there is a disaggregated planning problem determining deviations from the aggregates for output gap, home inflation and consumption smoothing, $\tilde{y}_{t}^{i}, \tilde{\pi}_{H, t}^{i}$ and $\tilde{\theta}_{t}^{i}$. This disaggregated planning problem has the same constraint set as (30) but a different objective

$$
\frac{1}{2} \int_{0}^{\infty} \int_{0}^{1} e^{-\rho t}\left[\alpha_{\pi}\left(\tilde{\pi}_{H, t}^{i}\right)^{2}+\left(\tilde{y}_{t}^{i}\right)^{2}+(1-\alpha) \alpha_{\theta}\left(\tilde{\theta}_{t}^{i}\right)^{2}\right] d i d t,
$$

where the coefficient on $\frac{1}{2}\left(\tilde{\theta}_{t}^{i}\right)^{2}$ is $(1-\alpha) \alpha_{\theta}$ instead of $\alpha_{\theta}$. We can obtain a relaxed problem by dropping the two aggregation constraints $\int_{0}^{1} \tilde{y}_{t}^{i} d i=0$ and $\int_{0}^{1} \tilde{\pi}_{H, t}^{i} d i=0$. This relaxed planning problem can be broken down into separate component planning problems for each country $i \in[0,1]$. This component planning problem has the same constraint set as (32) but with the objective $\frac{1}{2} \int_{0}^{\infty} e^{-\rho t}\left[\alpha_{\pi}\left(\tilde{\pi}_{H, t}^{i}\right)^{2}+\left(\tilde{y}_{t}^{i}\right)^{2}+(1-\alpha) \alpha_{\theta}\left(\tilde{\theta}_{t}^{i}\right)^{2}\right] d t$.

Uncoordinated solution. With no coordination, each country $i \in[0,1]$ takes the evolution of aggregates as given and solves an uncoordinated component planning problem which has the same constraint set as (32) but a different objective

$$
\begin{aligned}
\min \frac{1}{2} \int e^{-\rho t}\left[\alpha_{\pi}\left(\tilde{\pi}_{H, t}^{i}\right)^{2}+2 \alpha_{\pi} \pi_{t}^{*} \tilde{\pi}_{H, t}^{i}+\left(\tilde{y}_{t}^{i}\right)^{2}+2 \hat{y}_{t}^{*} \tilde{y}_{t}^{i}\right. & \\
& \left.+\frac{2 \alpha}{1+\phi} \tilde{y}_{t}^{i}+(1-\alpha) \alpha_{\theta}\left(\tilde{\theta}_{t}^{i}\right)^{2} d t\right] .
\end{aligned}
$$

There are two differences with the objective in (32). First, the coefficient on $\left(\tilde{\theta}_{t}^{i}\right)^{2}$ is $(1-$ $\alpha) \alpha_{\theta}$ instead of $\alpha_{\theta}$. Second, there is a linear term in $\tilde{y}_{t}^{i}$. Intuitively, the extra linear term 
$\frac{2 \alpha}{1+\phi} \tilde{y}_{t}^{i}$ can be traced back to the fact that the that the uncoordinated solution feature terms of trade manipulation. As a result the objective acquires a preference for lower output-a form of anti-inflationary bias.

Exactly as in the case where there is no coordination on the labor tax, a central monetary authority, by setting monetary policy, can choose aggregates $\left\{\hat{y}_{t}^{*}, \pi_{t}^{*}\right\}_{t \geq 0}$ subject to the following constraints. First, it must ensure that the solutions to the uncoordinated component planning problems satisfy $\int_{0}^{1} \tilde{y}_{t}^{i} d i=0$ and $\int_{0}^{1} \tilde{\pi}_{H, t}^{i} d i=0$. This amounts to verifying a fixed point, that aggregates are actually equal to their proposed path. Second, it must ensure that the aggregate Phillips curve is verified, $\dot{\pi}_{t}^{*}=\rho \pi_{t}^{*}-\hat{\kappa} \hat{y}_{t}^{*}$. Both requirements define a set $\mathcal{F}^{\prime}$ of feasible aggregate outcomes $\left\{\hat{y}_{t}^{*}, \pi_{t}^{*}\right\}_{t \geq 0}$. Which aggregate feasible outcome is chosen depends on the objective of the central monetary authority. For example, we can examine the case where the central monetary authority seeks to maximize aggregate welfare, taking into account that capital controls are set uncooperatively. For small $\alpha$, this can be represented as a planning problem of minimizing (35) over the feasible set $\mathcal{F}^{\prime}$. 

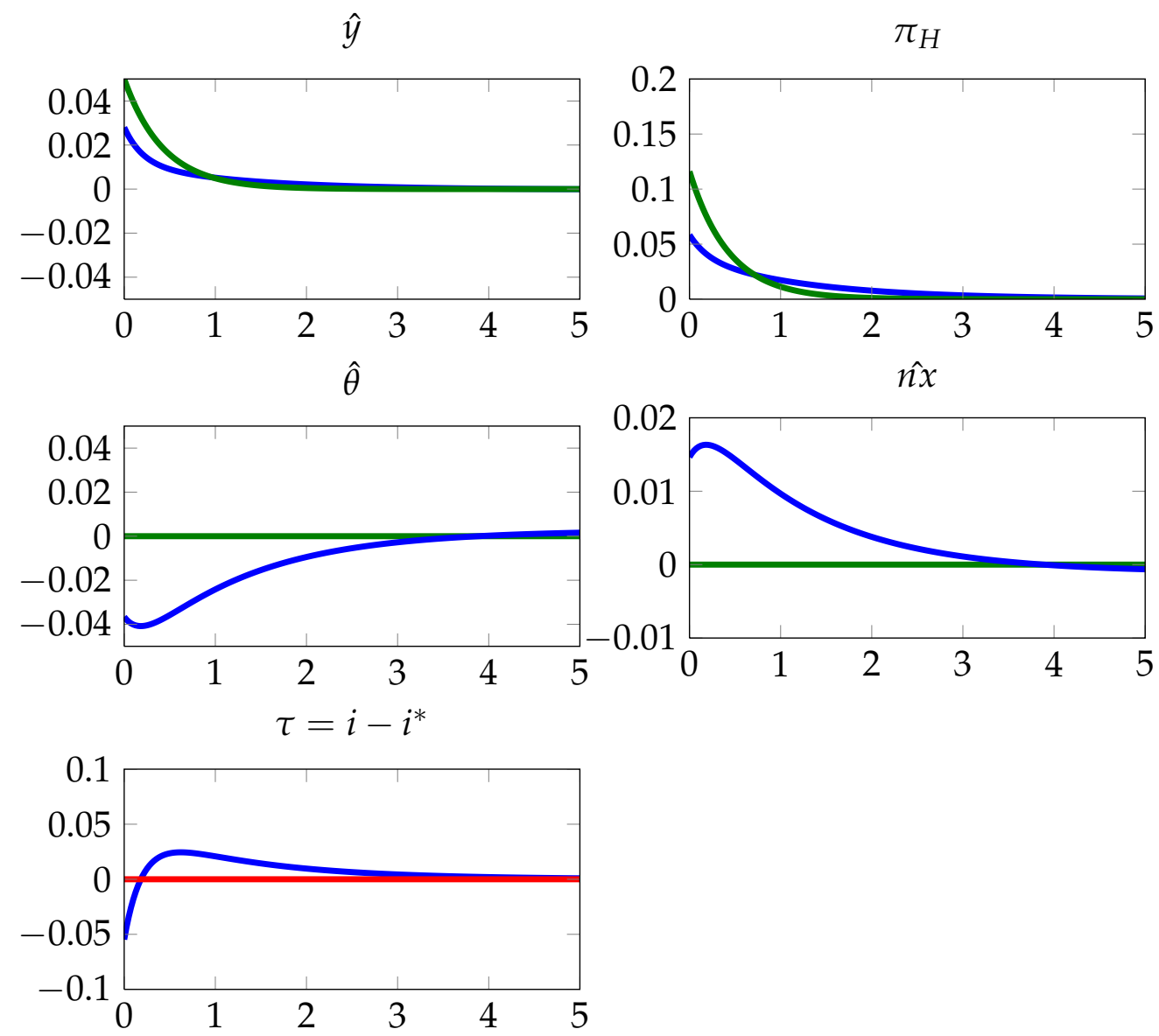

Figure 1: Permanent terms of trade shock, $\alpha=0.4$. 

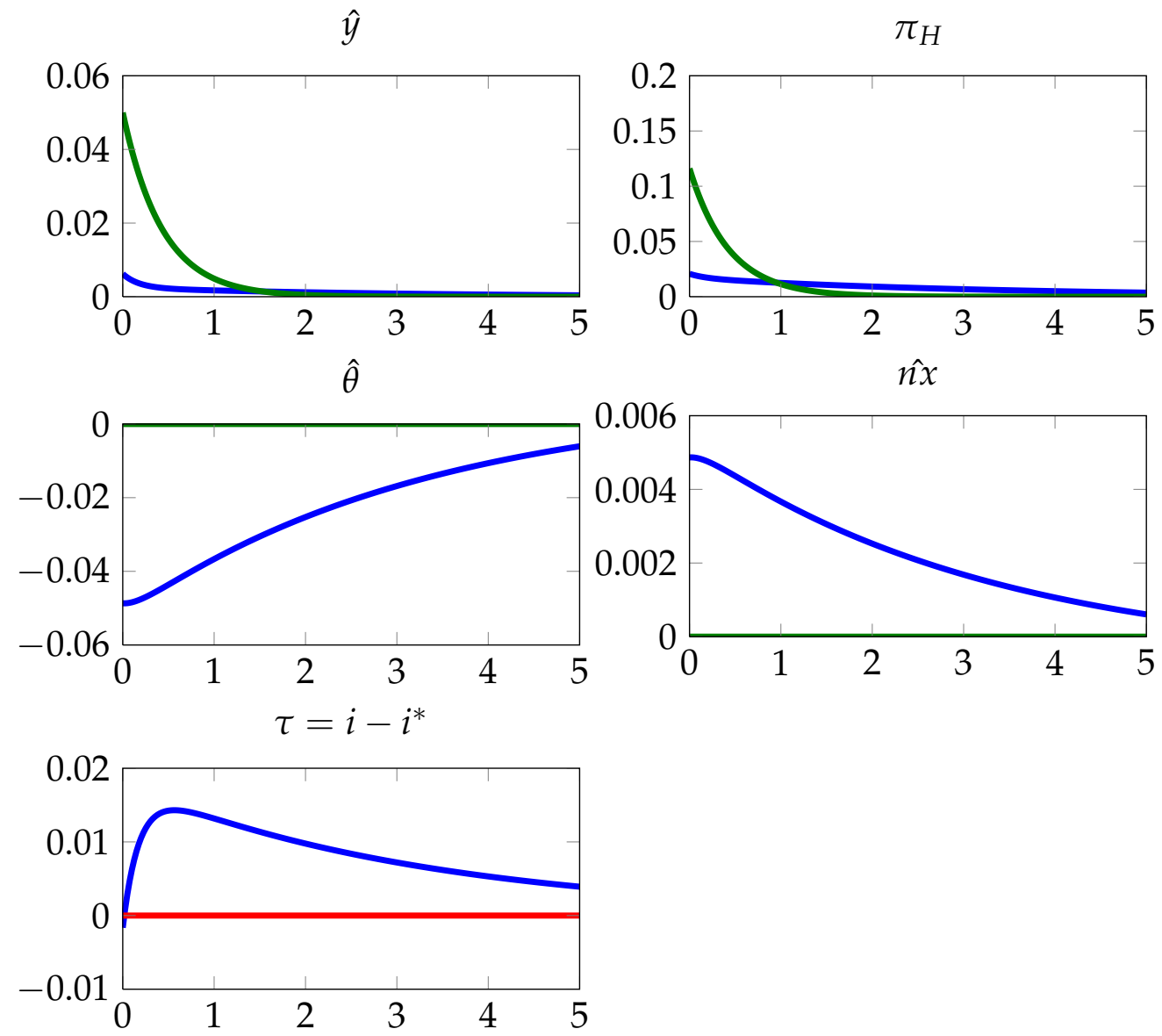

Figure 2: Permanent terms of trade shock, $\alpha=0.1$. 

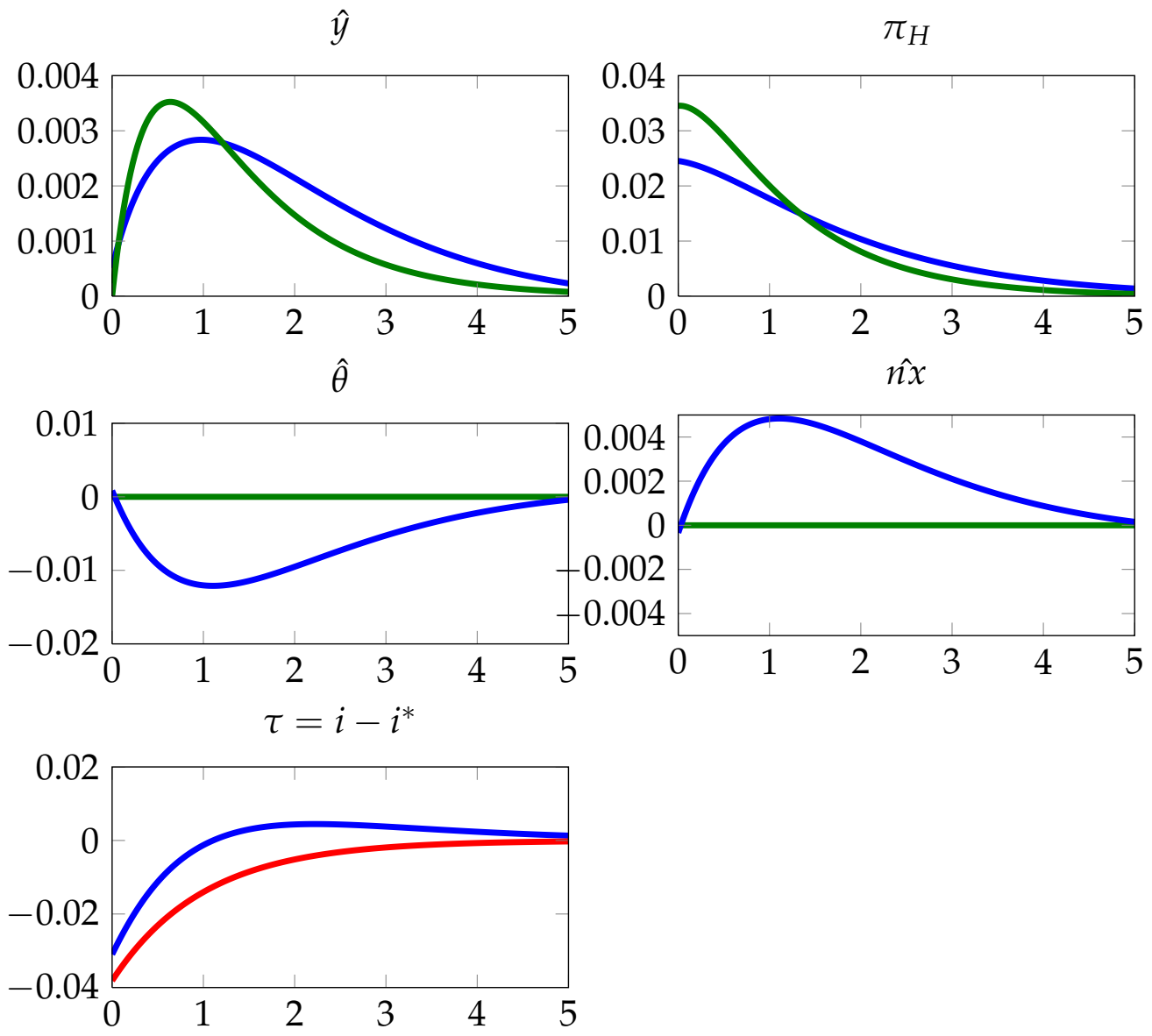

Figure 3: Mean-reverting natural interest rate shock, $\alpha=0.4$. 

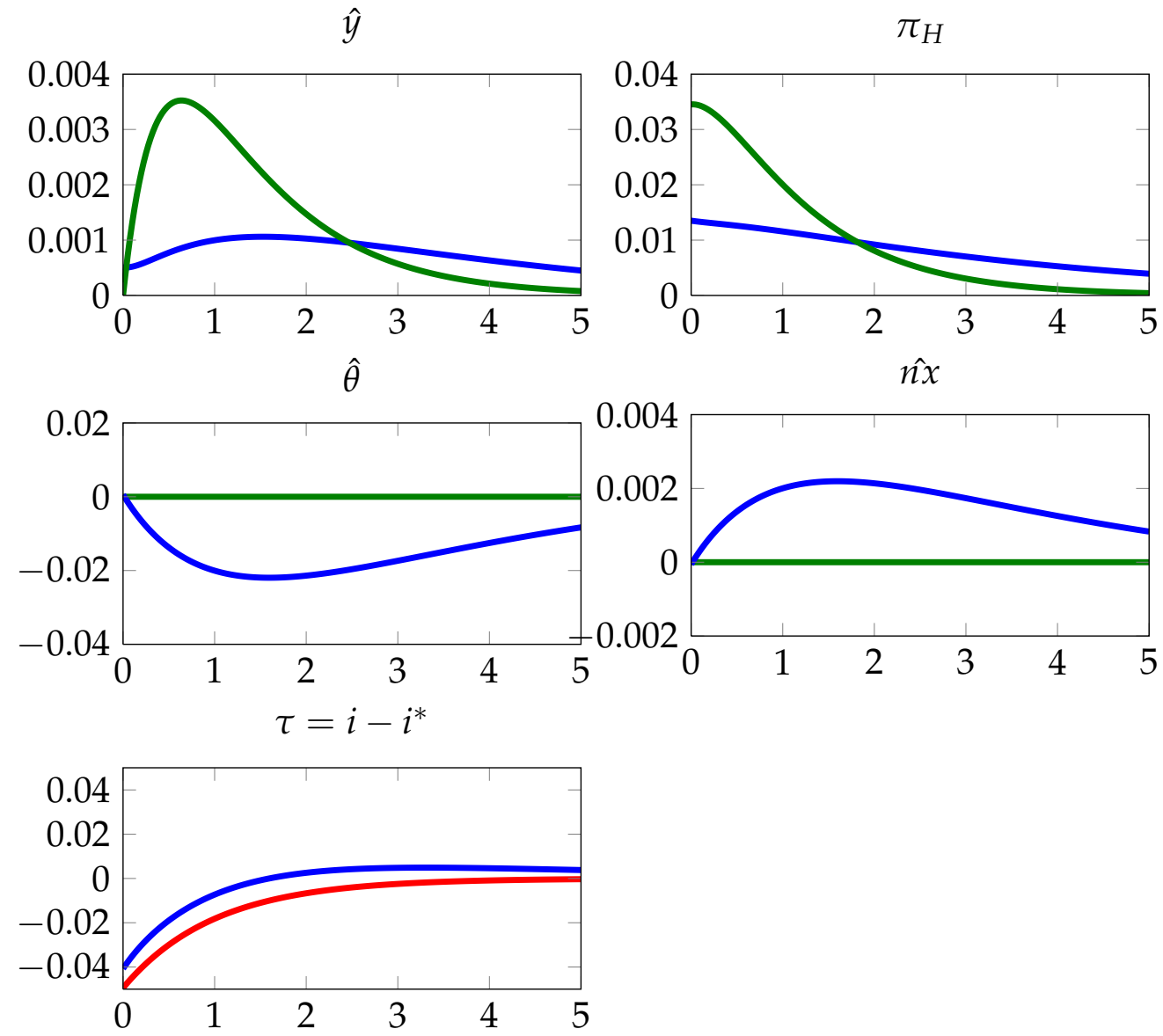

Figure 4: Mean-reverting natural interest rate shock, $\alpha=0.1$. 

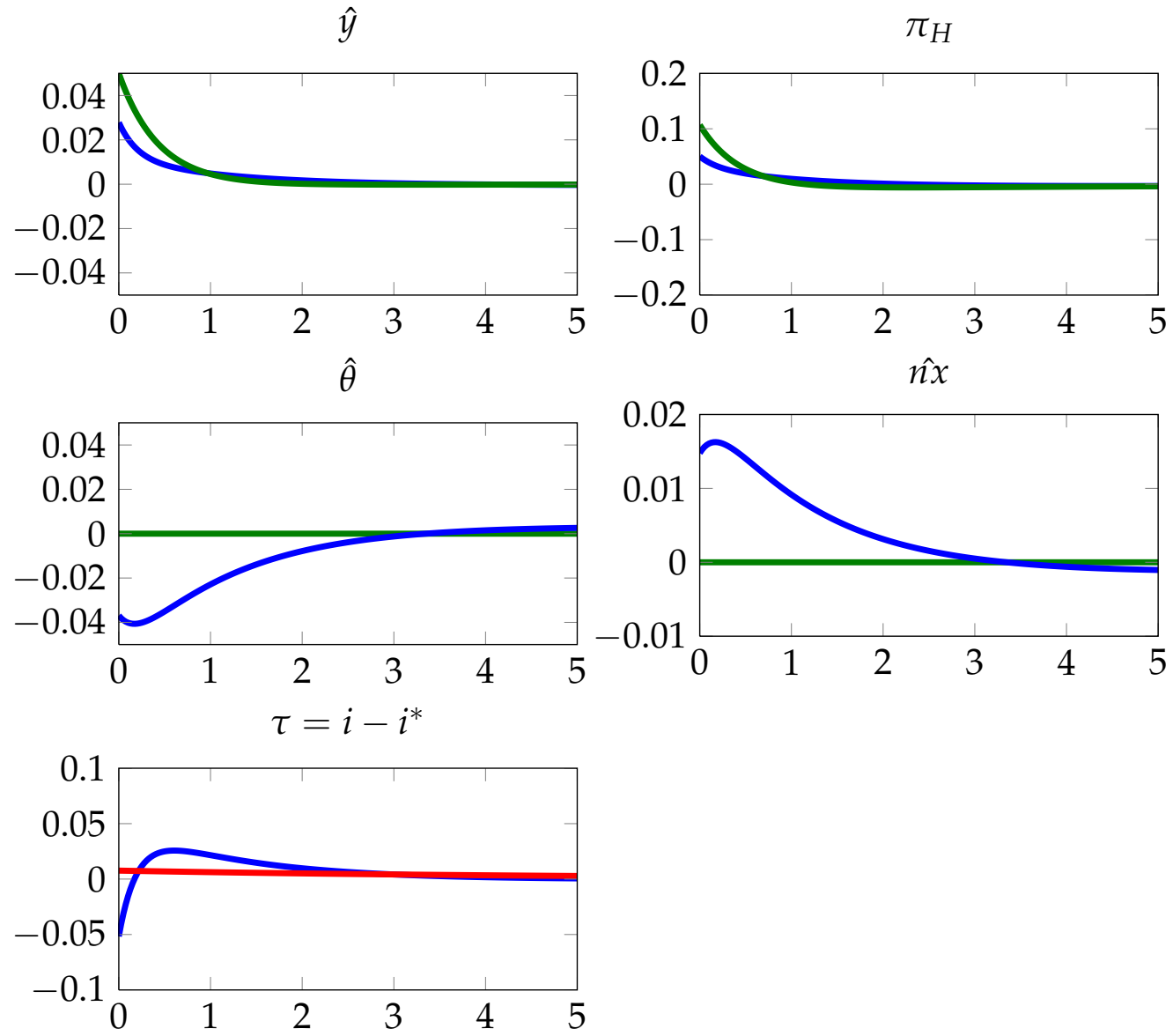

Figure 5: Mean-reverting productivity shock, $\alpha=0.4$. 

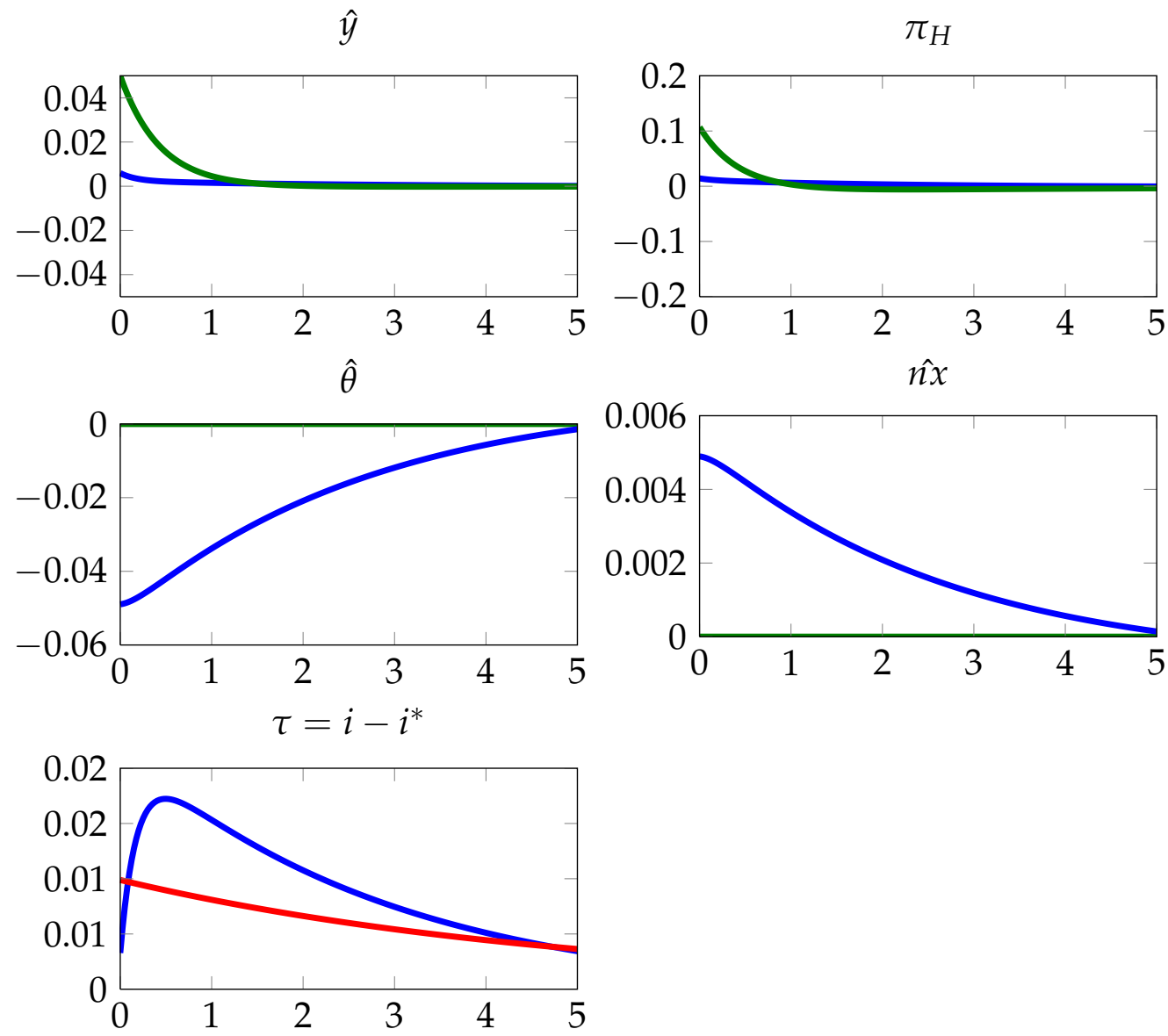

Figure 6: Mean-reverting productivity shock, $\alpha=0.1$. 

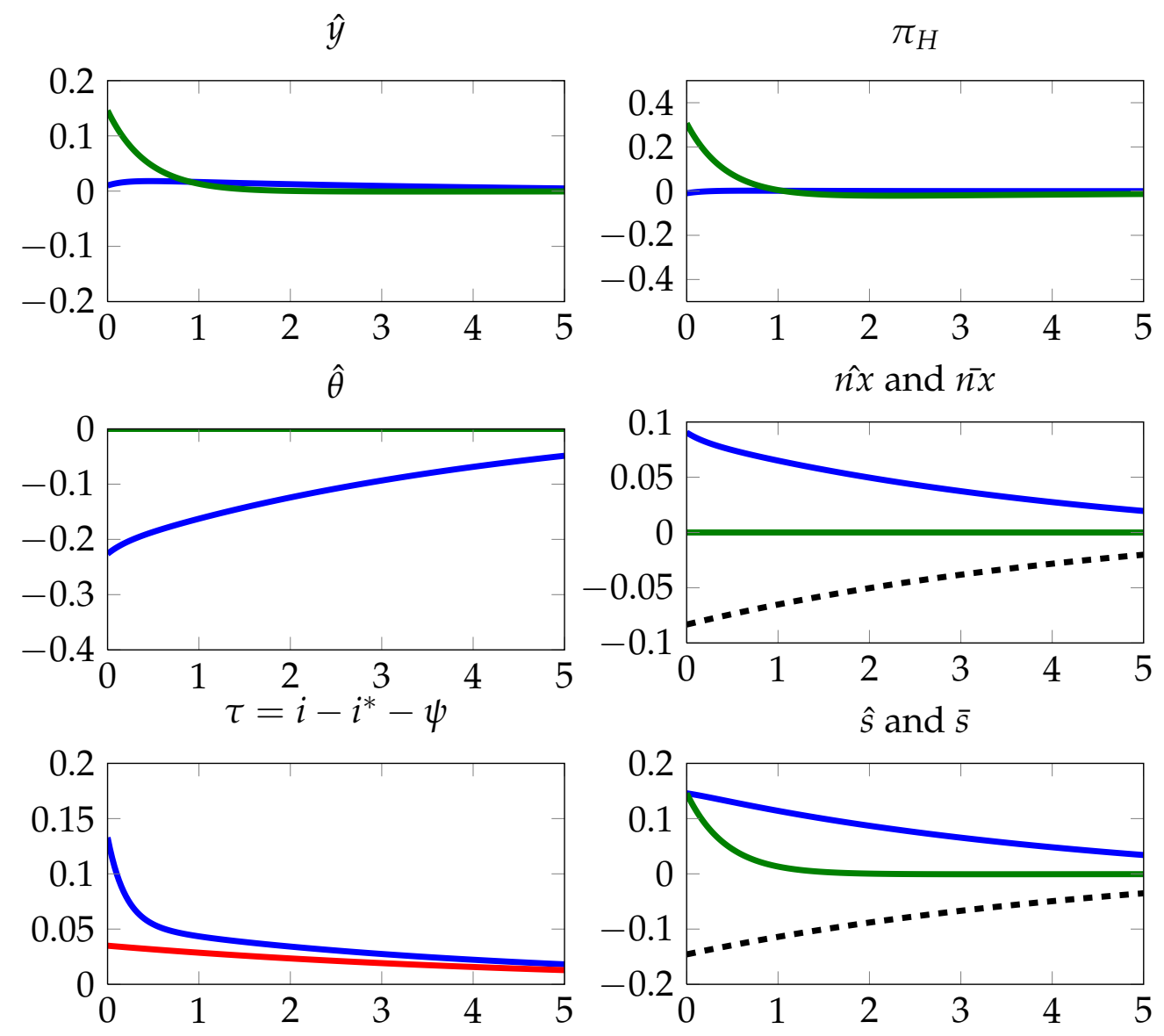

Figure 7: Mean-reverting risk premium shock, $\alpha=0.4$. 

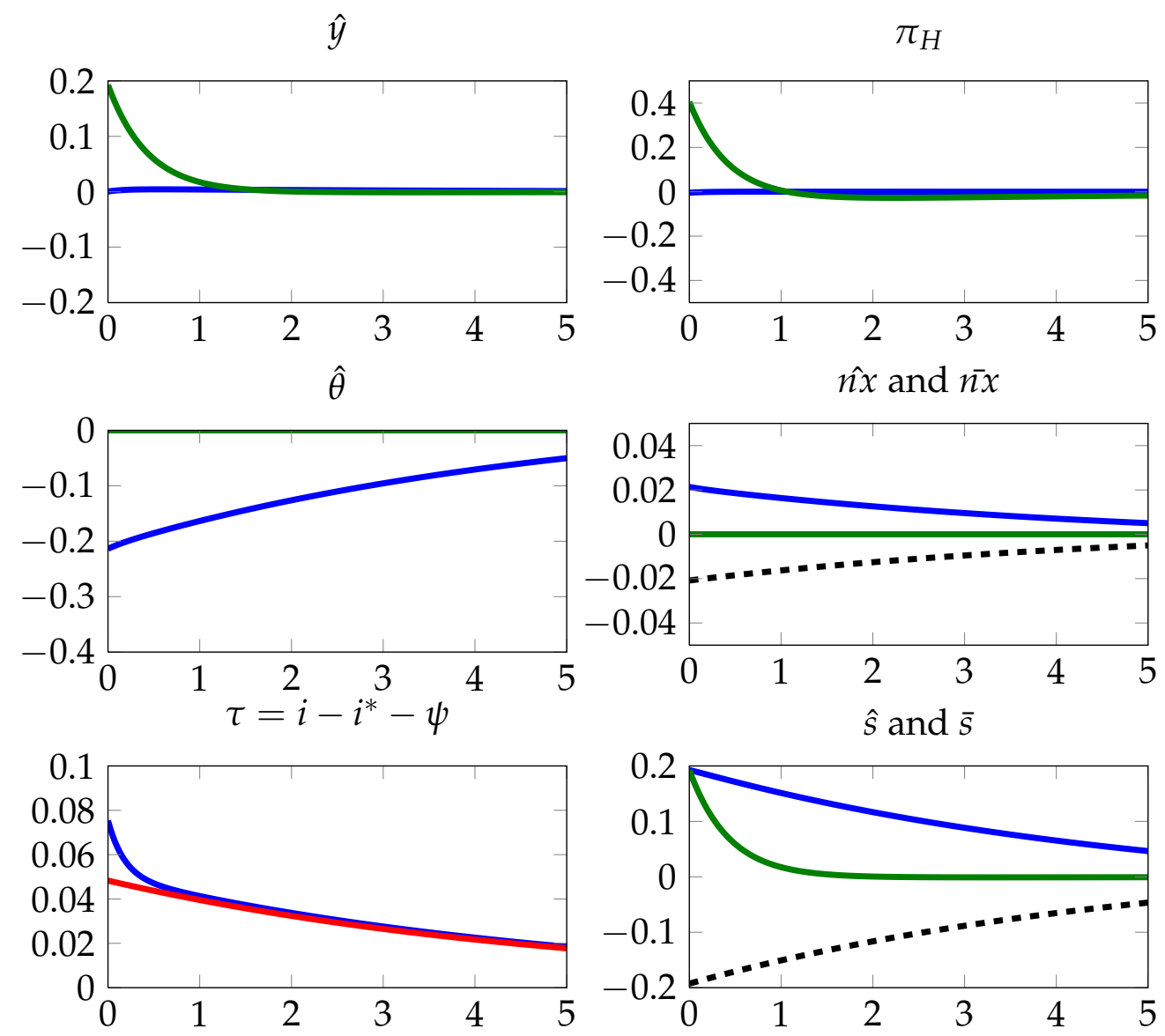

Figure 8: Mean-reverting risk premium shock, $\alpha=0.1$. 

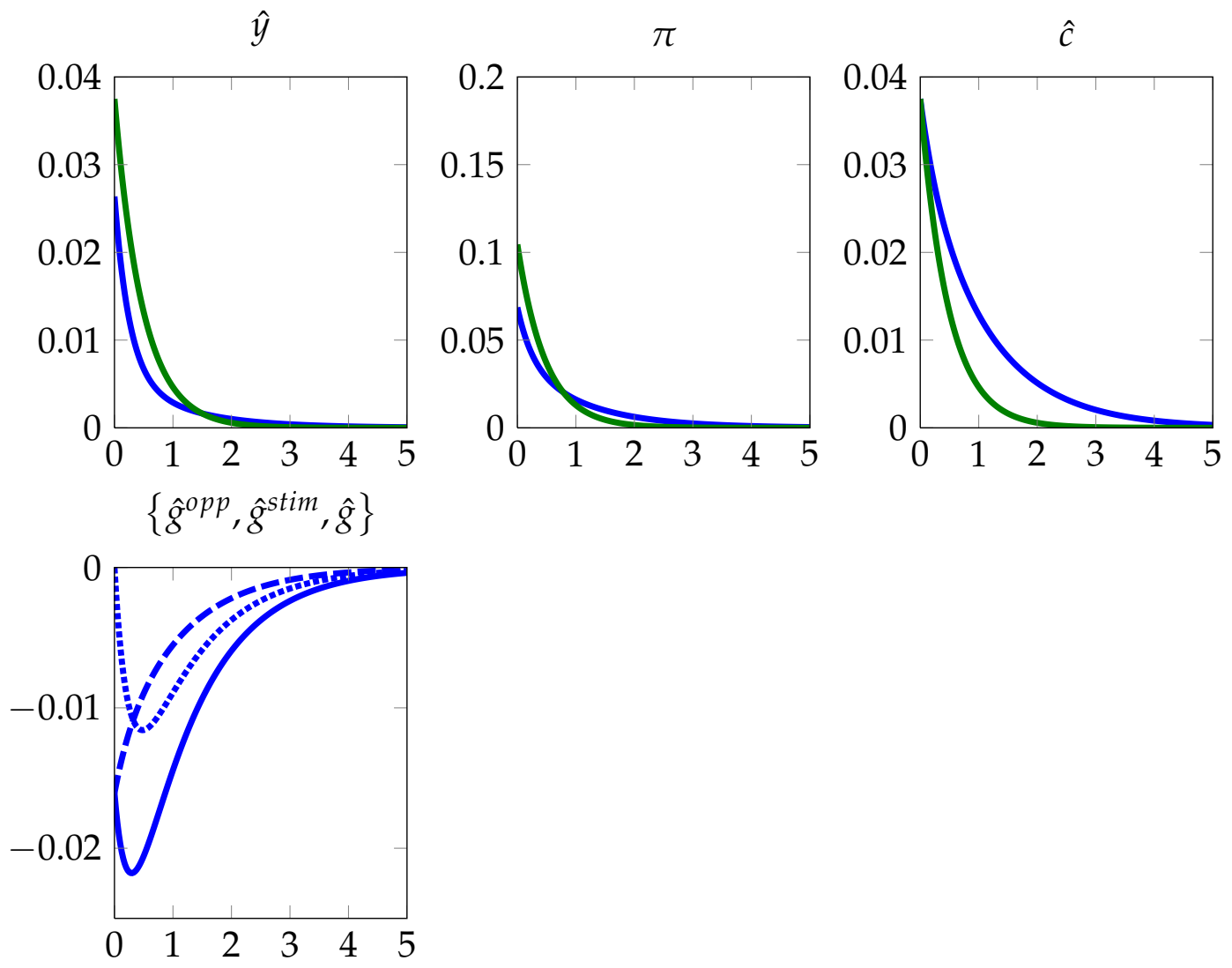

Figure 9: Government spending for a permanent terms of trade shock, $\alpha=0.4$. 

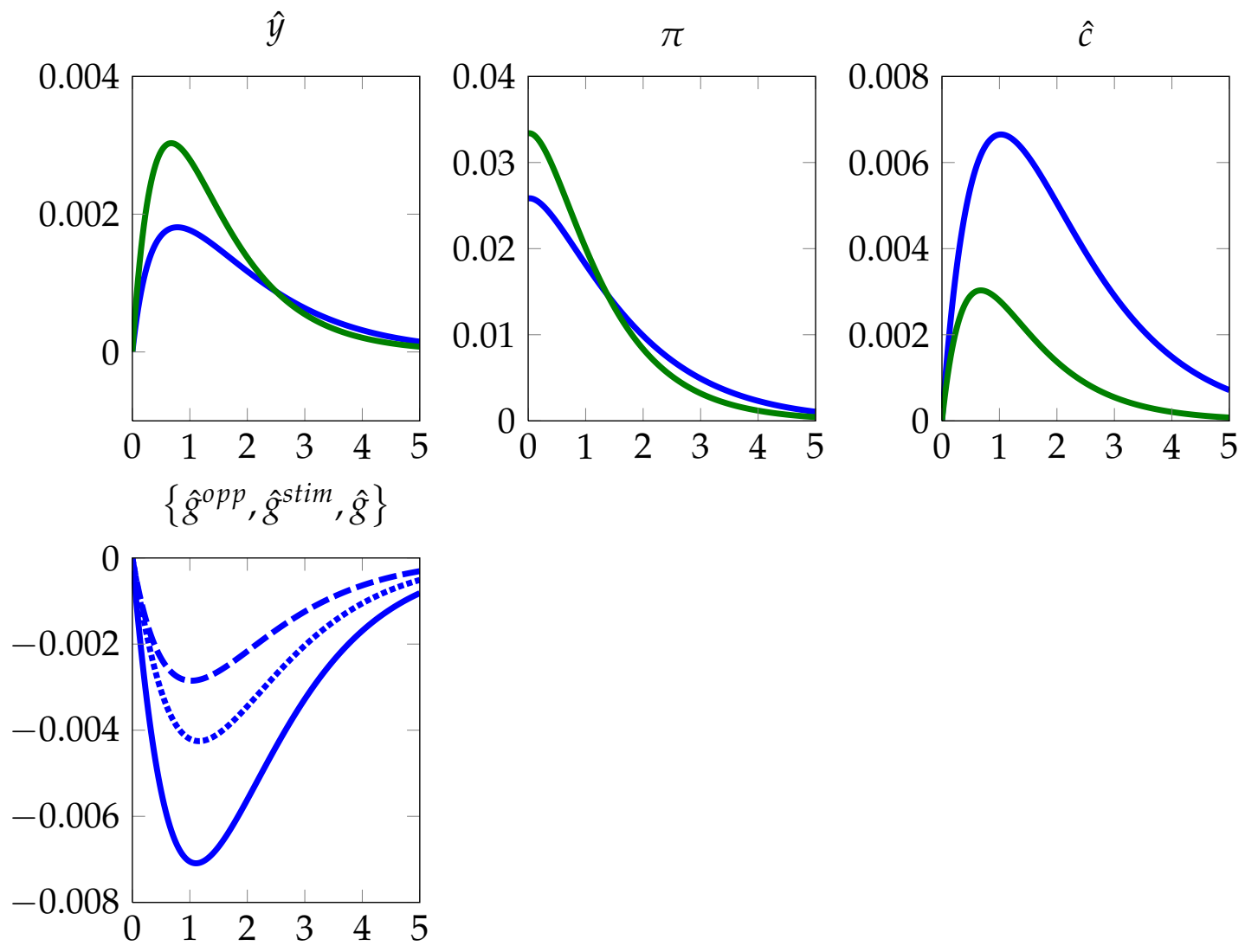

Figure 10: Government spending for a mean-reverting natural interest rate shock, $\alpha=0.4$. 


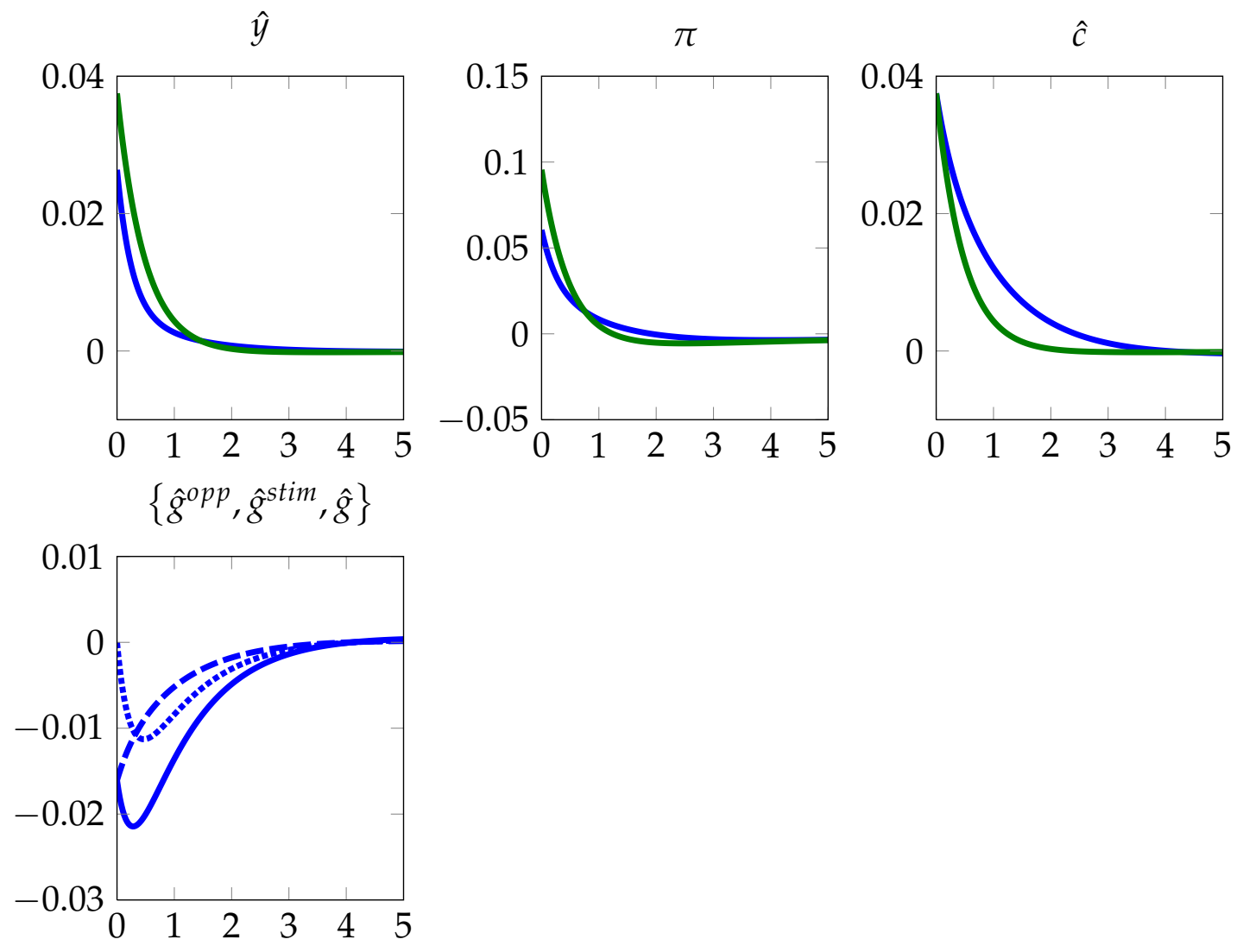

Figure 11: Government spending for a mean-reverting productivity shock, $\alpha=0.4$. 